Universitat Politècnica de València

Departamento de Química

\title{
Combination of nanophotonic biosensors and light-assisted immobilization procedures for the detection of cardiac biomarkers
}

\author{
Jad Sabek \\ Supervisors: Dr. Jaime García Rupérez \\ Dr. María José Bañuls Polo
}

Thesis submitted in partial fulfilment of the requirements for the degree of Doctor of Philosophy by the Universitat Politècnica de València. 



\section{Acknowledgments}

It is a high cause of gratitude to acknowledge those who have helped me personally and professionally and contributed to make possible this $\mathrm{PhD}$ Thesis.

I would like to acknowledge first of all my supervisors Jaime García Rupérez and María José Bañuls Polo. You gave me the opportunity to develop these three years of $\mathrm{PhD}$ Thesis on the Experimental Techniques in Chemistry program at the Nanophotonics Technology Center (NTC) of the Universitat Politècnica de València. You had patience and expertise to led me to bring all my best to reach this goal. Thank you honestly for everything.

Warm thanks to all the NTC team who helped me to develop my work. Thank you, Laurent, Todora, Miroslavna, Cristina, David, Luis, Elena and Amadeu for your high availability whenever I need it.

To my Biophotonics group colleagues, Raffaele, Luis, Xisco, David, Angela, Maribel and specially Paula, are highly acknowledged for their high support, professional and personal. Many thanks to all of you.

Thanks also to the SYM colleagues, Rafael, Dani and Zeneida for their implication, hardworking and collaboration to take ahead this project.

At the NTC I have met many people with very high professional and human qualities, and it was a honor for me having such kind of people as friends. Kike, Inma, Angie, Giuseppe, Javi, Fran and Luis. You really did my day by day pleasant. Always when I needed to talk you were always present. My Valencia's family. Really thank you very much. You know perfectly how much I love you friends.

Cannot forget to thank my Tarragona's family. Very thankful to Tsegai Medine, Ciara O’Sullivan, F. X. Rius, Jordi Riu, Patri Llorens, Santiago Macho, Tomás Guinovart, 
Marc Parrilla, Marta Novell, Soufiane, Amjed, Tesfa, Fete, Wonde and James Muye (RIP). We will never forget you.

My deepest gratitude to all my family, especially to my Mum, Naima Khalifa, my dad Bouchaïb Sabek, my brother Yassine Sabek and my lovely sister Doha Sabek. 


\section{Abstract}

Healthcare is a field where the early detection of diseases is becoming more and more important. Nowadays, professionals and citizens demand high quality diagnosis techniques offered by both private and public health systems. When the application of diagnostic tests is not adequate, different consequences can be observed such as health hazard and technical and economic overload of health services. This is due to the fact that the diagnostic techniques available are expensive, centralized in laboratories and with the need for highly qualified professionals to carry out these tasks, what can fundamentally lead to delays in time, being critical for the patient's health. It is very necessary, therefore, to reflect on the need and emergency of such preventive practices, especially for highrisk diseases such as cancer, Alzheimer or the first cause of death in the world, the cardiovascular diseases.

Within this context, the main objective of the work done during this $\mathrm{PhD}$ Thesis is to help on overcoming these problems by exploring the possibility of using photonic technology for the development of analysis devices which might be used for the early diagnosis and prognosis of cardiovascular diseases. This objective has been addressed by combining nanophotonic technology, by the nanofabrication of the photonic PBG sensing structures, which provides several benefits such as a high sensitivity, an extreme size reduction and a fabrication process being compatible with that from the microelectronics industry, with a light-assisted biofunctionalization method forming a stable and selective biorecognition layer using TEC reaction able to provide extremely thin biorecognition layers with a spatially-selective immobilization. 


\section{Resumen}

El cuidado de la salud es un campo en el que la detección precoz de enfermedades está cobrando cada vez más importancia. Hoy en día, profesionales y ciudadanos demandan que las técnicas de diagnóstico sean de alta calidad, tanto para el sistema de sanidad privado como para el público. Cuando se utilizan técnicas de diagnóstico de manera inadecuada, eso puede acarrear bastantes consecuencias, tales como un serio peligro sobre la salud y la sobrecarga técnica y económica de los servicios de salud. Eso es debido a que las técnicas de diagnóstico disponibles hoy en día son demasiado costosas, centralizadas en laboratorios y necesitan profesionales altamente cualificados para poder llevar a cabo dichas tareas, lo que conllevaría una demora en el tiempo, siendo este muchas veces vital para los enfermos. Es muy necesario, por lo tanto, reflexionar sobre la necesidad y emergencia de tales prácticas preventivas, especialmente para enfermedades de alto riesgo como el cáncer, el Alzheimer o la primera causa de muerte en el mundo, las enfermedades cardiovasculares.

En este contexto, el objetivo principal del trabajo realizado durante esta Tesis Doctoral es ayudar a superar estos problemas mediante la exploración de la posibilidad de utilizar tecnología fotónica para el desarrollo de sistemas de análisis que puedan ser utilizados para el diagnóstico y pronóstico de las enfermedades cardiovasculares. Este objetivo se ha abordado mediante la combinación de la tecnología nanofotónica, consistiendo en la nanofabricación de las estructuras PBG de sensado que ofrece varios beneficios, como una alta sensibilidad, una extrema reducción de tamaño y un proceso de fabricación compatible con el de la industria microelectrónica, con un método de biofuncionalización obteniendo una capa de bioreconocimiento estable y selectiva mediante el uso de la reacción TEC asistida por luz capaz de proporcionar unas capas de bio-reconocimiento extremadamente finas con una inmovilización espacialmente selectiva. 


\section{Resum}

L'atenció a la salut és un camp en què la detecció precoç de malalties està cobrant cada vegada més importància. Hui en dia, professionals i ciutadans demanen que les tècniques de diagnòstic siguin d'alta qualitat, tant per al sistema de sanitat privat com per al públic. Quan s'utilitzen tècniques de diagnòstic de manera inadequada, això pot comportar bastants conseqüències, com ara, un seriós perill sobre la salut i la sobrecàrrega tècnica i econòmica dels serveis de salut. Això és degut al fet que les tècniques de diagnòstic disponibles hui en dia són molt costoses, centralitzades en laboratoris i necessiten professionals altament qualificats per poder realitzar aquestes tasques, lo que comportaria a una demora en el temps que moltes vegades es vital pels malalts. És molt necessari, per tant, reflexionar sobre la necessitat i emergència de tals pràctiques preventives, especialment per a malalties d'alt risc com el càncer, l'Alzheimer o la primera causa de mort al món, les malalties cardiovasculars.

En aquest context, l'objectiu principal del treball realitzat durant aquesta Tesi Doctoral és ajudar a superar aquests problemes mitjançant l'exploració de la possibilitat d'utilitzar tecnologia fotònica per al desenvolupament de sistemes d'anàlisis que puguin ser utilitzats per al diagnòstic i pronòstic de les malalties cardiovasculars. Aquest objectiu s'ha abordat mitjançant la combinació de la tecnologia nanofotònica, consistint en la nanofabricació de les estructures de detecció de PBG fotòniques que ofereix diversos beneficis, com una alta sensibilitat, una extrema reducció de mida i un procés de fabricació compatible amb el de la indústria microelectrònica, amb un mètode de biofuncionalització obtenint una capa de bio-reconeixement estable i selectiva mitjançant l'ús de la reacció TEC assistida per llum capaç de proporcionar unes capes de bioreconeixement extremadament fines amb una immobilització espacialment selectiva. 


\section{Abbreviations}

ADC: analog-to-digital converter.

AFM: Atomic Force Microscope.

AntiBSA: BSA antibody.

APTES: (3-Aminopropyl)triethoxysilane.

BSA: Bovine Serum Albumin.

CCD Camera: Charged Coupled Device Camera.

CRP: C-Reactive Protein.

cTn: Cardiac Troponin.

cTnC: cardiac Troponin Calcium.

cTnI: cardiac Troponin Inhibitor.

cTnT: cardiac Troponin Tropomyosin.

CVD: Cardiovascular diseases.

DNA: Deoxyribonucleic acid.

DIW: Deionized Water. 
EBL: Electron Beam Lithography.

EDTA: Ethylenediaminetetraacetic acid.

EHN: European Heart Network.

ELISA: Enzyme-Linked ImmunoSorbent Assay.

EtOH: Ethanol.

FDTD: Finite-difference time-domain method.

Gln: Glutamine.

Glu: Glutamic acid.

$\mathrm{H}_{2} \mathrm{O}_{2}$ : Hydrogen peroxide.

$\mathrm{H}_{2} \mathrm{SO}_{4}$ : Sulfuric acid.

H-Bonds: Hydrogen bonds.

hIgG: Half immunoglobulin G.

HSQ: Hydrogen Silsesquioxane.

IgG: Immunoglobulin G.

IR: Infrared.

IRRAS: Infrared Resonance Absorbance Spectroscopy.

$\mathrm{KCl}$ : Potassium chloride.

KOH: Potassium hydroxide.

Leu: Leucine.

LOC: Lab On a Chip.

LOD: Limit Of Detection.

Lys: Lysine.

Mb: Myoglobin.

MPB: MIT Photonic Bands. 
$\mathrm{NaCl}$ : Sodium chloride.

$\mathrm{NaOH}$ : Sodium hydroxide.

PBG: Photonic Bandgap.

PBS: Phosphate-Buffered Saline.

PBS-T: Phosphate-Buffered Saline-Tween20.

PCR: Polymerase Chain Reaction.

PDB: Protein Data Bank.

PDMS: Polydimethylsiloxane.

PMMA: polymethyl methacrylate.

POCT: Point Of Care Testing.

RCSB: Research Collaboratory for Structural Bioinformatics.

RI: Refraction Index.

S: Sensitivity.

SEM: Scanning Electron Microscope.

Si: Silicon.

$\mathrm{SiO}_{2}$ : Silicon dioxide.

SNOM: Scanning Near-field Optical Microscope.

SOI: Silicon On Insulator.

SU-8: Sukhoy-8.

TCEP: tris(2-Carboxyethyl)phosphine.

TEC: Thiol-ene Chemistry.

TEVS: Triethoxy-vinyl sialne.

UV: Ultraviolet.

WCA: Water Contact Angle. 


\section{Index}

Acknowledgements...........................................................................................................I

Abstract...................................................................................................................III

Resumen.......................................................................................................................

Resum.............................................................................................................................VI

Abbreviations...........................................................................................

\section{Introduction}

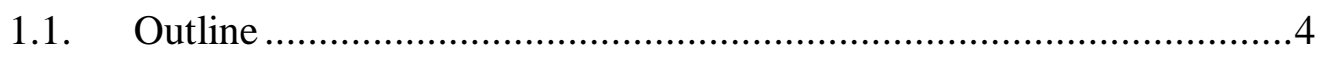

1.2. Cardiovascular diseases (CVD) biomarkers.....................................

1.3. Evanescent wave-based biosensors....................................................14

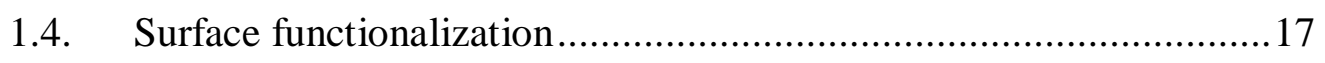

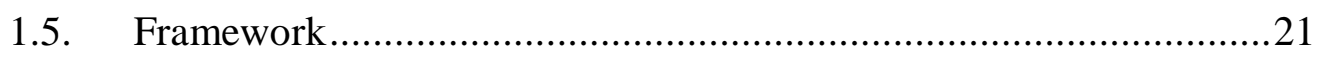

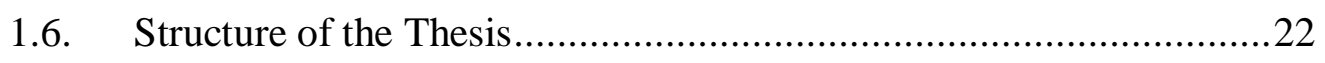

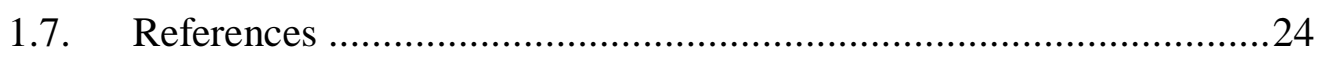

2. Computational study of the cardiac Troponin I interaction

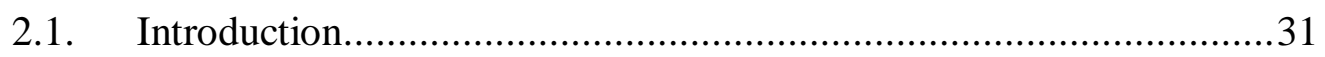

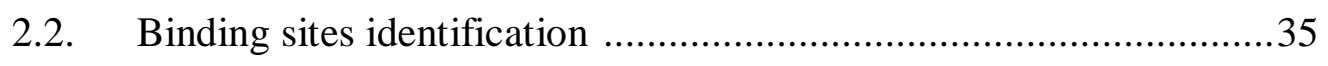

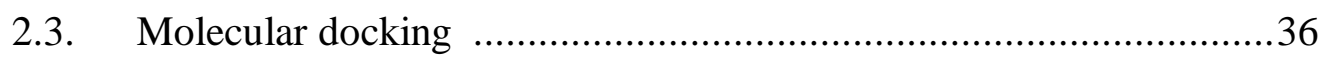

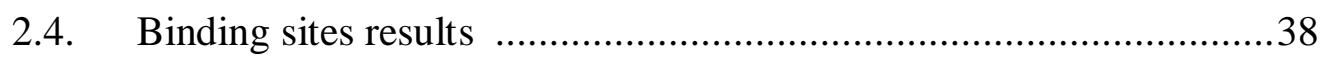

2.5. Molecular docking results ………………………..........................

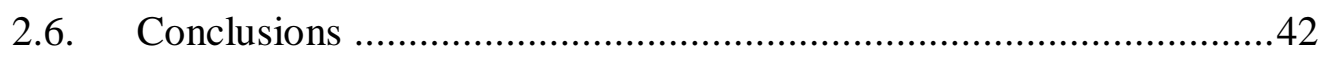




\section{Real-time tracking of the biofunctionalization by PBG biosensors}

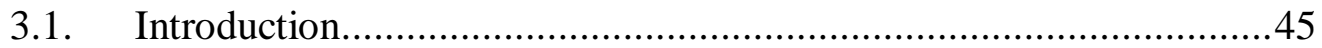

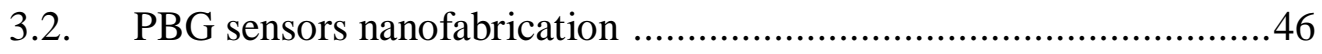

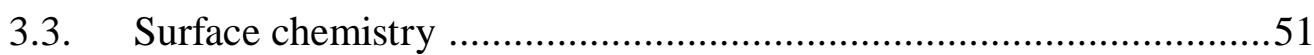

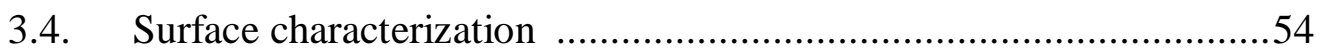

3.5. UV Light assisted immobilization of the half antibodies..................55

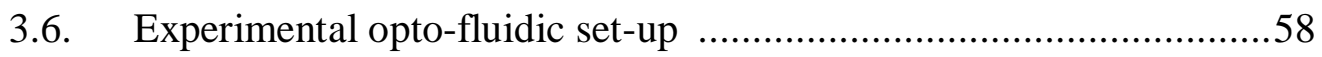

3.7. Real-time monitoring of the half $\mathrm{IgG}$ immobilization .....................62

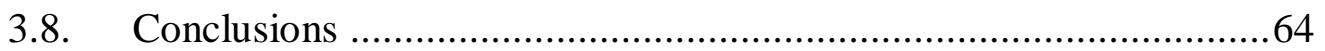

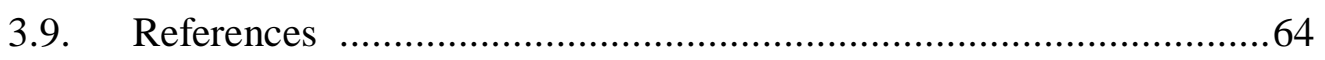

4. BSA protein direct detection by PBG biosensors

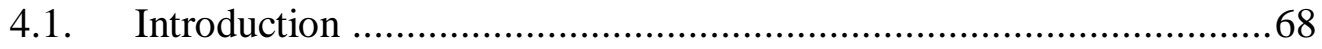

4.2. Scanning Near Field Optical Microscope (SNOM) characterization .69

4.3. Drop-casting half antibody biofunctionalization ........................... 74

4.4. Direct and real-time BSA protein detection by PBG biosensors........75

4.5. Direct and real-time detection of CVD biomarkers ........................78

4.5.1. C-Reactive protein (CRP) detection...............................79

4.5.2. Myoglobin (Mb) detection........................................... 80

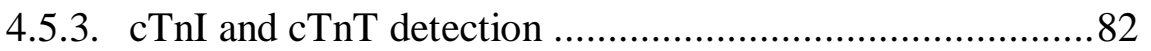

4.6. Alternatives to half antibody and $1 \mathrm{ng} / \mathrm{mL} \mathrm{cTnT}$ detection..................84

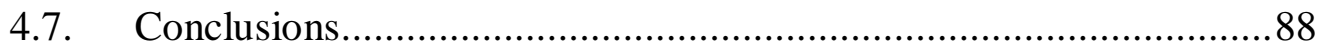

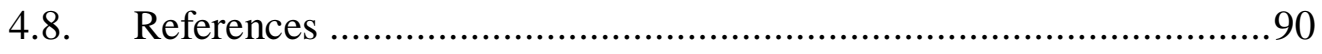


5. Conclusions and future steps

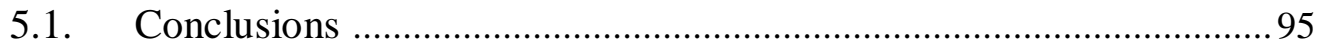

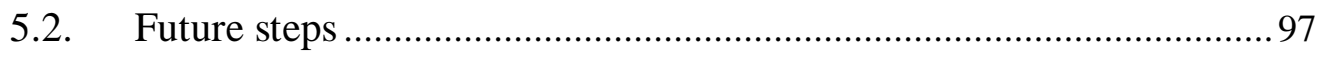

\section{Author publications}

Journal publications ..................................................................................98

Conference publications ..................................................................................99 


\section{Chapter 1}

\section{Introduction}

\subsection{Outline}

Nowadays, point of care testing (POCT) market is perceiving an even increasing demand for novel, effective and cheap devices for diseases early diagnosis/detection. Among these diseases, there is an even special interest for cardiovascular diseases (CVD) which is the leading cause of death worldwide with 17.9 million deaths. The recent statistical report of 2017 of the European Heart Network (EHN) depicts that CVD are the leading cause of death in Europe with 3.9 million death per year (40\% in the European Union). Data according to the European Heart Network (EHN, 2017) are shown in figure 1.1, with an estimated cost of about $210 €$ Billion per year. A total amount of $111 €$ Billions is related with healthcare costs, especially diagnosis and drug therapies, while the rest is for nonhealthcare dispenses. Despite these numbers, CVD can be successfully treated once detected correctly and prematurely. From here, the high interest of the implementation of effective detection tools, since they are extremely interesting for reducing mortality rates. 


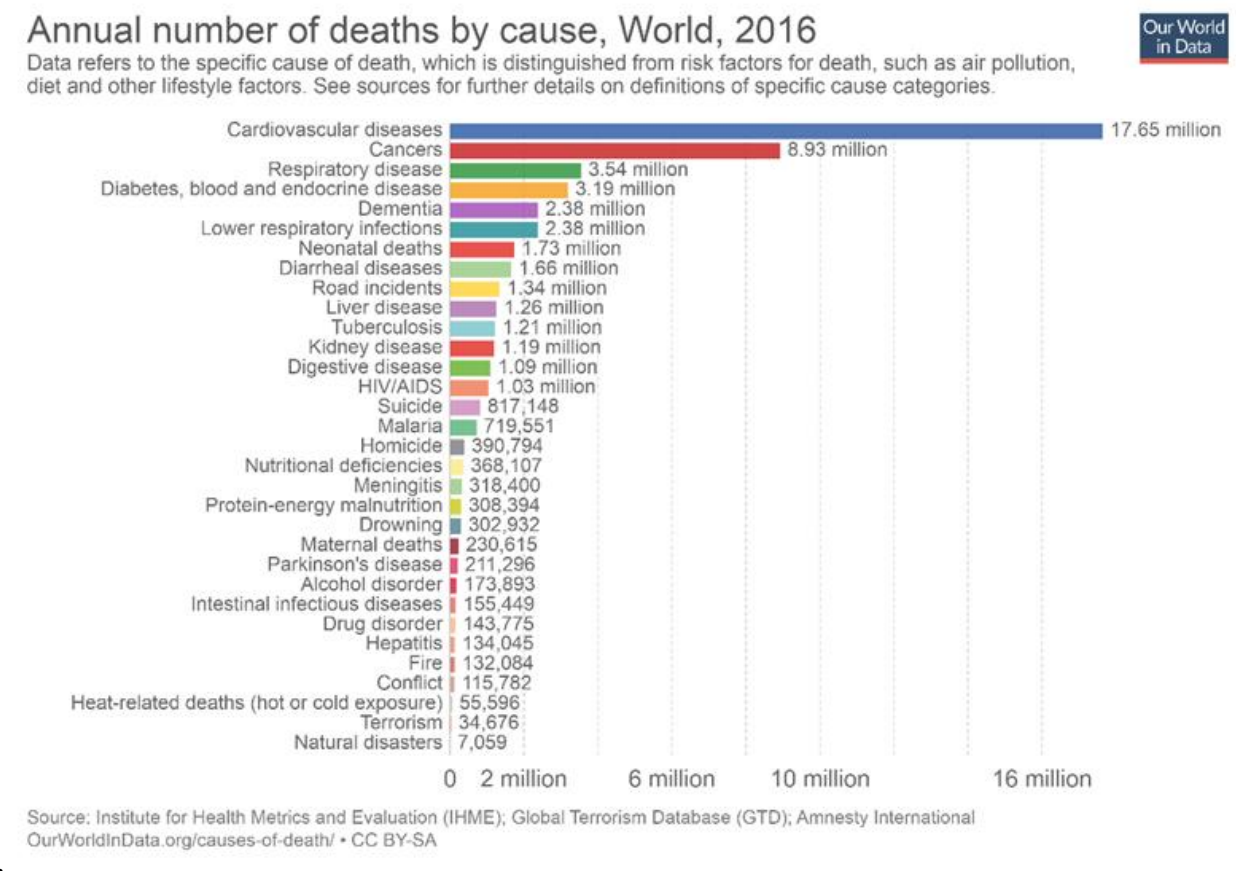

Figure 1.1. Worldwide deaths annual number by causes. Source: European Heart Network organization report (2017).

In cardiovascular diseases, heart failure is the end stage (Pagidipati and Gaziano, 2013). That heart failure is commonly produced by a damage of the heart myofilaments that can lead to systolic failure, resulting on a decrease of the blood circulation, not sufficing organism needs (Lee Goldman et al., n.d.). Specifically, we can establish that in the correct operation of the cardiovascular system, influences not only blood and heart but also the corresponding blood vessels and the lymphatic system. This is the last step for the correct working of the transport system, both for the regulation of liquids and for defenses and fatty acids. In the case of blood vessels, they are classified into elastic arteries, arterioles, muscle arteries, capillaries and veins (Hurst et al., 1974).

\section{Types of cardiovascular diseases:}

- Coronary heart disease (CHD) is the most common type of heart disease and occurs when plaque builds up in the arteries that lead to the heart. It is also called coronary artery disease (CAD). When the arteries narrow, the heart cannot receive enough blood and oxygen and can cause a heart attack. Over 
time, CHD can weaken the myocardium and cause heart failure or arrhythmias.

- Heart failure occurs when the myocardium becomes stiff or weak and it cannot pump enough oxygenated blood, which causes symptoms throughout the body. The disease can affect only the right side or the left side of the heart. Nevertheless, it is very common that both sides of the heart are compromised. High blood pressure and CAD are common causes of heart failure.

- Arrhythmias are problems with heart rate (pulse). This happens when the electrical system of the heart does not work properly, making it to beat too fast, too slow or irregularly. Some heart problems, such as a heart attack or heart failure, can cause problems with electrical system of the heart. Some people are born with an arrhythmia.

- A stroke is caused by a lack of blood flow to the brain. This can happen due to a blood clot that travels to the blood vessels in the brain or a bleeding in the brain. Stroke has many of the same risk factors as heart disease.

- Congenital heart disease is a problem with the structure and functioning of the heart that is present at birth. This term can describe many different problems that affect the heart. It is the most common type of congenital anomaly.

As for almost all diseases, an early detection of CVD is crucial for an efficient treatment and prognosis. Thus, the development of an effective, sensitive and reliable detection tool is of a high importance. In this sense, biosensors should meet all of the requirement needed for such detection devices.

A biosensor is an analytical device used in the detection of chemical and/or biological substances, which combines chemical and biological components with a transducer. The different components of a biosensor are:

- Recognition layer: Known as the surface or interface of interaction between the receptor and the target analyte/molecule. Chemical receptors, 
nucleic acids, aptamers, enzymes and antibodies are some examples of these recognition elements.

- Transducer: It is the element responsible for converting the biorecognition event taking place in the recognition layer into a reliable and measurable output physical signal. Electrochemical, optical and mechanical transducers are some examples of transducer.

- Signal processor: It is in charge of acquiring and processing the signal from the transducer.

Among other parameters, sensitivity, selectivity, response time and limit of detection are the characteristic parameters to evaluate the biosensing device performance:

- Sensitivity: is the relationship between the variation of the sensor output signal as a response to a physical quantity variation and the physical quantity variation itself. In general, sensitivity is the slope of the calibration curve. This parameter defines the ability of the sensor to detect that variation and to transduce it into a measurable signal.

- Selectivity: refers to the capability of the sensor to detect the specific target analyte with no interference with other non-targeted elements so called interferents.

- Response time: is the needed time by a sensor to perceive the variation of a physical quantity and to react to it. This requirement may be crucial for those applications where a prompt response is needed.

- Limit of detection (LOD): is defined as the minimum amount of analyte concentration that can be detected by the sensor. For optical sensors, this parameter is calculated considering the ratio between the noise of the measurement and the sensitivity of the sensor.

During the recent last years, we have witnessed a great interest in the development of high-performance lab-on-a-chip (LOC) biosensing devices that are able to replace conventional methodologies currently used, such as PCR or ELISA, which 
are expensive, bulky, time-consuming and lab-centralized (Chin et al., 2007; Wu et al., 2017). The combination of microfluidics and nanoscale transduction elements based on different mechanisms, such as optical, electrical, or mechanical (Estevez et al., 2012; Johnson and Mutharasan, 2012; Vestergaard et al., 2007), allows to perform the required analyses with high sensitivity, a high degree of miniaturization, a high multiplexing level, shorter time to results and requiring very low volumes of sample.

From these biosensors, optical/photonic sensors have gained much interest, due to their extremely high sensitivity, fast response and reduced size, which permits to integrate several sensing structures on a single chip for an ultra-sensitive multiplexed detection. These characteristics make photonic sensors highly attractive for several application areas, such as chemical, biological, defense, industry and biomedical fields. Furthermore, such devices allow the development of label-free assays where the use of radioactive, colorimetric or fluorescent labels, which introduce complexity and loss of activity to biological molecules are avoided (Fernández Gavela et al., 2016; Hunt and Armani, 2010; Zanchetta et al., 2017). Therefore, reduced size advanced label-free biosensors can provide selective, sensitive, fast and direct detection of a certain target analyte at extremely low concentrations.

The healthcare crews, the EU governments and the scientific communities, have approved the implementation of nanotechnology sensing tools for the current and future market, as they show improved features and functionalities compared to the conventional ones. Within those nanotechnology tools, nanophotonics is emerging as one of the highly effective transducers due to the several advantages that it provides, as for example, high sensitivity, reproducibility, label-free detection, compactness, high integration level and low-cost mass production.

The overall objective of this $\mathrm{PhD}$ thesis has been working in the development of an integrated photonic biosensing system suitable for its use in a LOC device in a near future. More specifically, the work carried out has aimed at making a proof of concept of the capability of detecting the selected CVD biomarkers at the required 
concentration using a biosensor combining nanofabricated photonic sensors and a light-assisted biofunctionalization protocol for the immobilization of specific bioreceptors to those biomarkers.

\subsection{CVD biomarkers}

The National Institute of Health Consortium in 2001 defined a biomarker as a "characteristic that is objectively measured and evaluated as an indicator of normal biological processes, pathogenic processes, or pharmacologic responses to a therapeutic intervention" (Arthur J. Atkinson, 2001). Subsequently, in 2009 the American Heart Association outlined the extensive criteria for how newer biomarkers should be evaluated in a standardized fashion before their clinical use can be recommended (Hlatky et al., 2009). More generally, the characteristics of an ideal biomarker to be used for a given purpose in any disease condition, with a special emphasis on CVD, are detailed in some reviews (Hlatky et al., 2009; Wang, 2011).

Biomarkers play an important role in the evaluation and detection of diseases as well as in the development of targeted drug treatments for those disease conditions. In the late phases of drug development, biomarkers can even be helpful in determining the accurate dose for any given drug. In more recent times, biomarkers are being considered as surrogate endpoints for clinical trials as well. More recently, there has been a shift towards the development of precision medicine, especially with a focus on development of drugs and highly sensitive sensing devices.

Furthermore, in hospitals, many patients who present a suspected heart disease have a final diagnosis which excludes an acute coronary event and do not have underlying cardiovascular disease. Here, $5-7 \%$ of patients are turned back home from hospital inappropriately due to this mistaken diagnosis. Hence, an adequate POCT device will enable a fast and accurate diagnosis in an earliest stage, allowing the repetition and analysis of several biomarkers in the same time to ratify the results in a very short time, low cost, in-situ and using only few drops of finger's 
blood. Furthermore, POCT used in healthcare systems will represent a decrease of $25 \%$ from the previously mentioned costs of healthcare service, which represent an amount of $3700 €$ per patient, which means $29 €$ billion of save in total European healthcare system.

Within this context, several CVD biomarkers have been widely studied. In this work, proteins such as C-Reactive Protein (CRP), cardiac troponin (cTn) subunits and Myoglobin $(\mathrm{Mb})$ were considered due to their accuracy, precision, high sensitivity and specificity, which make them ideal CVD biomarkers (Arthur J. Atkinson, 2001).

Some of those biomarkers are not highly specific for cardiovascular diseases but very important indicators (as for example, CRP). Among all the previously mentioned biomarkers Myoglobin, cTnI and cTnT were highly considered for our sensing tool. Note that the biomarkers levels increase notably when a heart attack of the myocardium happens, showing different values depending on the time after a cardiovascular episode. The CVD biomarkers levels evolution versus time are depicted in figure 1.2 .

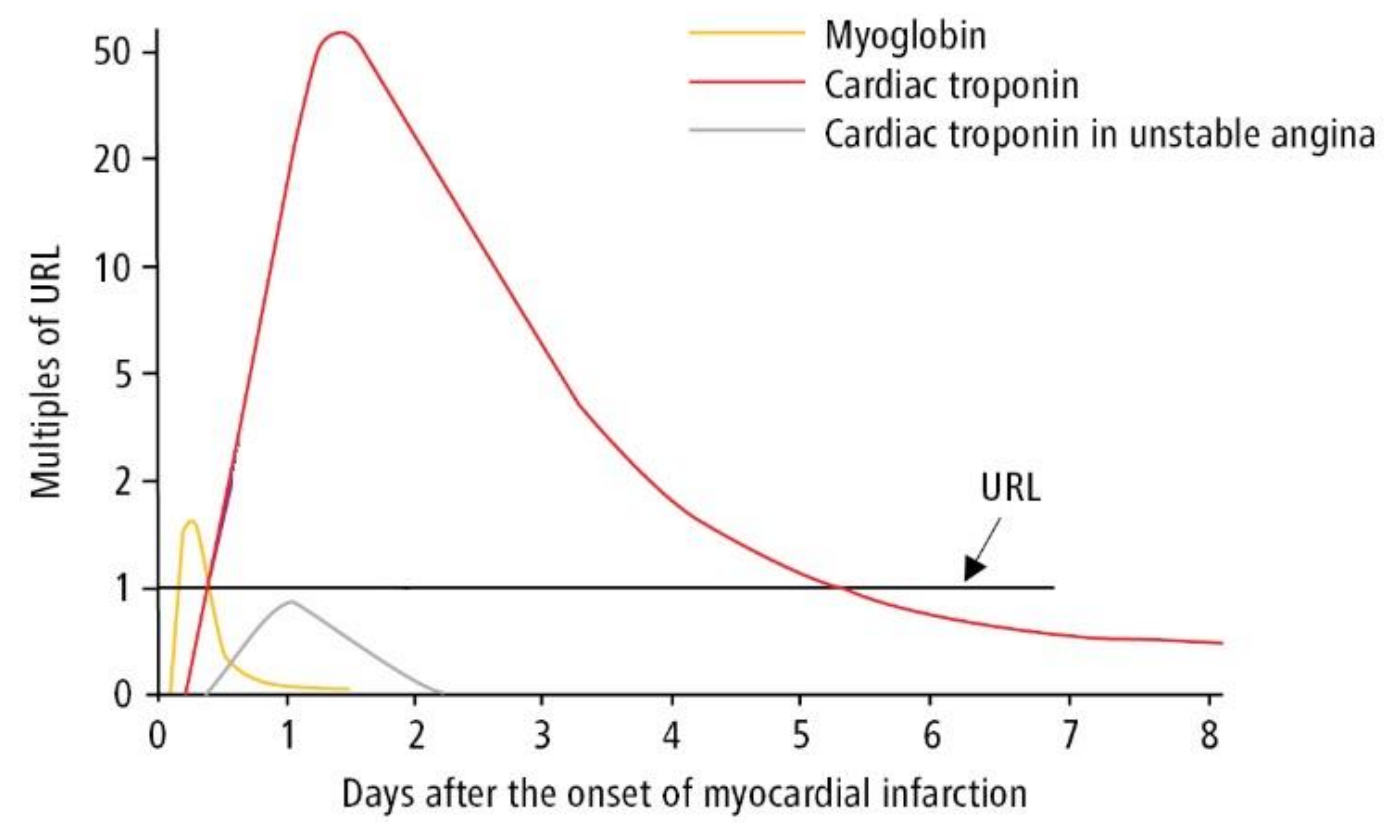

Figure 1.2. Release timing of biochemical markers following acute myocardial infarction in units of release. Adapted from Clin Chem. 1999;45(7):1104-1121. 
Furthermore, the development of a sensing device capable to measure in real-time several biomarkers at the same time is of high interest, since the multiplexed systems are more accurate.

\section{C-Reactive Protein (CRP)}

CRP is a pentagonal protein (see figure 1.3) with about $126 \mathrm{kDa}$ molecular weight, which is considered as a general marker for inflammation and infection. Therefore, it can be used to determine the risk of suffering an acute myocardial infarction. Considered as a member of the acute phase reagents class or acute phase protein, CRP levels increase dramatically during the bodily inflammatory processes. From here, high CRP levels can have multiple causes related with inflammatory processes, so it is not a very specific test for CVD diagnosis. However, the finding of a CRP concentration higher than $2.4 \mu \mathrm{g} / \mathrm{mL}$ doubles the risk of suffering a coronary event respect having concentrations lower than $1 \mu \mathrm{g} / \mathrm{mL}$; so, despite not being very specific, it can guide the diagnosis and help on establishing the risk (StOnge et al., 2009). Normal CRP values, which might vary from one laboratory to another, are typically in the range of $0.25 \mu \mathrm{g} / \mathrm{mL}$ to $3 \mu \mathrm{g} / \mathrm{mL}$.

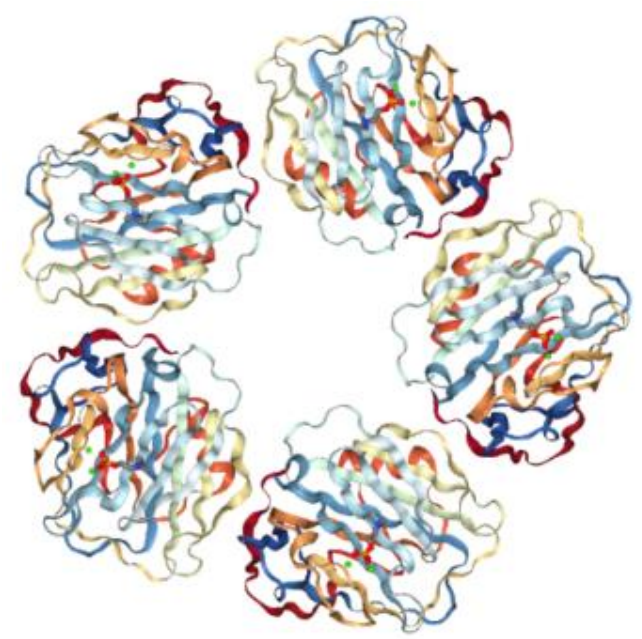

Figure 1.3. Human C-Reactive Protein. Image source RCSB (PDB code 1B09). 
Myoglobin ( $\mathrm{Mb})$

Myoglobin is a muscular hemoprotein, functionally and structurally like hemoglobin, whose function is the storage of oxygen (Collman et al., 1976). With a molecular weight of only $17.2 \mathrm{kDa}$, myoglobin is a small protein constituted by a polypeptide chain of 153 amino acid residues and by a "heme" group that contains an Iron ion (Fe (II)) (see figure 1.4). The heme group is found within a hydrophobic cavity of the protein. This non-polypeptide unit is bound (non-covalently) to myoglobin and is essential for the biological activity of $\mathrm{O}_{2}$ binding of the protein (Ordway and Garry, 2004; Zaia et al., 1992). Myoglobin is released from damaged muscle tissue, which has very high concentrations of myoglobin. Normal myoglobin range is between 25 to $72 \mathrm{ng} / \mathrm{mL}$ and concentrations above $85 \mathrm{ng} / \mathrm{mL}$ are considered abnormal (again, these are guide values, since they can slightly change from one lab to another). As CRP, Myoglobin is a potential marker for cardiac injuries, but taking into account that its levels can be varied by other factors such as inflammatory and degenerative muscle diseases (Lewandrowski et al., 2002).

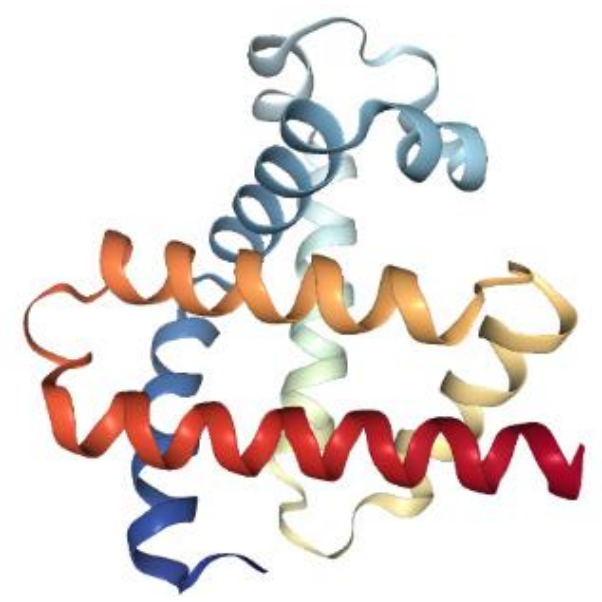

Figure 1.4. Human myoglobin protein. Image source RCSB (PDB code $3 R G K)$.

\section{Cardiac troponin (cTn)}

With a molecular mass of approximately $63 \mathrm{kDa}$, cardiac troponin is a complex composed of three protein subunits (see figure 1.5), having a different role in the 
myocardial contraction (Gomes et al., 2002). Cardiac troponin T (cTnT) is a tropomyosin-binding subunit that regulates the interaction of the troponin complex with the thin filaments of the cardiac muscle. Cardiac troponin I (cTnI) inhibits the interaction between myosin and actin, responsible of muscle contraction. And finally, cardiac troponin $\mathrm{C}(\mathrm{cTnC})$ is a $\mathrm{Ca}^{2+}$ binding subunit responsible of regulating the muscle contraction depending on the $\mathrm{Ca}^{2+}$ concentration. The binding of $\mathrm{Ca}^{2+}$ to cTnC produces a conformational change that reduces the inhibition of cTnI and thus leads to muscle contraction. That conformational change produced by cTnC is reversed when $\mathrm{Ca}^{2+}$ levels are restored, thus creating the contractionrelaxation cycle (Alves et al., 2014; Willott et al., 2010).

When damage of the myofilaments of the cardiac muscle is produced, a break of the troponin complex takes place, leading to the release of the troponin subunits (cTnT, cTnI and cTnC) to the blood stream. Thereby, the levels of these cardiac troponin subunits in blood increases and so can be used as a marker of myocardial damage, especially cTnI (Bagai et al., 2015). Concentrations of cTnI subunit over $10 \mathrm{ng} / \mathrm{mL}$ specifically indicates CVD clinical sign. Note that with $\mathrm{Mb}$ and CRP, cTnI and cTnT are the most specific biomarkers for CVD diseases, from here the high interest to develop a LOC device of high sensitivity for this biomarker.

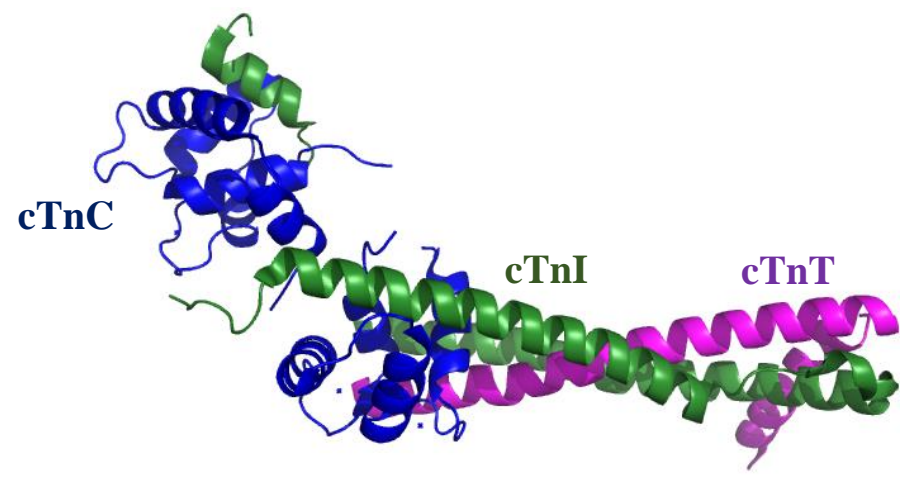

Figure 1.5. Human core cardiac troponin complex in the calcium saturated form. Blue $=$ Troponin $\mathrm{C}$, Green $=$ Troponin I, Pink $=$ Troponin T. Image source RCSB (PDB code 4Y9.9

Within the field of CVD biomarkers detection, several sensing methodologies have been studied. For example, we can mention those based on luminescence and 
colorimetric methods for cardiac marker detection. Here, luminescent signal is generated by the action of an enzymatic labelled antibody in the presence of a luminogenic substrate, while in the case of electroluminescence, the luminescent signal is induced due to an electron transfer reaction of the luminescent bioreceptor immobilized near the proximity of an electrode surface (Cai et al., 2018). This methodology was successfully demonstrated for CRP detection. From here, electroluminescence methods are currently less extensively considered by researchers for CVD markers detection at the expense of nanocompounds sensing methods (Bakirhan et al., 2018). Based on the ECL of CdS nanocrystals, a novel label-free ECL biosensor for the detection of low-density lipoprotein (LDL) as CVD biomarker has been developed by using self-assembly and gold nanoparticle amplification techniques. Unfortunately, no multiplexed detection versatility in this case was offered. Finally, the electrochemical sensing methods. Typically, electrochemical biosensors use an electrode transducer to detect the signal generated following specific binding or catalytic reactions of surface modifier biomaterials such as aptamers, enzymes, antibodies or nucleic acids on the surface of a metal or carbon electrode (Zhu et al., 2015). Note that electrochemical techniques attracted considerable attention because of special features such as simplicity, sensitivity and enable the performance of rapid analysis, but they have the drawback of being lab centralized and sometimes expensive. Whereby, the need of a new sensing method capable to detect several biomarkers in parallel in a short time, cheap and integrable in a POCT device.

\subsection{Evanescent wave photonic biosensors}

Within optical biosensors, those based on evanescent wave photonics are emerging to be the most prominent and interesting ones, due to their high versatility, sensitivity, real-time and label-free detection, thus attracting high interest to become real POCT devices in the near future. 
The detection principle in this type of photonic biosensors consists in the quantification of the variation in the effective refraction index that occurs when an alteration of the chemical or physical parameters is produced on the surface of the structure. This variation is due to the interaction of the optical wave with the medium surrounding the sensor surface. When an optical wave is traveling through the core of an optical waveguide, a fraction of its energy propagates to the surrounding medium, which is known as evanescent wave. This evanescent wave is highly intense in the sensor surface and decreases its intensity exponentially when $z$ increases as depicted in figure 1.6.

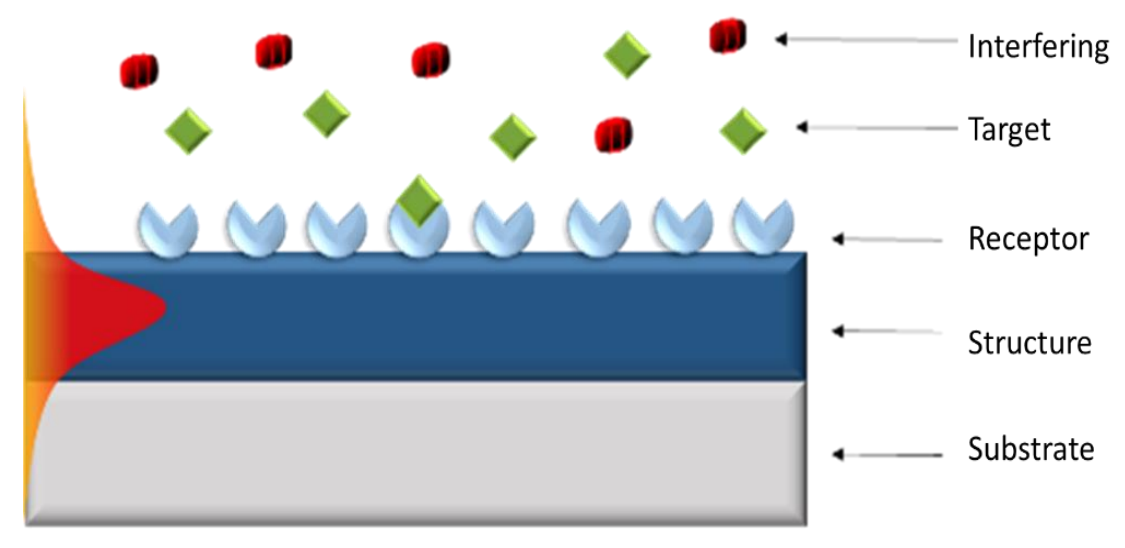

Figure 1.6. Schematic representation of the working principle of an evanescent wave photonic sensor.

The evanescent wave is the main mechanism to detect the effective index changes of the medium in a sensing process. Due to its propagation principle, local changes of the effective index caused by the presence of the biomolecules on the surface of the photonic structure are responsible of changes of the optical guiding properties. These changes are related to the sizes and concentrations of the target biomolecules, thus yielding to a quantitative signal. Note that selectivity is provided by the bioreceptor layer immobilized on the surface of the chip (e.g., chemical receptor, nucleic acids, aptamers, antibodies, etc.).

Photonic biosensors based on evanescent wave detection have demonstrated their outstanding properties, such as an extremely high sensitivity for the direct measurement of biomolecular interactions, in real-time and in label-free schemes. 
In order to convert the effective index variations into a measurable signal such as intensity or frequency variations, different configurations of photonic structures have been developed. Among the most common configurations, we can find ring resonators (Sun and Fan, 2011), Mach Zehnder interferometers (Washburn and Bailey, 2011) and periodic structures (Garcia et al., 2008) as depicted in figure 1.7.
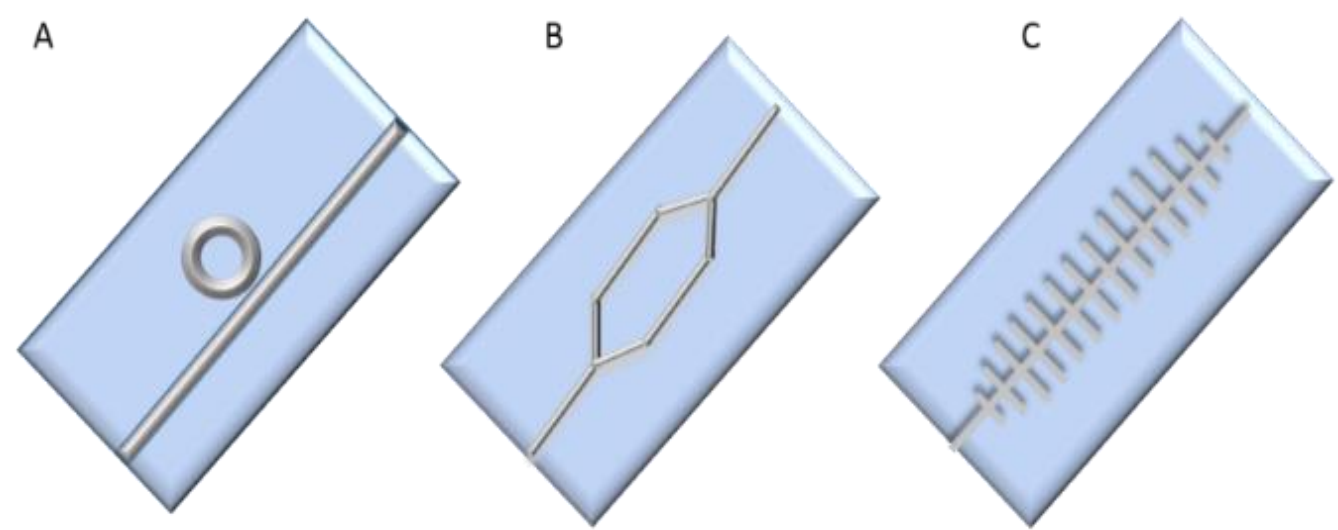

Figure 1.7. A: Ring resonator, B: Mach Zehnder interferometer and C: periodic photonic structure.

From these photonic sensing configurations, those based on periodic configurations are showing an increasing interest because they offer several advantages such as robustness, high sensitivity and easy mass nanofabrication that leads to low-cost photonic sensing chips. Periodic photonic structures, which are sometimes referred to as photonic crystals or as photonic bandgap (PBG) structures, can exhibit what is known as photonic bandgap (PBG): a range of wavelengths in which light propagation is not allowed due to that periodicity of the structure. For sensing purposes, the position of the PBG is shifted when a target analyte/substance interacts with the structure due to the local variation of the refractive index, as it is schematically depicted in figure 1.8. Hence, by monitoring the evolution of the PBG position, we obtain the sensing information. Additionally, note that these periodic structures exhibit the so-called slow wave effect (Povinelli et al., 2005), which leads to a higher interaction between the optical wave and the target analytes/substances, thus increasing the sensitivity. 


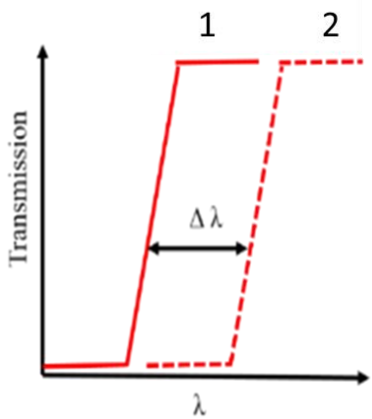

Figure 1.8. Schematic representation of the operation principle of an evanescent wave biosensor based on PBG sensing structures. In the initial state (1), the PBG is located at a certain position; then, the PBG will shift when the target analytes/substances are detected (2).

In this $\mathrm{PhD}$ Thesis, we have made use of $1 \mathrm{D}$ periodic photonic structures, as those previously depicted in figure 1.8. This type of configuration has been selected because it allows creating the PBG structure by simply periodically introducing straight transversal elements in a single mode optical waveguide, thus reducing the complexity in comparison with other $2 \mathrm{D}$ or $3 \mathrm{D}$ periodic configurations.

\subsection{Surface functionalization}

To enhance the interaction between the evanescent wave and the target analytes/substances, the functionalization of the photonic sensor surface is a crucial step. For example, the thinner the bioreceptor layer the higher the wave-target interaction, thus leading to an enhancement of the sensitivity, as schematically depicted in figure 1.9. As previously mentioned, the evanescent field is characterized by an exponential decay from the surface. This causes a strong dependence of the sensitivity with the distance of the target analyte from the structure surface. Since the biorecognition layer represents a new layer formed on the sensor surface, which separates the target analyte from such surface, the interaction between the evanescent wave and the target analyte is weaker as the thickness of the biorecognition layer increases. Some kinds of biofunctionalization techniques require several biofunctionalization steps, deterring from obtaining a 
thin biolayer and leading to a weaker interaction, thus making the detection signal to be minimal or even not measurable.

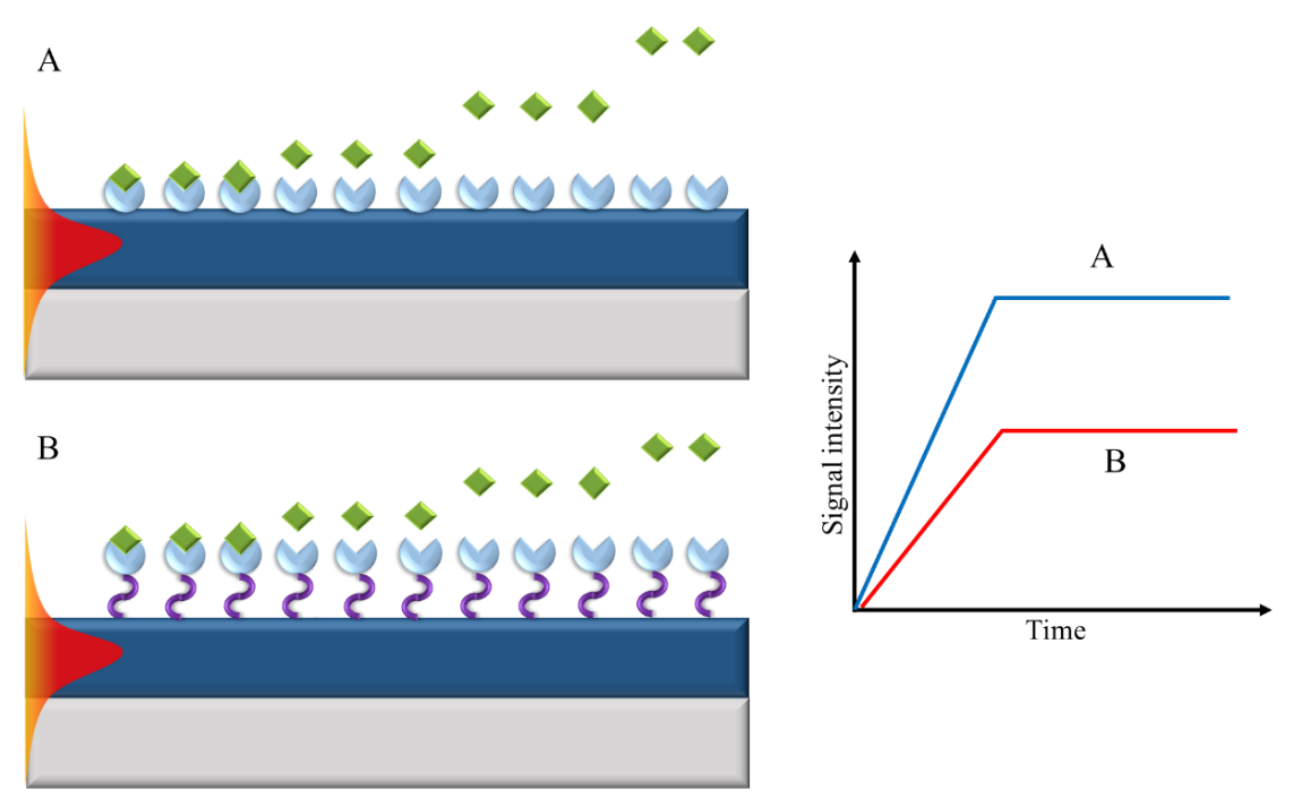

Figure 1.9. (A) Maximal interaction between the evanescent wave and the target analytes due to the creation of a thinner recognition layer, which leads to a higher sensing signal. (B) Lower interaction between the evanescent wave and the target analytes due to the creation of a thicker recognition layer, which leads to a smaller sensing signal.

Among the different procedures for the biofunctionalization of biosensing structures, covalent strategies for the immobilization of bioreceptors provide several advantages in terms of nonspecific interactions restriction, robustness in the surface attachment and thickness reduction of the recognition layer comparing to other strategies based on physical adsorption (Phaner-Goutorbe et al., 2011). Here, for a covalently immobilization performance, it is necessary to design several surface modifications routes taking into account the chemical nature of the surface and depending on the final objective, the kind of the link between the surface and the biomolecule (i.e. through the functional groups of the biomolecule such as Lys, Cys amino acids, carboxyl groups ...) However, covalent surface functionalization strategy is more complex than physical adsorption, involving in our case, the modification of the silicon on insulator (SOI) sensor surface by the incorporation of functional groups able to react with the bioreceptors. Among these functional 
groups, those provided by organosilanes are the most suitable candidates in order to conjugate bioreceptors to glass or similar surfaces (Mujika et al., 2009; Sieval et al., 1998). Several strategies to provide silicon-based surfaces with different terminals groups have been reported in the bibliography, as for example those based on a trifluoroethanol ester and the subsequent thermal acid hydrolysis, those based on the attachment of a photocleavable ester and subsequent photochemical cleavage (Sieval et al., 1998), or those based on the attachment of long chain monolayers (Cricenti et al., n.d.).

Silanization methods are the most common ways to covalently modify siliconbased surfaces in order to selectively attach the bioreceptors and it is much shorter in time than the EDC-NHS protocol for instance. Silane-based surface selfassemblies are extensively reviewed in the literature (Haensch et al., 2010), being trialcoxysilanes such as 3-aminopropyltriethoxy silane (APTES) and 3trimetoxysilyl propyl methacrylate silane (MTPS) those mostly used and reported in the literature for liquid-phase surface functionalization (Halliwell and Cass, 2001; Kim et al., 2009). However, the use of anhydrous solvents in these silanization strategies may lead to some undesirable effects, as for example the polymerization occurring at the free silanol in the solution and/or the silicon surface, altering the thickness of the silane layer, leading to more heterogeneous and irreproducible silane layer (Kim et al., 2009).

In this $\mathrm{PhD}$ work, we have made use of the thiol-ene coupling (TEC) reaction for the biofunctionalization of the photonic sensing chips using half antibodies as bioreceptors. First, SOI surface was silanized to introduce the needed functional groups for a covalent attachment to the bioreceptor. In our case, we use an organosilane in order to introduce the functional groups over the SOI surface (Vinyl, alquinyl, acrylate, thiol, ...). Then, the covalent attachment of the bioreceptor to the chemically modified SOI surface is carried out by means of a click-chemistry reaction which is the thiol-ene reaction. From here, the need of thiolated bioreceptors and vinylated SOI surface or vice versa. 
As it is a click chemistry kind of reaction, TEC is highly effective and does not generate side sub-products. TEC reaction is initiated by UV light which induces the formation of thiol radicals, which reacts with a carbon-carbon double bond $\left(\mathrm{sp}^{2}\right.$ hybridization) leading to a thioether. Moreover, it can be performed in aqueous media, making it ideal for the use of biomolecules. This feature makes the TEC reaction a highly interesting methodology to covalently immobilize the biomolecules for its use on photonic biosensing surfaces.

From the biomolecules employed for the biosensing, the immunoglobulin $\mathrm{G}$ ( $\operatorname{IgG})$ antibodies are the most prominent ones. They are formed by four subunits, two heavy protein chains $(\mathrm{H})$ and two light protein chains (L) separated in two halves. Those two halves are connected in the middle through the hinge region by means of disulfide bonds whose number depends on the species and the subclass of the antibody (Vidarsson et al., 2014). Note that IgGs immobilization is a decisive milestone in the development of a biosensor to detect analytes such as proteins. Another key parameter for the biofunctionalization with IgGs is their oriented immobilization, since antibodies paratopes must be well oriented and available for the target protein to be captured.

Besides the whole antibody, fragments such as Fab and scFab may successfully be employed as probes for proteins biosensing (Crivianu-Gaita et al., 2015; CrivianuGaita and Thompson, 2016). In our context, for the purpose of using the previously mentioned TEC approach conserving an oriented capture site and lower distance from the sensing surface, we consider half antibodies as capture probes to covalently attach to the vinylated surface. To this aim, the disulfide bridge of the hinge region should be selectively reduced in this region resulting into two half antibodies with free thiols (Makaraviciute et al., 2016). Then, the use of the UV light induces the TEC reaction englobing the exposed sulfhydryl ( $\mathrm{SH})$ groups in the half IgG and the available alkene of the triethoxyvinylsilane (TEVS) functionalized PBG sensing surface. This allows an oriented immobilization of the half antibodies with a reduced distance between the capture probe and the photonic sensing surface. 
Figure 1.10 schematically depicts the half IgG to TEVS-activated silicon surface functionalization protocol.

a)

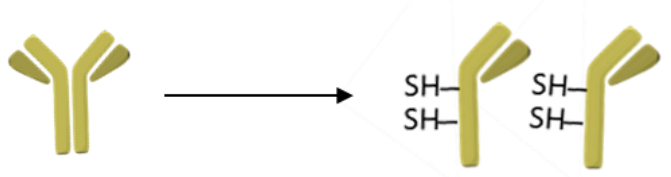

b)

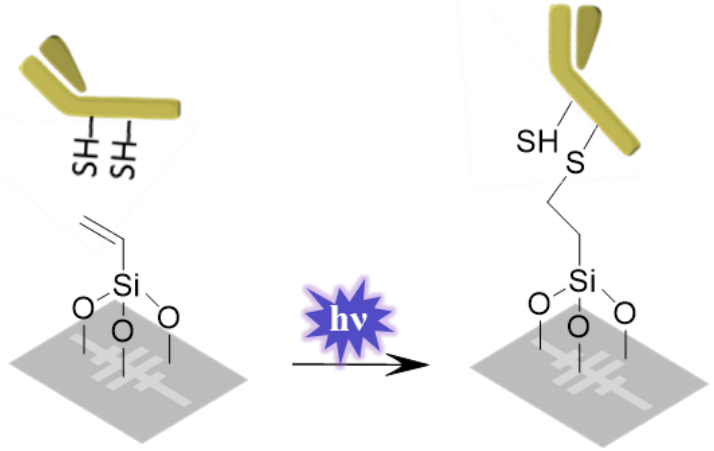

Figure 1.10. (a) First, the IgG is reduced in order to obtain two half IgGs with available SH moieties. (b) The photocatalyzed TEC reaction is employed to induce the reaction between the available SH groups of the half IgGs and the alkene groups on the TEVS-silanized surface in order to covalently attach them to the sensing surface.

\subsection{Framework}

This $\mathrm{PhD}$ Thesis has been carried out at the Nanophotonics Technology Center of the Universitat Politècnica de València (UPV-NTC) in a very close collaboration with the Molecular Recognition group (UPV-SYM) in the frame of the Horizon 2020 European project PHOCNOSIS (Advanced nanophotonic point-of-care analysis device for fast and early diagnosis of cardiovascular diseases). The aim of the PHOCNOSIS project is the development of a highly sensitive, compact and low cost POCT device combining nanophotonic and microfluidic technologies for a non-invasive early diagnosis of CVD. Therefore, the novel technology to be developed in the PHOCNOSIS project would allow a more sensitive, robust and selective analysis for improved clinical decisions through an early and fast diagnosis of CVD at a reduced cost, thus allowing several opportunities to the effective implementation of high-throughput screening programs. This leads to 
better health outcomes, since the proper treatment/response can be applied to the patient in an earlier stage and will contribute to the sustainability of the healthcare system by decreasing the expenditure associated to inappropriate pharmaceutical treatments and with hospitalization. Moreover, this analysis device might also be used for its application in the monitoring and assessment of the therapeutic response of a patient, yielding to the practical implementation of the so-called "personalized medicine".

Within the PHOCNOSIS project, nanophotonic biosensors based on PBG sensing structures are proposed. The high sensitivity provided by nanophotonic sensing technology would allow detecting very low concentrations of the targeted biomarkers, with detection limit (LOD) values in the range of $1 \mu \mathrm{g} / \mathrm{L}(1 \mathrm{ng} / \mathrm{mL})$, making use of sensing structures with an extremely reduced size (only few hundreds of $\mu \mathrm{m}^{2}$, meaning that we can have even thousands of sensing structures on a single chip of few $\mathrm{mm}^{2}$ for a highly multiplexed assay). However, these LOD values will not be enough for the detection of some biomarkers concentrations that need to be detected for the confident diagnosis of CVD at an early stage (in the $\mathrm{ng} / \mathrm{L}(=\mathrm{pg} / \mathrm{mL}$ ) range). So, how will we deal with this issue?

In order to reach those levels, the nanophotonic sensors will be combined with a novel micro-/nanofluidic flow control system in development by one of the PHOCNOSIS consortium members. It is based on a depletion zone isotachophoretic (dz-ITP) filter. This micro-/nanofluidic system will be used to separate, purify and increase the effective concentration of the targeted biomarkers by a factor greater than 1000x. Therefore, the combination of this micro-/nanofluidic flow control system with the nanophotonic sensing structures would allow obtaining a final analysis device providing a DL below $1 \mathrm{ng} / \mathrm{L}(=1 \mathrm{pg} / \mathrm{mL})$, as required clinically.

\subsection{Structure of the Thesis}

After a general introduction of the Thesis frame in chapter 1, chapter 2 presents a theoretical and computational study carried out to identify the binding sites for the 
cardiac Troponin I (cTnI) and for its antibody (anticTnI). Then, molecular docking has been used to test the availability of the system. This study was enriched with a computational study of the selectivity of the anticTnI towards cTnI versus the skeletal Troponin I (sTnI) which is considered the principal interferent for this biomarker. The aim of this study is to demonstrate that a computational binding study is highly interesting to complement and justify the laboratory assays and results.

Chapter 3 presents a study of the functionalization of the photonic sensing structures using half antibodies. Several chemical functionalization trials have been performed and characterized in microarray configuration in order to optimize the biofunctionalization of the photonic chips. Characterization techniques such as Water Angle Contact (WCA), Atomic Force Microscope (AFM), Scanning Electron Microscope (SEM), Infrared Resonance Absorbance Spectroscopy (IRRAS) and Ellipsometry were handled to characterize the functionalized surface and verify that the biofunctionalization process is adequate before transferring it to the photonic chips. Then, PBG sensing structures were nanofabricated in our center clean room facilities and the biofunctionalization process with the half antibodies was carried out in-flow in order to monitor in real-time the UV light assisted immobilization of the bioreceptors when using the TEC reaction.

In chapter 4, and after a Scanning Near-field Optical Microscope (SNOM) characterization study to verify the optimal evanescent wave behavior of the photonic structures, and once the surface chemical functionalization has been optimized, a covalent biofunctionalization method using half antibodies was undertaken. The first system chosen was Bovine Serum Albumin (BSA)/anti Bovine Serum Albumin (antiBSA) since it is a model system already optimized on microarray. Once the biofunctionalization was optimized in microarray, first detection assays by means of PBG structures were performed successfully. Then, a different biofunctionalization protocol based on protein $\mathrm{G}$ physical adsorption was employed over the PBG sensors for the cTnT detection performance in order to 
reach the required CVD biomarkers detection at several concentrations depending on their required concentration ranges.

Finally, chapter 5 is dedicated to general conclusions and future steps to enhance and fulfill the missing aspects for the final device performance.

\subsection{References}

Alves, M.L., Dias, F.A.L., Gaffin, R.D., Simon, J.N., Montminy, E.M., Biesiadecki, B.J., Hinken, A.C., Warren, C.M., Utter, M.S., Davis, R.T., Sadayappan, S., Robbins, J., Wieczorek, D.F., Solaro, R.J., Wolska, B.M., 2014. Desensitization of Myofilaments to $\mathrm{Ca}^{2+}$ as a Therapeutic Target for Hypertrophic Cardiomyopathy With Mutations in Thin Filament Proteins. $\begin{array}{llll}\text { Circ. } & \text { Cardiovasc. } & \text { Genet. } & \text { 132-143. }\end{array}$ https://doi.org/10.1161/CIRCGENETICS.113.000324.

Arthur J. Atkinson, W.G., 2001. Biomarkers and surrogate endpoints: Preferred definitions and conceptual framework. Clin. Pharmacol. Ther. 69, 89-95. https://doi.org/10.1067/mcp.2001.113989. Chin, C.D., Linder, V., Sia, S.K., 2007. Lab-on-a-chip devices for global health: Past studies and future opportunities. Lab Chip 7, 41-57. https://doi.org/10.1039/B611455E.

Avanzas, P., Arroyo-Espliguero, R., Quiles, J., Roy, D., Kaski, J.C., 2005. Elevated serum neopterin predicts future adverse cardiac events in patients with chronic stable angina pectoris. Eur. Heart J. 26, 457-463. https://doi.org/10.1093/eurheartj/ehi111.

Bakirhan, N.K., Ozcelikay, G., Ozkan, S.A., 2018. Recent progress on the sensitive detection of cardiovascular disease markers by electrochemical-based biosensors. J. Pharm. Biomed. Anal. 159, 406-424. https://doi.org/10.1016/j.jpba.2018.07.021.

Bagai, A., Huang, Z., Lokhnygina, Y., Harrington, R.A., Armstrong, P.W., Strony, J., White, H.D., Leonardi, S., Held, C., Van de Werf, F., Wallentin, L., Tricoci, P., Mahaffey, K.W., 2015. Magnitude of Troponin Elevation and Long-Term 
Clinical Outcomes in Acute Coronary Syndrome Patients Treated With and Without Revascularization. Circ. Cardiovasc. Interv. 8. https://doi.org/10.1161/CIRCINTERVENTIONS.115.002314.

Cai, Y., Kang, K., Li, Q., Wang, Y., He, X., 2018. molecules Rapid and Sensitive Detection of Cardiac Troponin I for Point-of-Care Tests Based on Red Fluorescent Microspheres. https://doi.org/10.3390/molecules23051102

Cricenti, A., Longo, G., Luce, M., Generosi, R., Perfetti, P., Vobornik, D., Margaritondo, G., Thielen, P., Sanghera, J.S., Aggarwal, I.D., Miller, J.K., Tolk, N.H., Piston, D.W., Cattaruzza, F., Flamini, A., Prosperi, T., Mezzi, A., n.d. AFM and SNOM characterization of carboxylic acid terminated silicon and silicon nitride surfaces. https://doi.org/10.1016/S0039-6028(03)00999-3.

Crivianu-Gaita, V., Romaschin, A., Thompson, M., 2015. High efficiency reduction capability for the formation of Fab' antibody fragments from $F(a b) 2$ units. Biochem. Biophys. Reports 23-28. https://doi.org/10.1016/j.bbrep.2015.04.004.

Crivianu-Gaita, V., Thompson, M., 2016. Aptamers, antibody scFv, and antibody Fab' fragments: An overview and comparison of three of the most versatile biosensor biorecognition elements. Biosens. Bioelectron. 85, 32-45. https://doi.org/10.1016/j.bios.2016.04.091

European heart network organization, 2017 report.

Estevez, M.C., Alvarez, M., Lechuga, L.M., 2012. Integrated optical devices for lab-on-a-chip biosensing applications. Laser Photon. Rev. 6, 463-487. https://doi.org/10.1002/lpor.201100025.

Fernández Gavela, A., Grajales García, D., Ramirez, J., Lechuga, L., Fernández Gavela, A., Grajales García, D., Ramirez, J.C., Lechuga, L.M., 2016. Last Advances in Silicon-Based Optical Biosensors. Sensors 16, 285. https://doi.org/10.3390/s16030285.

Garcia, J., Sanchis, P., Martinez, A., Marti, J., 2008. 1D periodic structures for slow-wave induced non-linearity enhancement. Opt. Express 16, 3146. 
https://doi.org/10.1364/OE.16.003146.

GBD 2013 Mortality and Causes of Death Collaborators, G. 2013 M. and C. of D., 2015. Global, regional, and national age-sex specific all-cause and causespecific mortality for 240 causes of death, 1990-2013: a systematic analysis for the Global Burden of Disease Study 2013. Lancet (London, England) 385, 117-71. https://doi.org/10.1016/S0140-6736(14)61682-2.

GBD 2015 Mortality and Causes of Death Collaborators, G. 2015 M. and C. of D., 2016. Global, regional, and national life expectancy, all-cause mortality, and cause-specific mortality for 249 causes of death, 1980-2015: a systematic analysis for the Global Burden of Disease Study 2015. Lancet (London, England) 388, 1459-1544. https://doi.org/10.1016/S0140-6736(16)31012-1.

Gomes, A. V., Potter, J.D., Szczesna-Cordary, D., 2002. The Role of Troponins in Muscle Contraction. IUBMB Life (International Union Biochem. Mol. Biol. Life) 54, 323-333. https://doi.org/10.1080/15216540216037.

Halliwell, C.M., Cass, A.E.G., 2001. A Factorial Analysis of Silanization Conditions for the Immobilization of Oligonucleotides on Glass Surfaces. https://doi.org/10.1021/ac0010633.

Haensch, C., Hoeppener, S., Schubert, U.S., 2010. Chemical modification of selfassembled silane based monolayers by surface reactions. https://doi.org/10.1039/b920491a.

Hlatky, M.A., Greenland, P., Arnett, D.K., Ballantyne, C.M., Criqui, M.H., Elkind, M.S.V., Go, A.S., Harrell, F.E., Hong, Y., Howard, B. V., Howard, V.J., Hsue, P.Y., Kramer, C.M., McConnell, J.P., Normand, S.-L.T., O’Donnell, C.J., Smith, S.C., Wilson, P.W.F., American Heart Association Expert Panel on Subclinical Atherosclerotic Diseases and Emerging Risk Factors and the Stroke Council, 2009. Criteria for Evaluation of Novel Markers of Cardiovascular Risk. Circulation 119, 2408-2416. https://doi.org/10.1161/CirculationHA.109.192278.

Hunt, H.K., Armani, A.M., 2010. Label-free biological and chemical sensors. 
Nanoscale 2, 1544. https://doi.org/10.1039/c0nr00201a.

Hurst, J.W. (John W., Logue, R. Bruce, E., Schlant, Robert C., E., Wenger, Nanette Kass, E., 1974. The heart, arteries, and veins. McGraw-Hill.

Johnson, B.N., Mutharasan, R., 2012. Biosensing using dynamic-mode cantilever sensors: A review. Biosens. Bioelectron. 32, 1-18. https://doi.org/10.1016/J.BIOS.2011.10.054.

Kim, M., Long, T.I., Arakawa, K., Wang, R., Yu, M.C., Laird, P.W., 2010. DNA Methylation as a Biomarker for Cardiovascular Disease Risk. PLoS One 5, e9692. https://doi.org/10.1371/journal.pone.0009692.

Kim, J., Seidler, P., Wan, L.S., Fill, C., 2009. Formation, structure, and reactivity of amino-terminated organic films on silicon substrates. J. Colloid Interface Sci. 329, 114-119. https://doi.org/10.1016/j.jcis.2008.09.031

Lee Goldman, by, Schafer, A.I., Schroeder Editor, S., Simel, D., n.d. Cecil Medicine, $24^{\text {th }}$ edition edited part 1: Social and ethical issues in medicine. Part 2: principles of evaluation and management 6: Approach to the patient: history and physical examination.

Leung, W., Chan, C.P., Leung, M., Lehmann, K., Renneberg, I., Lehmann, M., Hempel, A., Glatz, J.F.C., Renneberg, R., 2005. Novel “Digital-Style” Rapid Test Simultaneously Detecting Heart Attack and Predicting Cardiovascular Disease Risk. Anal. Lett. 38, 423-439. https://doi.org/10.1081/AL-200045139

Lewandrowski, K., Chen, A., Januzzi, J., 2002. Cardiac Markers for Myocardial Infarction. Pathol. Patterns Rev. 118, S93-S99. https://doi.org/10.1309/3EK7YVV9-228C-E1XT.

Liu, S., Han, X., Zheng, Z., Chang, Y., 2011. Encephalopathy as an initial symptom of rhabdomyolysis. Neurol. India 59, 764. https://doi.org/10.4103/00283886.86558.

Makaraviciute, A., Jackson, C.D., Millner, P.A., Ramanaviciene, A., 2016. Considerations in producing preferentially reduced half-antibody fragments. 
J. Immunol. Methods 429, 50-56. https://doi.org/10.1016/j.jim.2016.01.001

Mantovani, A., Garlanda, C., Doni, A., Bottazzi, B., 2008. Pentraxins in Innate Immunity: From C-Reactive Protein to the Long Pentraxin PTX3. J. Clin. Immunol. 28, 1-13. https://doi.org/10.1007/s10875-007-9126-7.

Mujika, M., Arana, S., Castaño, E., Tijero, M., Vilares, R., Ruano-López, J.M., Cruz, A., Sainz, L., Berganza, J., 2009. Magnetoresistive immunosensor for the detection of Escherichia coli O157:H7 including a microfluidic network. Biosens. $\quad$ Bioelectron. 24, 1253-1258. https://doi.org/10.1016/j.bios.2008.07.024

Naka, T., Jones, D., Baldwin, I., Fealy, N., Bates, S., Goehl, H., Morgera, S., Neumayer, H.H., Bellomo, R., 2005. Myoglobin clearance by super high-flux hemofiltration in a case of severe rhabdomyolysis: a case report. Crit. Care 9, R90. https://doi.org/10.1186/cc3034.

Ordway, G.A., Garry, D.J., 2004. Journal of Experimental Biology. J. Exp. Biol. 206, 2011-2020. https://doi.org/10.1242/jeb.00243.

Pagidipati, N.J., Gaziano, T.A., 2013. Estimating deaths from cardiovascular disease: a review of global methodologies of mortality measurement. Circulation 127, 749-56. https://doi.org/10.1161/Circulation AHA.112.128413.

Phaner-Goutorbe, M., Dugas, V., Chevolot, Y., Souteyrand, E., 2011. Silanization of silica and glass slides for DNA microarrays by impregnation and gas phase protocols: A comparative study. Mater. Sci. Eng. C 31, 384-390. https://doi.org/10.1016/j.msec.2010.10.016.

Povinelli, M.L., Johnson, S.G., Joannopoulos, J.D., 2005. Slow-light, band-edge waveguides for tunable time delays. Opt. Express 13, 7145. https://doi.org/10.1364/OPEX.13.007145.

Proper, K.I., Singh, A.S., van Mechelen, W., Chinapaw, M.J.M., 2011. Sedentary Behaviors and Health Outcomes Among Adults: A Systematic Review of Prospective Studies. Am. J. Prev. Med. 40, 174-182. 
https://doi.org/10.1016/J.AMEPRE.2010.10.015.

Puleo, P.R., Meyer, D., Wathen, C., Tawa, C.B., Wheeler, S., Hamburg, R.J., Ali, N., Obermueller, S.D., Triana, F.J., Zimmerman, J.L., Perryman, M.B.,

Salazar, J., Martínez, M.S., Chávez-Castillo, M., Núñez, V., Añez, R., Torres, Y., Toledo, A., Chacín, M., Silva, C., Pacheco, E., Rojas, J., Bermúdez, V., 2014. C-Reactive Protein: An In-Depth Look into Structure, Function, and Regulation. Int. Sch. Res. Not. 2014, 653045. https://doi.org/10.1155/2014/653045.

Sieval, A.B., Demirel, A.L., Nissink, J.W.M., Linford, | M R, Van Der Maas, J.H., De Jeu, W.H., Zuilhof, H., Sudhölter, E.J.R., 1998. Highly Stable Si-C Linked Functionalized Monolayers on the Silicon (100) Surface.

St-Onge, M.-P., Zhang, S., Darnell, B., Allison, D.B., 2009. Baseline serum Creactive protein is associated with lipid responses to low-fat and highpolyunsaturated fat diets. J. Nutr. 139, 680-3. https://doi.org/10.3945/jn.108.098251.

Sun, Y., Fan, X., 2011. Optical ring resonators for biochemical and chemical sensing. Anal. Bioanal. Chem. 399, 205-211. https://doi.org/10.1007/s00216010-4237-z.Vestergaard, M., Kerman, K., Tamiya, E., Vestergaard, M., Kerman, K., Tamiya, E., 2007. An Overview of Label-free Electrochemical Protein Sensors. Sensors 7, 3442-3458. https://doi.org/10.3390/s7123442.

Vidarsson, G., Dekkers, G., Rispens, T., 2014. IgG Subclasses and Allotypes: From Structure to Effector Functions. Front. Immunol. 5, 520. https://doi.org/10.3389/fimmu.2014.00520

Wang, T.J., 2011. Assessing the role of circulating, genetic, and imaging biomarkers in cardiovascular risk prediction. Circulation 123, 551-65. https://doi.org/10.1161/CIRCULATIONAHA.109.912568.

Washburn, A.L., Bailey, R.C., 2011. Photonics-on-a-chip: recent advances in integrated waveguides as enabling detection elements for real-world, lab-ona-chip biosensing applications. Analyst 136, 227-236. 
https://doi.org/10.1039/C0AN00449A.

Willott, R.H., Gomes, A. V., Chang, A.N., Parvatiyar, M.S., Pinto, J.R., Potter, J.D., 2010. Mutations in Troponin that cause HCM, DCM AND RCM: What can we learn about thin filament function? J. Mol. Cell. Cardiol. 48, 882-892. https://doi.org/10.1016/j.yjmcc.2009.10.031.

Wu, J., Dong, M., Santos, S., Rigatto, C., Liu, Y., Lin, F., Wu, J., Dong, M., Santos, S., Rigatto, C., Liu, Y., Lin, F., 2017. Lab-on-a-Chip Platforms for Detection of Cardiovascular Disease and Cancer Biomarkers. Sensors 17, 2934. https://doi.org/10.3390/s17122934.

Yale, S.H., Bray, C., Bell, L.N., Liang, H., Haykal, R., Kaiksow, F., Mazza, J.J., n.d. Erythrocyte Sedimentation Rate and C-reactive Protein Measurements and Their Relevance in Clinical Medicine.

Yang, Y.-N., Lin, H.-I., Wang, J.-H., Shiesh, S.-C., Lee, G.-B., 2009. An integrated microfluidic system for C-reactive protein measurement. Biosens. Bioelectron. 24, 3091-3096. https://doi.org/10.1016/J.BIOS.2009.03.034

Zacho, J., Tybjærg-Hansen, A., Jensen, J.S., Grande, P., Sillesen, H., Nordestgaard, B.G., 2008. Genetically Elevated C-Reactive Protein and Ischemic Vascular Disease. N. Engl. J. Med. 359, 1897-1908. https://doi.org/10.1056/NEJMoa0707402.

Zaia, J., Annan, R.S., Biemann, K., 1992. The correct molecular weight of myoglobin, a common calibrant for mass spectrometry. Rapid Commun. Mass Spectrom. 6, 32-36. https://doi.org/10.1002/rcm.1290060108.

Zanchetta, G., Lanfranco, R., Giavazzi, F., Bellini, T., Buscaglia, M., 2017. Emerging applications of label-free optical biosensors. Nanophotonics 6 . https://doi.org/10.1515/nanoph-2016-0158.

Zhu, C., Yang, G., Li, H., Du, D., Lin, Y., 2015. Electrochemical Sensors and Biosensors Based on Nanomaterials and Nanostructures. Anal. Chem 87, 249. https://doi.org/10.1021/ac5039863. 


\section{Chapter 2}

\section{Computational study of the}

\section{cardiac troponin I}

\section{interaction}

\subsection{Introduction}

In the myocardial infarction case, a breakage of the cardiac troponin complex takes place, leading to the release of the troponin subunits ( $\mathrm{cTnT}, \mathrm{cTnI}$ and $\mathrm{cTnC}$ ) to the blood stream. Thereby, the levels of these cardiac troponin subunits in blood can be used as an indicator of myocardial damage. However, troponin is also found in the skeletal muscle, where it is also in charge of controlling the muscle contractionrelaxation cycle (Gordon et al., 2000). And as for the cTn, skeletal troponin (sTn) subunits (sTnT, sTnI and sTnC) are also released to the blood stream when the myofilaments of the skeletal muscle are damaged. Due to the similarity between cardiac and skeletal troponin, sTn subunits may interfere in the detection of cTn ones, thus limiting the utility of cTn detection (Eriksson et al., 2003).

Previous works indicate that, from the three cTn subunits, the utility of cTnC for cardiac damage diagnosis is limited by the fact that it presents the same structure than $\mathrm{sTnC}$, with the only difference of the number of $\mathrm{Ca}^{2+}$ binding sites (Yang et 
al., 2009). Regarding TnT and TnI subunits, cTnT presents several specific cardiac isoforms whereas cTnI presents only one. From here, their use in cardiac diagnostic and prognostic applications (Collinson et al., 2015).

Several comparative studies between cTnT cardiac isoforms and cTnI specific cardiac isoform have been carried out, concluding that cTnI exhibits a higher specificity and accuracy than cTnT for the diagnosis of acute myocardial infarction at its early stage (Apple et al., 2012). Therefore, cTnI has become the current goldstandard biomarker in clinical diagnosis for the identification of acute heart failures. However, it may present a high cross reactivity with the sTnI regarding the interaction with cTnI antibody.

Within this context, in this chapter we perform a computational interaction study of a cTnI antibody (anticTnI) with cTnI and sTnI. This study allows obtaining information about the biochemical interactions between them and to computationally compare the binding performance and the selectivity of the anticTnI towards cTnI versus sTnI rather than experimentally. This information can be relevant for the development of analysis systems for myocardial failure diagnosis based on cTnI detection. First, FTSite and FTMap software were employed to analyze the structure of the targets (cTnI and sTnI) and the antibody binding fragment $(\mathrm{Fab})$ structure. After the consideration of several amount of conformations for cTnI and sTnI (more than a hundred), their most stable conformations (i.e., whom possess the lowest total energy) were chosen to determine and characterize the binding regions of these molecules. Next, pyDock and FTDock were used to study the molecular docking of the cTnI-anticTnI and sTnI-anticTnI complexes to determine their most stable predicted conformations. Energies and interactions results provided by the simulations tools for these predicted conformations allowed us to determine the affinity for each of these complexes and thus to perform the selectivity study. In this study, the temperature is considered at $0 \mathrm{~K}$ in vacuum and employing the rigid mode of the molecules to ensure the stability of the cTnI. 


\subsection{Binding sites identification}

The identification of the binding sites is a long-lasting problem to solve for a wide range of applications such as for proteins, antibodies or for new drugs design. Therefore, an accurate method for binding sites identification is of a high interest. The binding sites of proteins contain the so-called hot spots, specific regions that provide major contributions to the binding free energy. These hot spots are constituted by one or several amino acids that are more likely to bind small molecule probes and compounds with high affinity, and hence are the prime targets in several fields such as immunology or drugs design (Yuan et al., 2013). Experimental techniques to determine these interactions occurring within the binding regions, e.g., nuclear magnetic resonance or X-ray crystallography, are highly expensive, time consuming and can be limited by the physical constraints of the protein-solvent system (Allen et al., 1996). Within this context, computational methods allows characterizing these binding regions numerically rather than experimentally so as to predict their probability of recognition and binding with other molecules (Katchalski-Katzir et al., 1992).

FTSite web server is emerging as a potential solution for this issue, since it is based on experimental evidence that ligand binding sites also bind other small organic molecules of various properties such as polarity, size and shapes. FTSite algorithm does not depend on any evolutionary or statistical information. Nevertheless, it may achieve very high experimental accuracy, since it is based on established test sets that have been used for many other binding sites prediction methodologies (Ngan et al., 2012). FTSite is based on the observation that a binding site of a molecule (i.e., protein) includes a potential main hot spot and other secondary spots close enough to be reached by a ligand binding at the main hot spot (Hall et al., n.d.; Ngan et al., 2012). The premise behind is that ligand binding sites also binds small molecules of various shapes and polarity as observed by NMR and X-ray crystallography experiments (Brenke et al., 2009), so it performs a computational mapping of individual places each of different small molecular probe on a dense grid around the protein and finds favorable positions using empirical free energy 
functions. The probes are clustered, and the clusters are ranked on the basis of their free energy average.

Another computational tool similar to FTSite that has been used in this work for the identification of binding sites is FTMap. FTMap web server presents high potential for determining and characterizing binding hot spots using only computational approaches that may complement the experimental methods, hence avoiding their economical and time drawbacks. FTMap is a close computational analogue of the NMR or X-ray crystallography-based screening experiments. A direct binding hot spots information and molecules druggability are provided by FTMap, and can be used for extending fragment hits up to larger ligands (Brenke et al., 2009a).

In the FTMap approach, the server method consists on the distribution of up to 16 cluster probes (acetone, acetaldehyde, acetamide, acetonitrile, benzaldehyde, benzene, cyclohexane, dimethyl ether, $\mathrm{N}, \mathrm{N}$ dimethyl formamide, methylamine, phenol and urea). By varying size, shape and polarity on the target macromolecule surface, it finds the most propitious positions for each probe type before clustering the probes and ranking the clusters depending on their average energy (Hall et al., n.d.). Those probes are used for an initial rigid body docking against the entire protein surface. The "FT" of FTMap stands for the use of Fast Fourier Transform (FFT) methods to quickly sample billions of probe positions while calculating accurate energies based on a robust energy expression (attractive and repulsive Van-der-Waals terms, electrostatic interaction energies and desolvation energies) based on translational ( $0.8 \AA$ translation) and rotational (500 rotations) motion for each location fastening the calculations (Brenke et al., 2009b; Hall et al., 2011; Kozakov et al., 2011). Following the docking of each probe, thousands of poses are energy-minimized and clustered based on proximity. The algorithm generates bound positions for each of those probes using rigid body docking and samples billions of the available probe positions. Here, regions that bind a high variety of the clusters probes are called the consensus sites, and from these consensus sites, the site containing the higher number of probes is the main hot spot. FTMap ranks the consensus clusters by the number of the bonded contacts between the amino 
acids of the proteins consensus sites and all cluster probes rather than by their number, since the former approach provides slightly better binding sites prediction (Ngan et al., 2012b).

The required input for FTSite and FTMap calculations is an X-ray or NMR structure of the molecule (DNA, protein, ...). X-ray structures of the molecules used in this study were obtained from the Research Collaboratory for Structural Bioinformatics (RCSB) protein data bank (pdb) (www.rcsb.org).

So here, the use of two similar computational based tools for the binding sites identification allows us to demonstrate that both computational methods are accurate if the results are in match, so the computational approach can be a useful strategy to address more rational biosensor development narrowing the experimental campaign.

\subsection{Binding sites results}

The IDs of the sTnI and the anticTnI structures from pdb are 1VDI and 4P48, respectively. Note that the only available anticTnI on the database is the polyclonal one belonging to chicken and hence the only possible one to perform this study. For the case of the cTnI, its sequence was extracted from the whole cardiac troponin complex provided by RCSB with the ID 4Y99. Before their application, those molecules were purified by removing those atoms not corresponding with the molecules of interest from the original pdb files and that might be present in the downloaded models (water and ions).

First, the binding sites on the molecules surface were determined for the cTnI, sTnI and anticTnI. Even when the structure of a molecule is determined by crystallography in a complex with a ligand, a complete description of its binding sites with that ligand cannot be determined experimentally because complex structures may not fully exploit the overall properties of the binding site. Moreover, knowledge of the possible binding sites in the structure of a molecule also enables us to analyze and classify them through their binding sites profiles. Figure 2.1 
shows the chosen binding sites for cTnI, sTnI and anticTnI determined using FTSite. The 3D visualizations were carried out by pyMol software. FTSite also provides the amino acids sequences forming the binding sites as depicted in Table 2.1. Note that the represented results belong to the most stable conformations.
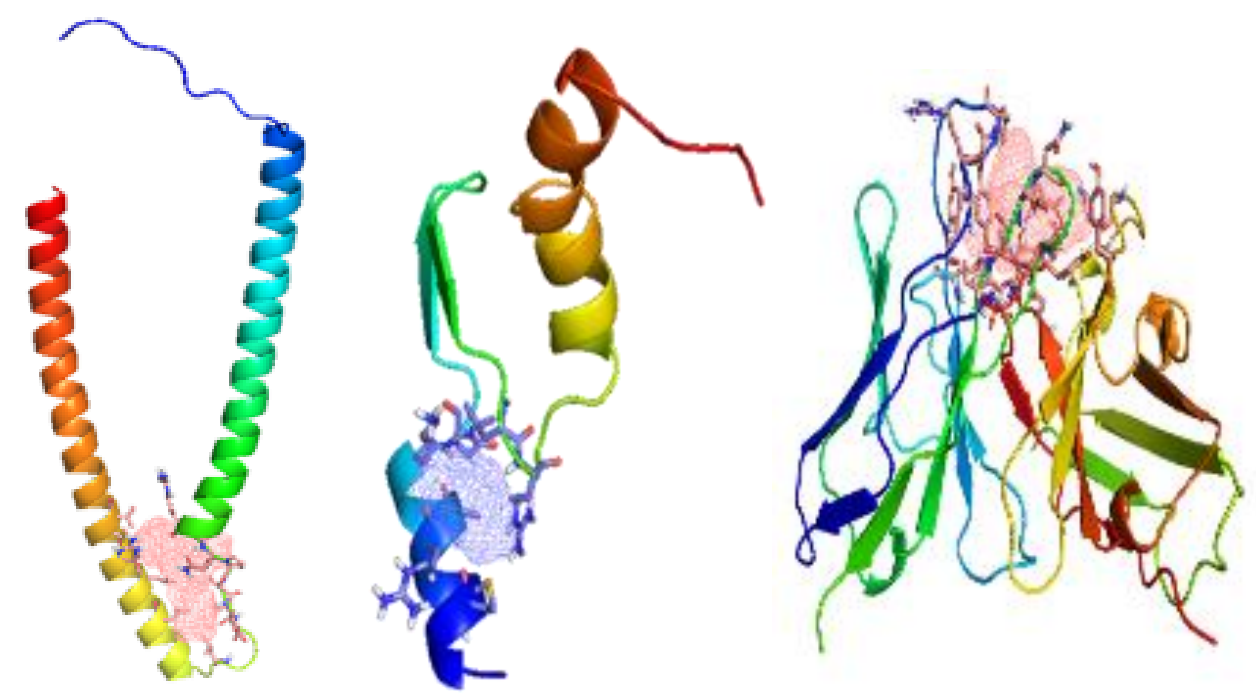

Figure 2.1. FTSite results showing the binding sites (colored grids) of (left) cTnI, (center) sTnI and (right) Fab region of the anticTnI. The visualization has been done using PyMOL.

Table 2.1. Complete description of the amino-acids sequences forming the binding sites given by FTSite for cTnI, sTnI and Fab region of the anticTnI.

\begin{tabular}{crc}
\hline \multicolumn{1}{c}{ cTnI } & sTnI & anticTnI \\
\hline Leu49; Leu52; & Met134; Arg137; Ala138; & Leu2; Arg25; Tyr26; Tyr27; Asp28; \\
Lys72; Arg79; & Asn139; Leu140; Lys141, & Ala44; Pro45; Thr47; Glu51; Lys54; \\
Ala80; Gln81; & Gln142; Val143; Lys145; & Asn58; Asp93; Asp94; Asn95; \\
Pro82; Leu83; & Glu149; Glu151; Lys152; & Asn97; Pro98; Thr99, Phe101; \\
Glu84; Leu93; & Asp153; Leu154; Arg155; & Gly102; Leu130; Leu132; Gly172; \\
Gln99; Leu100; & Asp156; Gly158; Asp159; & Leu173; Glu174; Trp175; Gln180; \\
Arg103; Val104; & Trp160; Arg161; Asn163; & Gly185; Ala190; Ala191; Lys226; \\
Val107; Glu110, & Glu165; Ser168; Gly169; & Asp227; Ser229; Ser230; Asp231; \\
Arg111; Glu115 & Met170; Gly172; Arg173; & Tyr234; Asp237; Ser238; Asp240; \\
& Lys174; Phe177 & Trp242; His244 \\
\hline
\end{tabular}


Once the binding sites sequences were obtained using FTSite, FTMap was executed to determine the number of $\mathrm{H}$-bond interactions between the amino acids comprising the binding site and the organic probe molecules tested by FTMap. Tables 2.2, 2.3 and 2.4 show the H-bond interactions with the probe molecules for cTnI, sTnI and anticTnI, respectively. The first column lists the amino acid number within the binding site, the second lists the amino acid 3-letter code and the third column lists the number of $\mathrm{H}$-bond contacts between the amino acids and the probe molecules.

Table 2.2. cTnI H-bond interaction results between the amino acids from the binding site and the probe molecules as identified by FTMap.

\begin{tabular}{ccc}
\hline cTnI amino acid number & Amino acid 3-letter code & Number of H-bond contacts \\
\hline $\mathbf{4 9 ;} \mathbf{5 2}$; 72; 79; 80; & Leu; Leu; Lys; Arg; Ala; & $66 ; 15 ; 30 ; 556 ; 26 ;$ \\
$\mathbf{8 1 ; ~ 8 2 ; ~ 8 3 ; ~ 8 4 ; ~ 9 3 ; ~}$ & Gln; Pro; Leu; Glu; Leu; & $1510 ; 174 ; 119 ; 918 ; 490 ;$ \\
99; 100; 103; 104; 107; & Gln; Leu; Arg; Val; Val; & $26 ; 761 ; 531 ; 23 ; 830 ;$ \\
$\mathbf{1 1 0} ; \mathbf{1 1 1} ; \mathbf{1 1 5}$ & Glu; Arg; Glu & $431 ; 207 ; 20$ \\
\hline
\end{tabular}

Table 2.3. sTnI H-bond interaction results between the amino acids from the binding site and the probe molecules as identified by FTMap.

\begin{tabular}{|c|c|c|}
\hline sTnI amino acid number & Amino acid 3-letter code & Number of H-bond contacts \\
\hline 134; 137; 138; 139; 140; & Met; Arg; Ala; Asn; Leu; & $102 ; 1 ; 5 ; 6 ; 13$ \\
\hline $141 ; 142 ; 143 ; 145 ; 149$ & Lys; Gln; Val; Lys; Glu; & 133; 84; 63; 1094; 60; \\
\hline $151 ; 152 ; 153 ; 154 ; 155$ & Glu; Lys; Asp; Leu; Arg; & 2014; 11; 3708; 2; 883; \\
\hline $156 ; 158 ; 159 ; 160 ; 161$ & Asp; Gly; Asp; Trp; Arg; & $2 ; 3 ; 3 ; 135 ; 165$ \\
\hline $163 ; 165 ; 168 ; 169 ; 170$ & Asn; Glu; Ser; Gly; Met; & $5 ; 738 ; 1041 ; 981 ; 1020$ \\
\hline $172 ; 173 ; 174 ; 177$ & Gly; Arg; Lys; Phe & $3 ; 618 ; 76 ; 243$ \\
\hline
\end{tabular}


Table 2.4. anticTnI H-bond interaction results between the amino acids from the binding site and the probe molecules as identified by FTMap.

\begin{tabular}{|c|c|c|}
\hline anticTnI amino acid number & Amino acid 3-letter code & $\begin{array}{c}\text { Number of } \mathrm{H} \text {-bond } \\
\text { contacts }\end{array}$ \\
\hline $2 ; 25 ; 26 ; 27 ; 28$ & Leu; Arg; Tyr; Tyr; Asp; & $19 ; 151 ; 233 ; 517 ; 1 ;$ \\
\hline $44 ; 45 ; 47 ; 51 ; 54$ & Ala; Pro; Thr; Glu; Lys; & $2 ; 693 ; 2676 ; 233 ; 11$; \\
\hline $58 ; 93 ; 94 ; 95 ; 97$ & Asn; Asp; Asp; Asn; Asn; & $369 ; 7 ; 282 ; 48 ; 108$ \\
\hline $98 ; 99 ; 101 ; 102 ;$ & Pro; Thr; Phe; Gly; & $338 ; 401 ; 1804 ; 171$ \\
\hline $130 ; 132 ; 172 ; 173 ; 174 ;$ & Leu; Leu; Gly; Leu; Glu; & $43 ; 215 ; 33 ; 646 ; 39$ \\
\hline $175 ; 180 ; 185 ; 190 ;$ & Trp; Gln; Gly; Ala; & $2220 ; 395 ; 14 ; 923$ \\
\hline $191 ; 226 ; 227 ; 229 ; 230$ & Ala; Lys; Asp; Ser; Ser; & $66 ; 24 ; 6 ; 1165 ; 48$ \\
\hline $231 ; 234 ; 237 ; 238$ & Asp; Tyr; Asp; Ser; & $82 ; 1392 ; 816 ; 727$ \\
\hline $240 ; 242 ; 244$ & Asp; Trp; His & $1052 ; 2228 ; 8$ \\
\hline
\end{tabular}

By now, from the more stable conformations of the studied molecules, FTSite provides the amino-acid sequences forming the binding sites and FTMap provides from these binding sites the amino-acids who have a greater number of $\mathrm{H}$-bonds from the interaction with the probes.

Once the binding sites and hotspots are identified, the next step is studying the docking performance between $\mathrm{cTnI}$ and anticTnI from a side and sTnI with anticTnI from the other, in order to determine the most propense docked complexes and to identify the more desirable protein to bind the anticTnI.

\subsection{Molecular docking}

As for binding sites, an accurate scoring of rigid-body docking orientations represents one of the major difficulties in protein-receptor docking predictions. Good discrimination of docking conformations from earliest stages is considered an essential milestone before applying more costly refinement to the correct docking solutions. Here, we have explored simple approaches to score the protein- 
protein dockings implemented in a web server program named pyDock. pyDock is based on Coulombic electrostatics with dielectric constant distance dependence and implicit desolvation energy with atomic solvation parameters for protein-protein docking. pyDock is able to detect a near native solution from thousands (more than 12.000) docking poses and place it within the top 100 lowest energy docking solutions in a large variety of cases (about 56\%), without any additional propitious to confusing information. This makes pyDock a fast tool for better understanding and evaluation of a large range protein-protein docking configurations (Villar et al., 2014). Another parameter to consider is the Van-der-Waals energy. Here, this energy in the scoring of docking configurations is based on the 6-12 Lennard-Jones potential with atomic parameters from AMBER 94 force field (Cornell et al., 1995). As with Coulombic electrostatic potentials, to avoid the noise from the proteinprotein docking surfaces, the interatomic Van-der-Waals energy has been truncated to a maximum of $1 \mathrm{Kcal}^{\mathrm{mol}}{ }^{-1}$. From the other side, FTDock was launched in parallel. FTDock is based on Fast Fourier Transform (FFT), what is used to speed up the calculations by performing them within the Fourier space. Billions of different conformations are examined during this process, from which 10,000 are retained. The scoring criteria for retention of a possible complex is principally based on shape complementarity and also incorporates approximate electrostatics scoring (Li, 2006). Note that for this work, the docking algorithm FTDock was used in order to double check the viability of pyDock, since the same resulting prediction may confirm the correct predicted results.

\subsection{Molecular docking results}

After the identification and mapping of binding sites and the interactions of their constitutive amino acids sequences, the next step is studying the molecular docking for the cTnI and the sTnI to the anticTnI. This process of docking provides the specific interactions details between the proteins and the antibody Fab region taking into account spatial distribution, Van-der-Waals interactions and rotational restrictions, among others. For the docking analysis, FTDock and pyDock were 
launched. FTDock generates several output pdb files with docking probabilities organized from the highest to the lowest probable one. On the other hand, pyDock generates several randomly located pdb files of the docked complexes, and these results are accompanied by other generated files describing energies, positions and angles that is the main key to organize the predicted complexes. The ranking of molecular docking complexes obtained with both methods are the same. Figure 2.2 shows the docked complexes obtained for cTnI-anticTnI and sTnI-anticTnI.
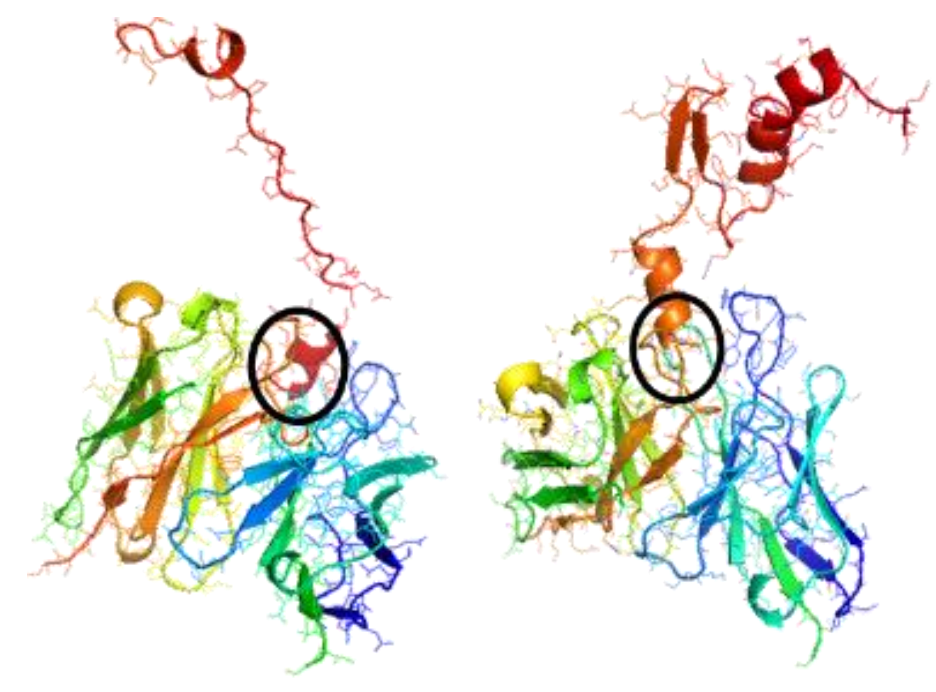

Figure 2.2. Most probable docked configurations of (left) cTnI-anticTnI and (right) sTnI-anticTnI complexes. Binding zones are highlighted with black circles.

Table 2.5 shows the identified H-bond interactions for the cTnI-anticTnI and the sTnI-anticTnI complexes obtained using FTDock and pyDock. Three H-bonds are predicted for both complexes. However, while these H-bonds are involving three different amino acids for the cTnI-antic TnI complex, for the sTnI-anticTnI complex two of them are formed by the same amino acid from the sTnI (Glu149), what is translated into weaker H-bonds than for cTnI-anticTnI formed complex. This fact determines the higher affinity of anticTnI towards cTnI than sTnI. Having identified the most stable predicted structures, the formed cTnI-anticTnI and sTnI-anticTnI complexes were visualized using PyMOL to depict the specific interactions between the amino acids sequences previously identified (see figure 2.3). 
Table 2.5. Identified H-bonds for the cTnI-anticTnI and the sTnI-anticTnI complexes identified using FTDock and pyDock.

\begin{tabular}{cc}
\hline cTnI-anticTnI & sTnI-anticTnI \\
\hline Gln81-Phe101 & Lys145-Lys54
\end{tabular}

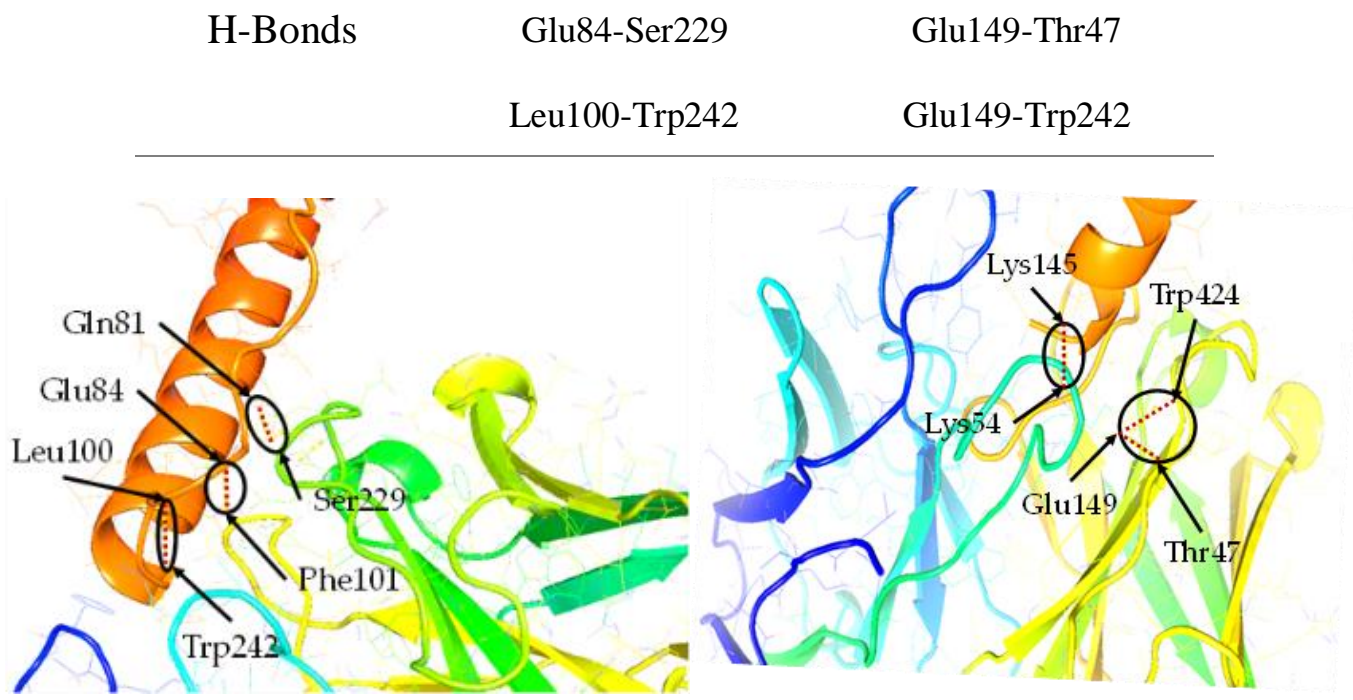

Figure 2.3. Detailed 3D view of the (left) cTnI-anticTnI and (right) sTnIanticTnI docked complexes. H-bonds between the amino acids from the binding sites are depicted with dashed red lines and highlighted with black circles.

Table 2.6 depicts the parameters describing the energies obtained from FTDock for the most stable docking configurations for the cTnI-anticTnI and sTnI-anticTnI complexes. We can see that the total energy of the cTnI-anticTnI complex is considerably lower than for the sTnI-anticTnI complex, what indicates a higher affinity of anticTnI towards cTnI than for sTnI. This confirms the lower affinity for the sTnI-anticTnI complex predicted from the H-bonds data previously shown in Table 2.5 and figure 2.3 .

Table 2.6. Energy data (Kcal/mol) for the most stable docked complexes for cTnI-anticTnI and sTnI-anticTnI.

\begin{tabular}{lcccc}
\hline Complexes & Electrostatic interaction & Desolvation & Van-der-Waals & Total \\
\hline cTnI-anticTnI & -11.866 & -20.190 & 28.475 & -29.208 \\
\hline sTnI-anticTnI & -29.635 & -3.683 & 72.809 & -26.037 \\
\hline
\end{tabular}




\subsection{Conclusions}

In this chapter, we have been able to determine the binding sites of cTnI, sTnI and anticTnI by using FTSite and FTMap. Then, the two troponins were docked with the anticTnI. From the results of energy and the formed hydrogen bonds, we have demonstrated that an affinity study can be performed computationally rather than experimentally, what can dramatically reduce the time and cost when developing immunosensing systems. To our knowledge, this is the first time that this kind of computational affinity study is performed for cardiac troponin I. The obtained computational affinity and selectivity results are highly important, since they compare the suitability of $\mathrm{cTnI}$ and its principal interferent sTnI, to bind anticTnI for a cross-reactivity study, which will allow a better selection of a bioreceptor.

\subsection{References}

Allen, K.N., Bellamacina, C.R., Ding, X., Jeffery, C.J., Mattos, C., Petsko, G.A., Ringe, D., 1996. An Experimental Approach to Mapping the Binding Surfaces of Crystalline Proteins. J. Phys. Chem. 100, 2605-2611. https://doi.org/10.1021/jp952516o

Apple, F.S., Ler, R., Murakami, M.M., 2012. Determination of 19 cardiac troponin I and $\mathrm{T}$ assay 99th percentile values from a common presumably healthy $\begin{array}{llll}\text { population. } & \text { Clin. } & \text { Chem. } & \text { 1574-81. }\end{array}$ https://doi.org/10.1373/clinchem.2012.192716

Brenke, R., Kozakov, D., Chuang, G.-Y., Beglov, D., Hall, D., Landon, M.R., Mattos, C., Vajda, S., 2009a. Fragment-based identification of druggable 'hot spots' of proteins using Fourier domain correlation techniques. Bioinformatics 25, 621-627. https://doi.org/10.1093/bioinformatics/btp036

Collinson, P.O., Gaze, D., Goodacre, S., 2015. The clinical and diagnostic performance characteristics of the high sensitivity Abbott cardiac troponin I 
$\begin{array}{llll}\text { assay. } & \text { Clin. } & \text { Biochem. } & \text { 275-281. }\end{array}$ https://doi.org/10.1016/J.Clinbiochem.2014.12.017

Cornell, W.D., Cieplak, P., Bayly, C.I., Gould, I.R., Merz, K.M., Ferguson, D.M., Spellmeyer, D.C., Fox, T., Caldwell, J.W., Kollman, P.A., 1995. A Second Generation Force Field for the Simulation of Proteins, Nucleic Acids, and Organic Molecules, J. Am. Chem. SOC.

Dennis, S., Kortvelyesi, T., Vajda, S., 2002. Computational mapping identifies the binding sites of organic solvents on proteins. Proc. Natl. Acad. Sci. U. S. A. 99, 4290-5. https://doi.org/10.1073/pnas.062398499

Eriksson, S., Junikka, M., Laitinen, P., Majamaa-Voltti, K., Alfthan, H., Pettersson, K., 2003. Negative interference in cardiac troponin I immunoassays from a frequently occurring serum and plasma component. Clin. Chem. 49, 1095104. https://doi.org/10.1373/49.7.1095

Gordon, A.M., Homsher, E., Regnier, M., 2000. Regulation of Contraction in Striated Muscle. Physiol. Rev. 80, 853-924. https://doi.org/10.1152/physrev.2000.80.2.853

Hall, D.H., Grove, L.E., Yueh, C., Ho Ngan, C., Kozakov, D., Vajda, S., 2011. Robust Identification of Binding Hot Spots Using Continuum Electrostatics: Application to Hen Egg-White Lysozyme. J. Am. Chem. Soc 133, 2066820671. https://doi.org/10.1021/ja207914y

Hall, D.R., Kozakov, D., Vajda, S., n.d. Analysis of protein binding sites by computational solvent mapping. https://doi.org/10.1007/978-1-61779-4650_2

Katchalski-Katzir, E., Shariv, I., Eisenstein, M., Friesem, A.A., Aflalo, C., Vakser, I.A., 1992. Molecular surface recognition: determination of geometric fit between proteins and their ligands by correlation techniques. Proc. Natl. Acad. Sci. U. S. A. 89, 2195-9. https://doi.org/10.1073/PNAS.89.6.2195

Kozakov, D., Grove, L.E., Hall, D.R., Bohnuud, T., Mottarella, S.E., Luo, L., Xia, B., Beglov, D., Vajda, S., 2015. The FTMap family of web servers for 
determining and characterizing ligand-binding hot spots of proteins. Nat. Protoc. 10, 733-755. https://doi.org/10.1038/nprot.2015.043

Kozakov, D., Hall, D.R., Chuang, G.-Y., Cencic, R., Brenke, R., Grove, L.E., Beglov, D., Pelletier, J., Whitty, A., Vajda, S., Designed, S. V, Performed, D.B., 2011. Structural conservation of druggable hot spots in protein-protein interfaces 108. https://doi.org/10.1073/pnas.1101835108/-/DCSupplemental.

Li, H., 2006. A Model of Local-Minima Distribution on Conformational. Bioinformatics 991, 985-991. https://doi.org/10.1002/prot.

Ngan, C.-H., Hall, D.R., Zerbe, B., Grove, L.E., Kozakov, D., Vajda, S., 2012a. FTSite: high accuracy detection of ligand binding sites on unbound protein structures. Bioinforma. Appl. Note 28, 286-287. https://doi.org/10.1093/bioinformatics/btr651

Pagidipati, N.J., Gaziano, T.A., 2013. Estimating deaths from cardiovascular disease: a review of global methodologies of mortality measurement. Circulation 127, $749-56$. https://doi.org/10.1161/CirculationAHA.112.128413

Villar, E.A., Beglov, D., Chennamadhavuni, S., Porco, J.A., Kozakov, D., Vajda, S., Whitty, A., 2014. How proteins bind macrocycles. Nat. Chem. Biol. 10, 723-731. https://doi.org/10.1038/nchembio.1584

Yang, Z., Yamazaki, M., Shen, Q.W., Swartz, D.R., 2009. Differences between Cardiac and Skeletal Troponin Interaction with the Thin Filament Probed by Troponin Exchange in Skeletal Myofibrils. Biophys. J. 97, 183-194. https://doi.org/10.1016/J.BPJ.2009.04.023

Yuan, Y., Pei, J., Lai, L., 2013. Binding site and druggability prediction of protein targets for structure-based drug design. Curr. Pharm. Des. 19, 2326-33. 


\section{Chapter 3}

\section{Real-time monitoring of UV-assisted biofunctionalization by a PBG biosensor}

\subsection{Introduction}

In this chapter, we develop an UV light-induced biofunctionalization protocol for the covalent immobilization of specific bioreceptors over silicon-based biosensing structures. The use of an UV light-induced biofunctionalization method allows a high resolution and spatially selective immobilization. A biofunctionalization protocol based on the thiol-ene coupling (TEC) reaction (Escorihuela et al., 2014), which occurs between thiol and vinyl groups under UV light excitation, has been used to immobilize thiol-terminated bioreceptors over vinyl-terminated SOI PBG sensing structures.

Half-antibodies (hIgG) have been used as bioreceptors for their immobilization over the PBG sensing surface after a selective reduction protocol in the hinge region as schematized in chapter 1 (figure 1.10). This reductive protocol provides free thiol moieties available after the cleavage of their disulfide bridges, allowing us the binding of the half antibodies with oriented paratopes. Note that the use of immobilized hIgG with the biofunctionalization approach described above can also provide several significant benefits such as a higher surface coverage density, a lower thickness of the biorecognition layer and the previously mentioned proper orientation of the antibodies binding sites. The biofunctionalization of the silicon- 
based sensing structures within the photonic chip has been carried out in-flow, while their responses were monitored in real-time, which has allowed us to experimentally confirm the photocatalytic reaction required to immobilize the bioreceptors using the TEC approach.

\subsection{PBG sensors nanofabrication}

SOI is the most employed materials technology in a wide range of nanofabrication approaches and especially in nanophotonics, due to its compatibility with CMOS (Complementary Metal Oxide Semiconductor) fabrication processes from the microelectronics industry. The micro and nanophotonics components developed operate in the infrared (near infrared), usually with a wavelength around $1550 \mathrm{~nm}$. Briefly, the SOI wafer used in this work consists on a $220 \mathrm{~nm}$ thick layer of silicon over a $3 \mu \mathrm{m}$ layer of silicon oxide over around $675 \mu \mathrm{m}$ of silicon that provides more consistency to the whole SOI wafer. Figure 3.1 depicts a simplified scheme of the SOI layers distribution.

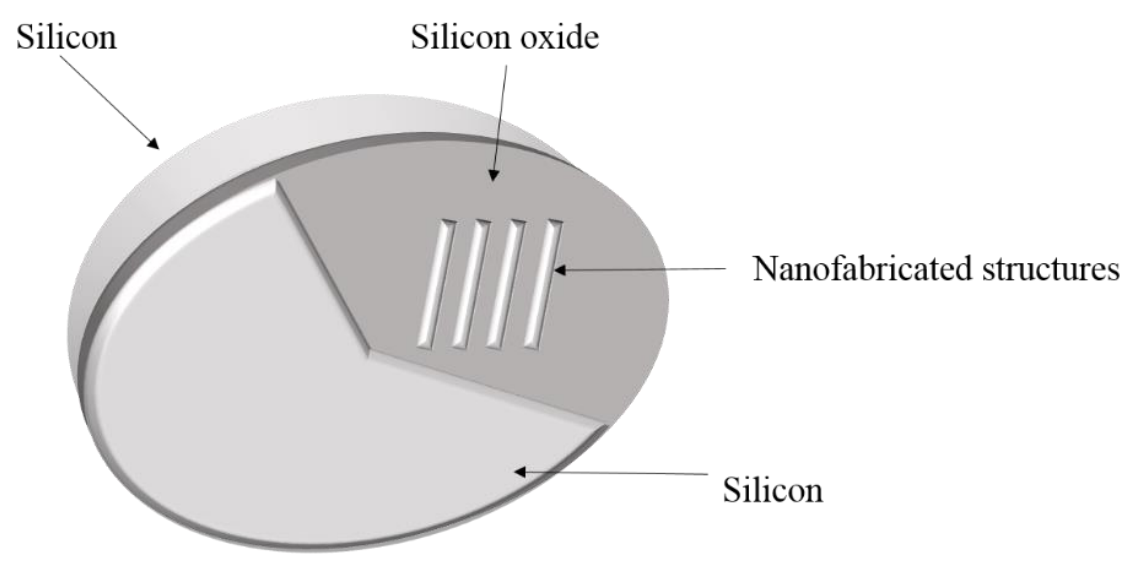

Figure 3.1. Layers distributions of the SOI wafer.

In SOI nanofabricated structures, light is highly confined within the top silicon layer which is highly valuable for photonic structures fabrication. The high confinement of the light is due to the high variation of the refractive index (RI) between the silicon oxide lower cladding $(\mathrm{RI}=1.45)$, the silicon core layer $(\mathrm{RI}=3.46)$ and the air 
upper cladding $(\mathrm{RI}=1)$. Light propagation on a SOI structure is schematized in figure 3.2.

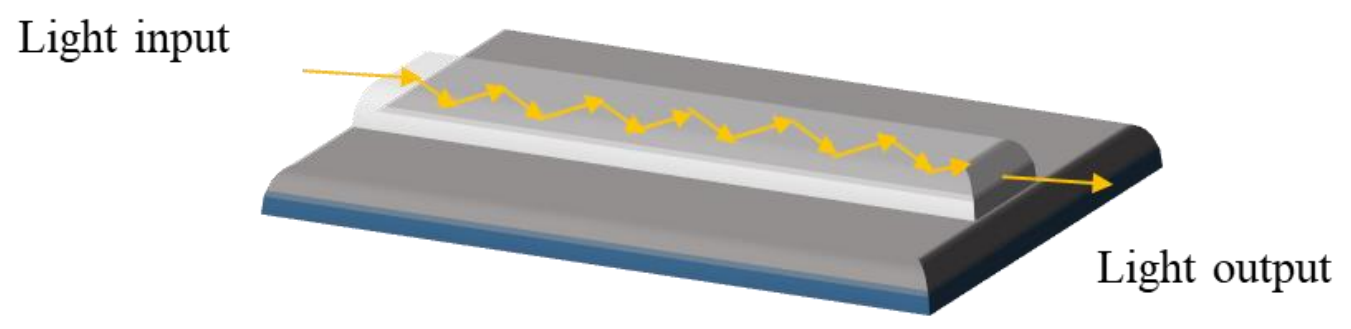

Figure 3.2. Light propagation scheme in a SOI nanofabricated structure.

Another advantage of SOI integrated photonics is the extremely size reduction, which leads to the integration of a large amount of sensing structures in a single SOI piece called photonic chip, thus allowing the possibility to detect simultaneously several biomarkers in a multiplexed chip. Also, mass production nanotechnology is cost effective. Hence, the achievement of an affordable integrated POCT device. The PBG structures used in this work consist on a 1D silicon periodic structure created by the introduction of straight transversal elements in a conventional single mode waveguide, as schematically depicted in figure 3.3, where the main parameters of the structure are also depicted (Garcia et al., 2008; Yablonovitch, 1993).

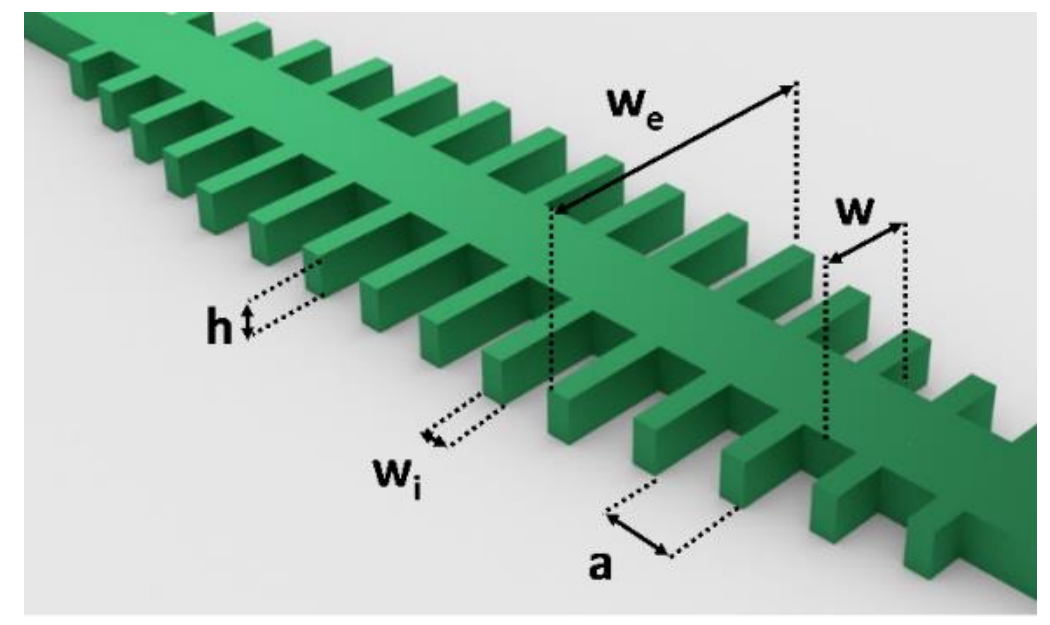

Figure 3.3. Schematic representation of the PBG sensing structure and its structural parameters. 
The design of these PBG structures has been carried out by means of Plane Wave Expansion (PWE) (Johnson and Joannopoulos, 2002) and Finite-Difference TimeDomain (FDTD) (Namiki, 1999) simulations, which were carried out using MIT Photonic Bands (MPB) (Kuchinsky et al., 2002) and CST Microwave Studio, respectively. PWE simulations were used to obtain the band diagrams for the different potential configurations of the PBG periodic structure considering only TE-like modes with an even in-plane symmetry, as PBGs will only appear for that polarization and only that in-plane symmetry will be excited by the access single mode waveguide.

From these simulations, we determined those configurations having their PBG edges located in the 1500-1600 nm wavelength range for air and water upper cladding. For those configurations, FDTD simulations were carried out to obtain their transmission spectral response to select the configuration showing a sharper and deeper PBG edge. The selected parameters for the designed silicon PBG structure are: height $\mathrm{h}=220 \mathrm{~nm}$, waveguide width $\mathrm{w}=450 \mathrm{~nm}$, period $\mathrm{a}=380 \mathrm{~nm}$, transversal elements length $\mathrm{w}_{\mathrm{e}}=1500 \mathrm{~nm}$ and transversal elements width $\mathrm{w}_{\mathrm{i}}$ varying from 80 to $140 \mathrm{~nm}$ to tune the position of the PBGs. Figure 3.4 shows the band diagrams and the simulated transmission spectra of one of those selected configurations when considering air and water upper claddings. As it can be observed, two PBGs appear for this configuration; that appearing between the second and the third bands (PBG2) has been selected as it provides a sharper and deeper PBG. 


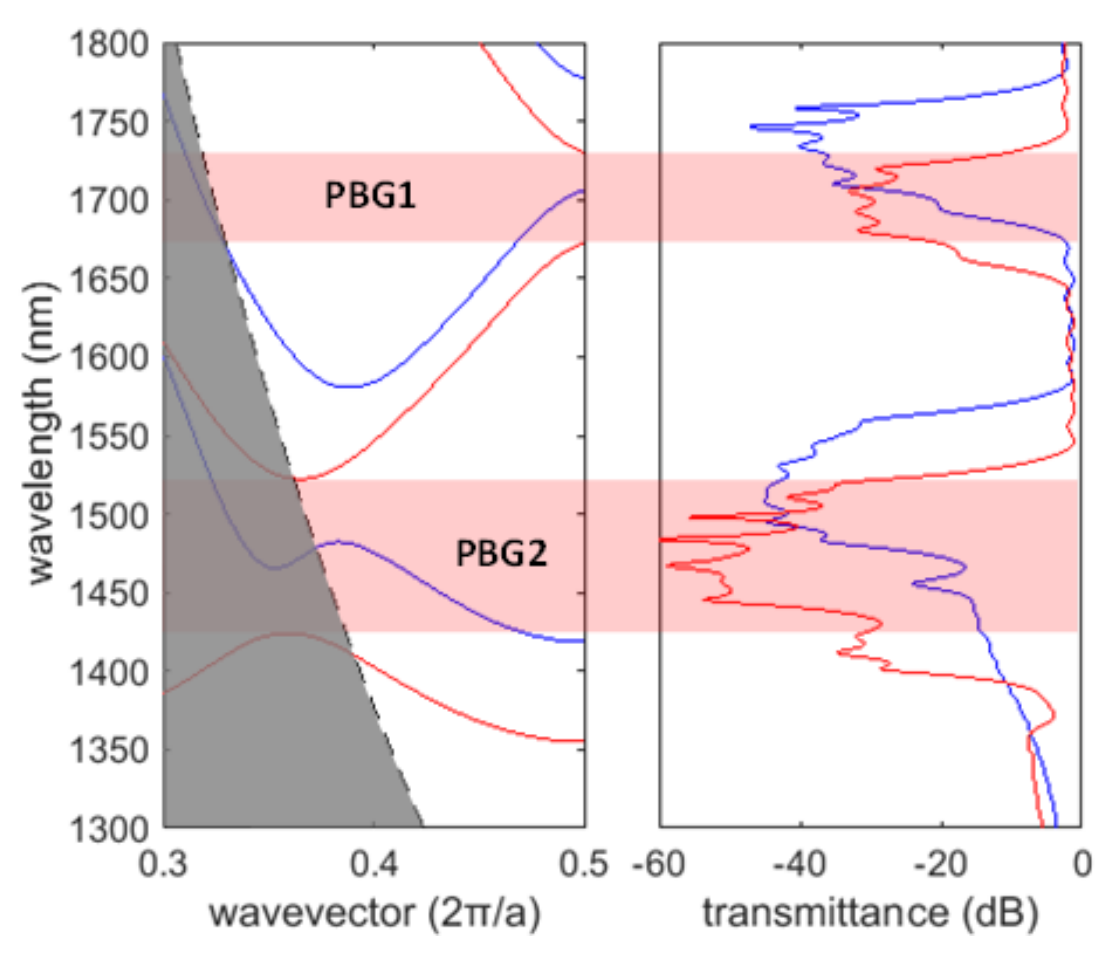

Figure 3.4. (Left) Band diagram and (Right) simulated transmission spectra calculated for the selected configuration of the PBG structure with wi $=120 \mathrm{~nm}$ (red line for an air upper cladding and blue line for a water upper cladding). The red shaded regions depict the two PBGs appearing for this structure for an air upper cladding. The gray shaded region in the band diagram correspond to the modes under the light cone of the $\mathrm{SiO}_{2}$ lower cladding and thus being leaky.

The SOI photonic sensing chip has been created in our clean room facilities using E-Beam Lithography EBL nanofabrication technology (see figure 3.5). After the deposition of a layer of hydrogen silsesquioxane (HSQ) photoresist, the desired structures layout was exposed using EBL. In order to transfer the pattern to the silicon layer, a dry etching using inductively coupled plasma etching was carried out. Here, the remaining photoresist acted as a mask, protecting the covered silicon, while the uncovered part was etched. Finally, the photoresist was removed, and the wafer with the nanofabricated structures was cleaned. 


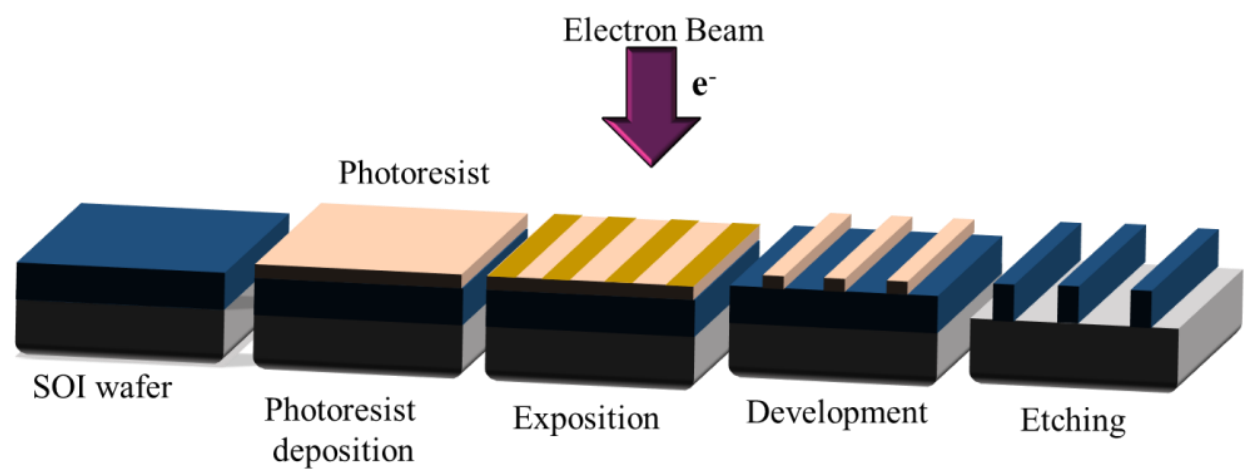

Figure 3.5. Schematic description of the nanofabrication process based on Electron Beam Lithography (EBL).

Figure 3.6 shows several pictures of the fabricated SOI photonic chip. It contains several PBG structures that are used as sensing elements. In these structures, the introduction of a periodic modulation in the refractive index of the photonic structure gives rise to the appearance of a rejected spectral band, the so-called PBG (Povinelli et al., 2005), whose position will depend on the refractive index of the surrounding medium with a high sensitivity. The SOI photonic chip contains 4 groups of 4 PBG sensing structures in each group (16 sensing structures/chip) whose structural parameters are height $h=220 \mathrm{~nm}$, waveguide width $\mathrm{w}=450 \mathrm{~nm}$, period $\mathrm{a}=380 \mathrm{~nm}$, transversal elements length $\mathrm{w}_{\mathrm{e}}=1500 \mathrm{~nm}$, and transversal elements widths ranging from $\mathrm{w}_{\mathrm{i}}=80 \mathrm{~nm}$ to $140 \mathrm{~nm}\left(\mathrm{w}_{\mathrm{i}}=80,100,120\right.$, and 140 $\mathrm{nm}$ for each of the PBG structures within each group). These structural parameters provide PBG edges located in the $1550 \mathrm{~nm}$ wavelength range, where our experimental characterization equipment operates. The separation between the sensor groups is $1.5 \mathrm{~mm}$ in the transversal direction of the photonic chip. The chip is accessed at the input and the output via $70 \mathrm{~nm}$-deep shallow etch $1 \mathrm{D}$ grating couplers. Finally, to provide physical robustness to the fabricated chip with the photonic structures, the chip is covered with a 400 nm-thick $\mathrm{SiO}_{2}$ upper cladding and a $400 \mu \mathrm{m}$-wide channel is opened on it using UV lithography to strip off the oxide from the PBG sensing structures. 


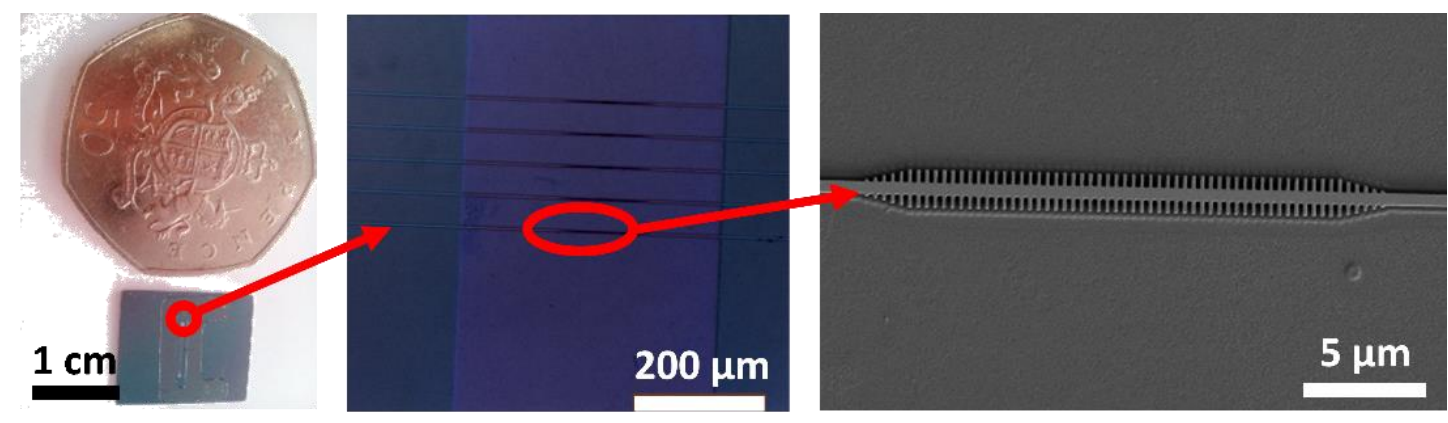

Figure 3.6. (Left) Image of the photonic chip fabricated in SOI technology. The circle depicts the position of one of the PBG sensor groups. (Center) Optical microscope image of a PBG sensor group. The fifth structure on the top up of the group corresponds to a reference waveguide. The channel opened on the $\mathrm{SiO}_{2}$ upper cladding can also be observed. The circle depicts an individual PBG sensing structure. (Right) Scanning Electron Microscope (SEM) image of a fabricated PBG sensing structure.

\subsection{Surface chemistry}

As previously mentioned in chapter 1 , a nanophotonic biosensor is based on the interaction between the evanescent wave and the target analytes. This exponential wave is more intense in the surface of the photonic structure and it weakens as the height increases. From here, the need of a highly thin chemical layer.

Within this context, a trustworthy immobilization of bioreceptors is a crucial step over any surface sensor to achieve the highest sensing performance with the aim to analyze the target samples with any or minimal previous processing (GonzálezGuerrero et al., 2013). To achieve this, we have implemented a functionalization way for the covalent immobilization of the bioreceptors over the photonic sensing area. The proposed method consists in a silanization of the SOI chip surface by means of the Trietoxyvinylsilane (TEVS) which is a trialcoxysilane, usually used in toluene as solvent. Here, we present a novel silanization approach consisting in the use of TEVS in water. The use of water as carrier offers several advantages such as the avoid of the organic waste generation, the decrease of the cost and the most important, leading to the assembly of a compact and homogeneous layer. Furthermore, the cross-linking wards off when the conditions are optimal (TEVS 
$\%$, immersion time, $\mathrm{pH}$, temperature and curing time). Once the silanization step is implemented, the next step is the immobilization of the bioreceptors. Here, we use a covalent linking strategy by employing half antibodies. After their moieties breakage by means of the tris(2-Carboxyethyl) phosphine (TCEP) for the selective reduction of the antibody within the hinge region to obtain free thiols, the TEC reaction is performed to immobilize the half antibodies to the surface via these free thiols. This allows the paratopes of the half antibody to be oriented in the sense of the solution containing the target analytes, which increases the recognition efficiency. UV-light is used to induce the reaction between free thiols from the half antibodies to the alkene groups on the chip surface (photo-catalysis), leading to the TEC chemistry reaction. It is noteworthy to remind that TEC is a type of clickchemistry reaction, which are chemical process reactions that are high yielding, with atom economy, wide in scope and created only by products that can be removed without chromatography. Also, they are stereospecific, simple to perform and can be conducted in easily removable or benign solvents. Yielding to generate substances by joining small units together by means of heteroatom links (C-X-C), with the objective of expanding set of powerful and selective blocks that works in both small and large scale applications (Kolb et al., 2001). Note that cycloadditions, Diels-Alder are the more extensively, whereas $\mathrm{Cu}$ catalyzed azide cycloadditions is one of the most extended example of TEC reaction.

TEC chemistry needs a high thermodynamic driving force, usually greater than 20 $\mathrm{Kcal} / \mathrm{mol}$, difficultly achieved in normal conditions and hence the need of a catalytic reaction. Thus, the use of UV light that induces TEC chemistry reaction between the vinyl group of the TEVS and SH terminated half antibodies seems to be highly effective, selective and does not yields to side products generation, allowing the covalent attachment of the half antibodies to the vinyl groups of the SOI sensing surface. This biofunctionalization approach has been used to immobilize half immunoglobulins $\mathrm{G}(\mathrm{hIgG})$ to the surface of a vinyl-functionalized silicon-on-insulator nanophotonic sensing chip. The response of the sensing structures within the nanophotonic chip was monitored in real-time during the biofunctionalization process, which has allowed us to confirm that the 
bioconjugation of the thiol-terminated bioreceptors onto the vinyl-activated sensing surface is only initiated under UV light photocatalysis.

To immobilize the thiol-terminated bioreceptors onto the silicon-based sensors using the UV light-induced TEC reaction, the silicon-based photonic chip is first silanized using TEVS to obtain a vinyl-terminated compact layer upon its surface (Escorihuela et al., 2012). The silanization process consisted on immersing the SOI photonic chip in 1\% TEVS in Milli-Q water ( $\mathrm{pH}$ adjusted to 8 by adding $100 \mu \mathrm{L}$ of $1 \mathrm{M} \mathrm{KOH}$ in Milli-Q water) for $1 \mathrm{~h}$, then curing it at $110^{\circ} \mathrm{C}$ for $1 \mathrm{~h}$ for condensation and water excess evaporation before the final Milli-Q water rinse of the functionalized chip. Note that before performing the silanization process, the SOI photonic chip was cleaned in a piranha solution $\left(\mathrm{H}_{2} \mathrm{SO}_{4} / \mathrm{H}_{2} \mathrm{O}_{2}: 1 / 3\right)$ for $20 \mathrm{~min}$ and then activated using $\mathrm{O}_{2}$ plasma for $10 \mathrm{~min}$.

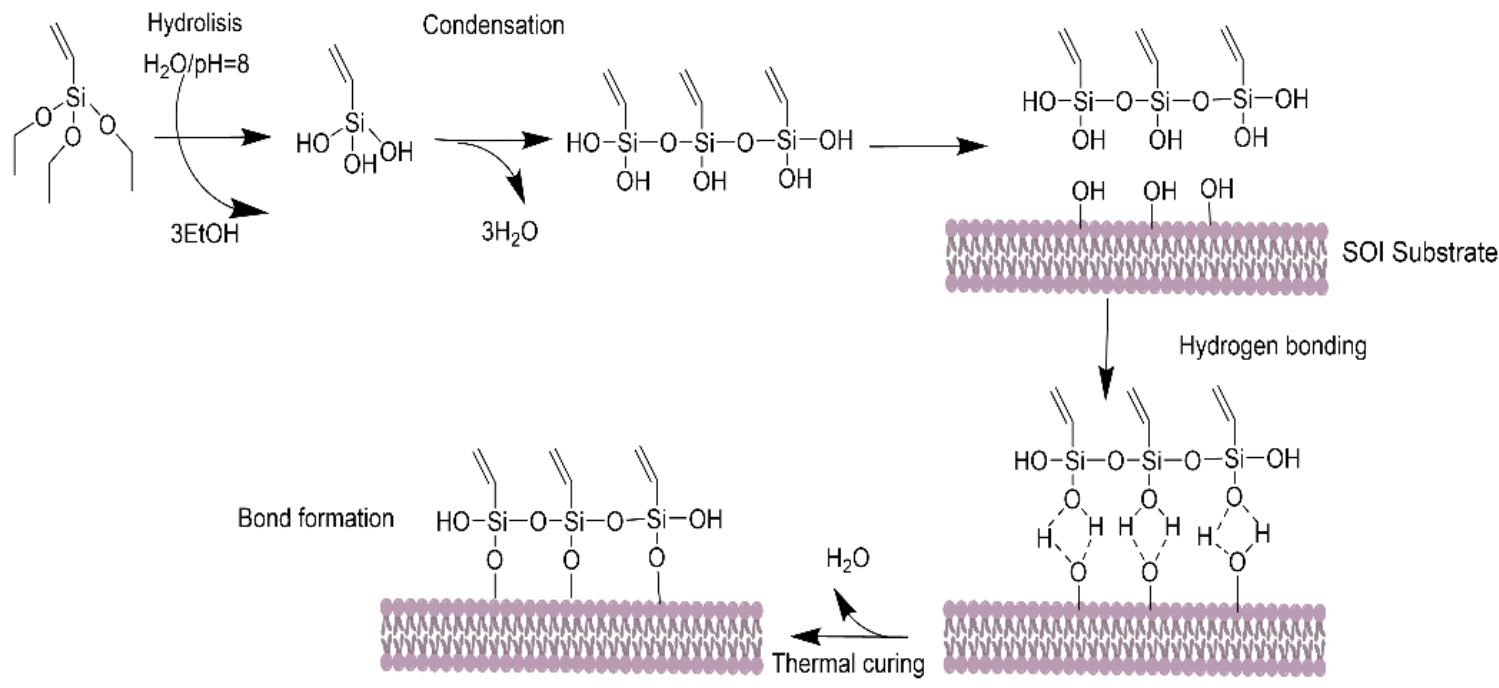

Figure 3.7. Steps of the silanization process used to create a vinyl-terminated monolayer on the surface of the SOI sensing structures. 


\subsection{Surface characterization}

To characterize the initial silanization step, several characterization techniques were employed before and after performing it. First, the water contact angle (WCA) test indicates a significant increase of the hydrophobicity of the surface after performing the TEVS silanization from $\sim 30^{\circ}$ before to $\sim 80^{\circ}$ after silanization as shown in figure 3.8, which confirms the coverage of the SOI surface with the organosilane. Then, IRRAS (Infrared Reflection Absorption Spectroscopy) was employed to characterize the functional groups upon the surface (see figure 3.9). The spectral bands appearing at $1500 \mathrm{~cm}^{-1}$ corresponds $\mathrm{C}=\mathrm{CH}$ whereas the $\mathrm{Si}-\mathrm{CH}$ and the Si-O-Si appears at 1400 and $1200 \mathrm{~cm}^{-1}$ respectively. The spectral bands at $3062 \mathrm{~cm}^{-1}$ and $3020 \mathrm{~cm}^{-1}$ correspond to the $=\mathrm{C}-\mathrm{H}$ asymmetric and symmetric stretching vibrations for the vinyl groups of the TEVS organosilane, which confirms the presence of the vinyl groups upon SOI surface required to perform the proposed light-assisted immobilization of thiol-terminated probes. Finally, the topography of the surface before and after TEVS functionalization was characterized by means of AFM (Atomic Force Microscope) measurements. Figure 3.10 demonstrates that a very low roughness is measured after performing the silanization process, which indicates a high homogeneity and compactness of the deposited TEVS layer. Therefore, all the characterization techniques employed confirm the adequate creation of the TEVS layer on the surface of the SOI chip.

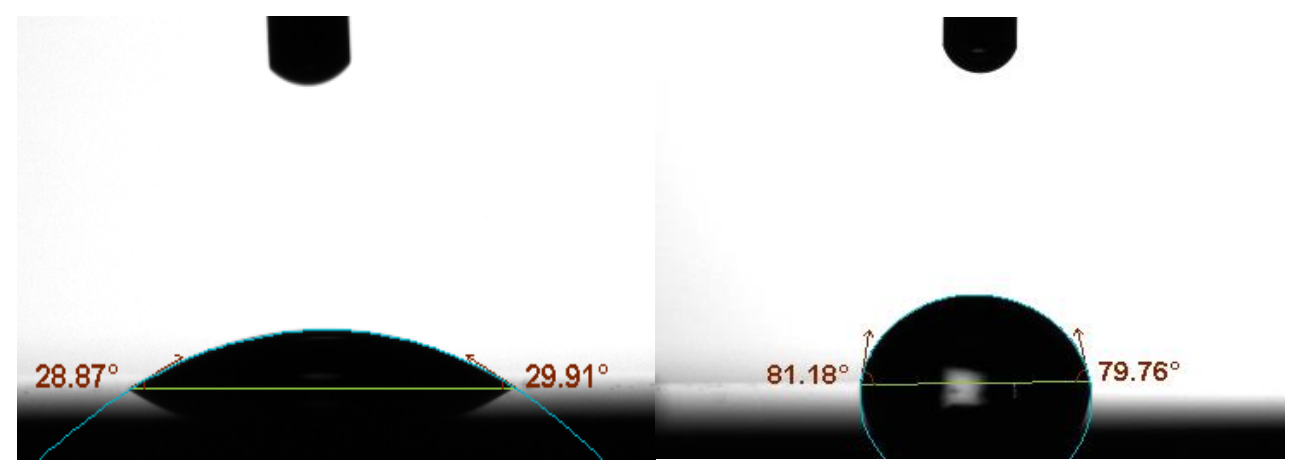

Figure 3.8. (Left) Water contact angle before silanization. (Right) Water contact angle after silanization. 


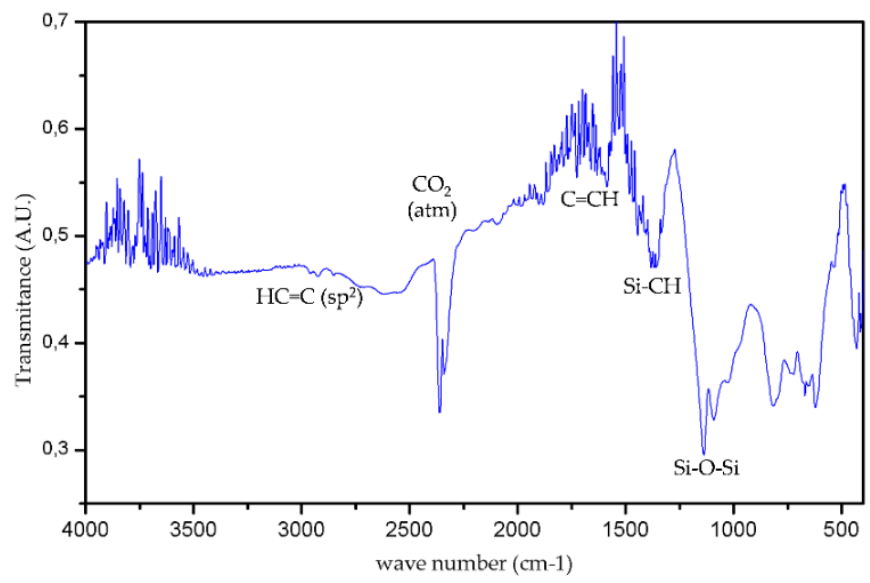

Figure 3.9. Infrared reflection absorption spectroscopy (IRRAS) characterization results of the SOI chip after the TEVS silanization.
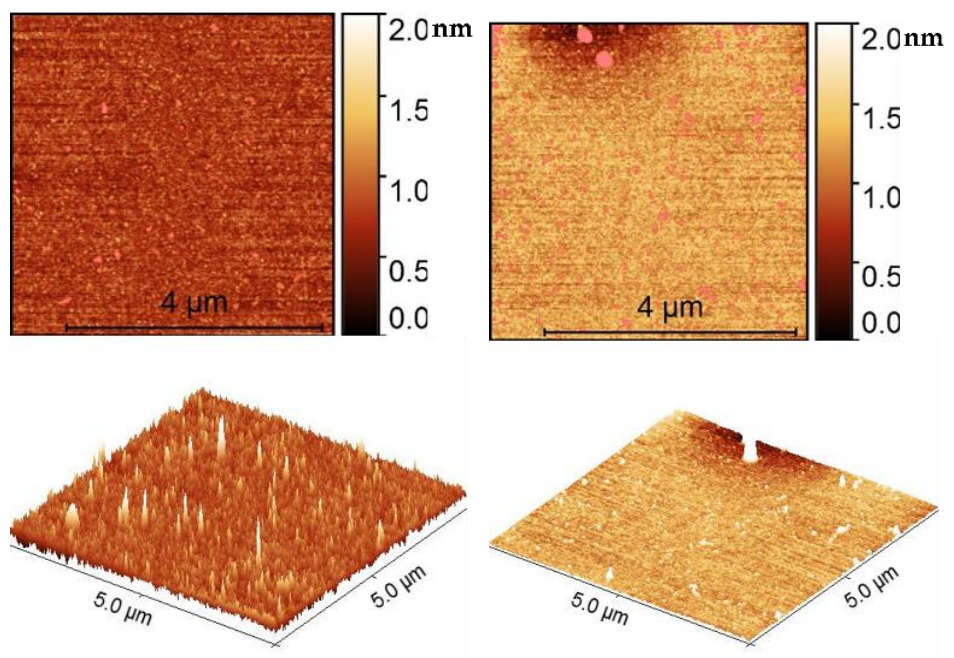

Figure 3.10. Atomic force microscope (AFM) topography characterization of (left) the bare SOI surface and (right) the SOI surface after TEVS silanization. A very low roughness (in the sub-nm range) is measured for both surfaces.

\subsection{UV light-assisted immobilization of half antibodies}

IgGs or antibodies are nowadays the most dominant class of immunoglobulins employed in the biosensing field because of their high immune affinity to proteins. Antibody fragments are emerging also to be successfully employed as biosensing probes, since reductions such as mercaptoethylamine (MEA) and tris(2carboxyethyl) phosphine (TCEP) are protocols that allow obtaining those 
fragments. When employing MEA reduction, an over reduction occurred and disulfide bridges are cleaved driving to a non-selective fragment cleavage, leading to loss of recognition property. In contrast, the TCEP protocol can be optimized in concentrations, time and temperature leading to the identification of the optimal conditions and hence the reach of the maximal number of half IgGs with the highest recognition capability with presence of reduced disulfide bonds within the hIgG moieties (Cline et al., 2004; Makaraviciute et al., 2016).

The thiol-terminated bioreceptors used here are hIgG specific to bovine serum albumin (BSA). These rabbit half anti-BSA antibodies (haBSA) were obtained using TCEP reduction process consisting on the incubation (for $90 \mathrm{~min}$ at $37^{\circ} \mathrm{C}$ ) of the anti-BSA in acetate buffer $(0.15 \mathrm{M}$ sodium acetate, 0.01 M EDTA, 0.1 M sodium chloride, $\mathrm{pH}=4.5$ ) at $4 \mathrm{mg} / \mathrm{mL}$ concentration in the presence of $25 \mathrm{mM}$ TCEP. The corresponding haBSA were purified by employing a $50 \mathrm{kDa}$ centrifugal filter unit and the concentrations of the solutions were determined by employing a NanoDrop spectrophotometer. After this cleavage process, thiol groups from the disulfide bridges turn to be available on the resulting haBSA for their immobilization over the vinyl-terminated surface by means of UV light $(254 \mathrm{~nm})$ photocatalysis, as schematically depicted in figure 3.11. Further details about this TCEP reduction process can be found in (Alonso et al., 2018).

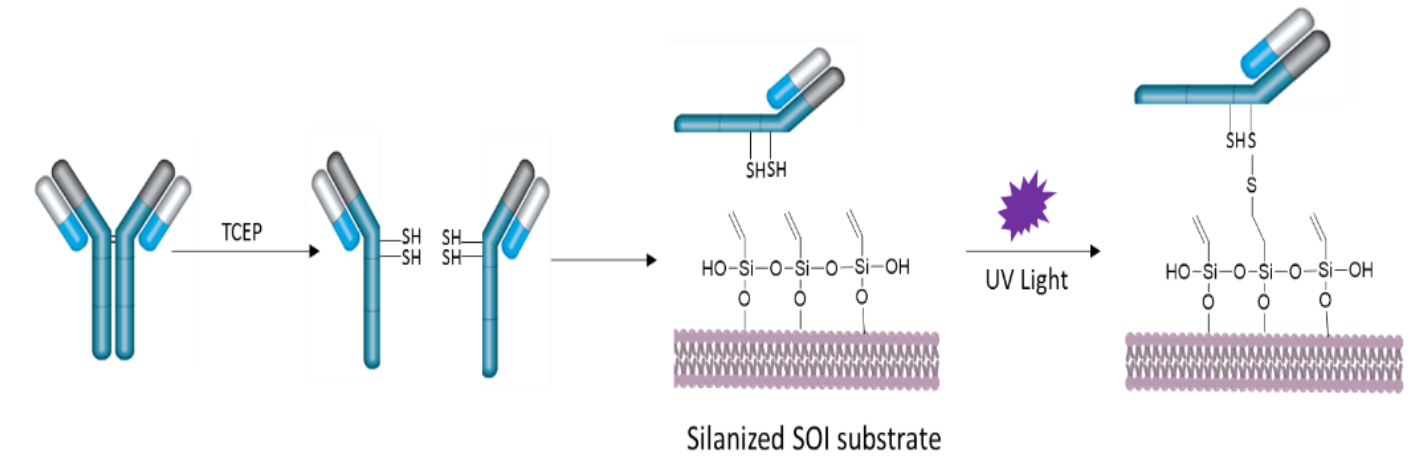

Figure 3.11. Schematic representation of the process used for the UV lightassisted covalent immobilization of the half anti-bovine serum albumin (haBSA) on the SOI sensors surface. 
Once the proper silanization of the chip surface was confirmed, we performed fluorescence microarray tests on planar SOI surfaces to verify the proposed lightassisted TEC immobilization protocol. To this aim, AlexaFluor 647-fluorophore labelled haBSA (haBSA*) at $20 \mu \mathrm{g} / \mathrm{mL}$ nanodrops volume were dropcasted over two different TEVS silanized SOI chips. One of those chips was irradiated with UV light $\left(254 \mathrm{~nm}\right.$ with a power of $\left.6 \mathrm{~mW} / \mathrm{cm}^{2}\right)$ to induce the reaction between the vinyl groups on the surface and the thiol groups from the haBSA*, while the other chip was kept without UV light irradiation. Finally, both chips were thoroughly washed with phosphate buffered saline solution with Tween 20 (PBS-T) followed by a water washing before they were dried. Then the fluorescence was checked by a CCD camera. Figure 3.12 shows the fluorescence results for both chips, where we can clearly see that fluorescence is only observed for the chip being irradiated with UV light, thus confirming the UV-dependent nature of the biofunctionalization process.

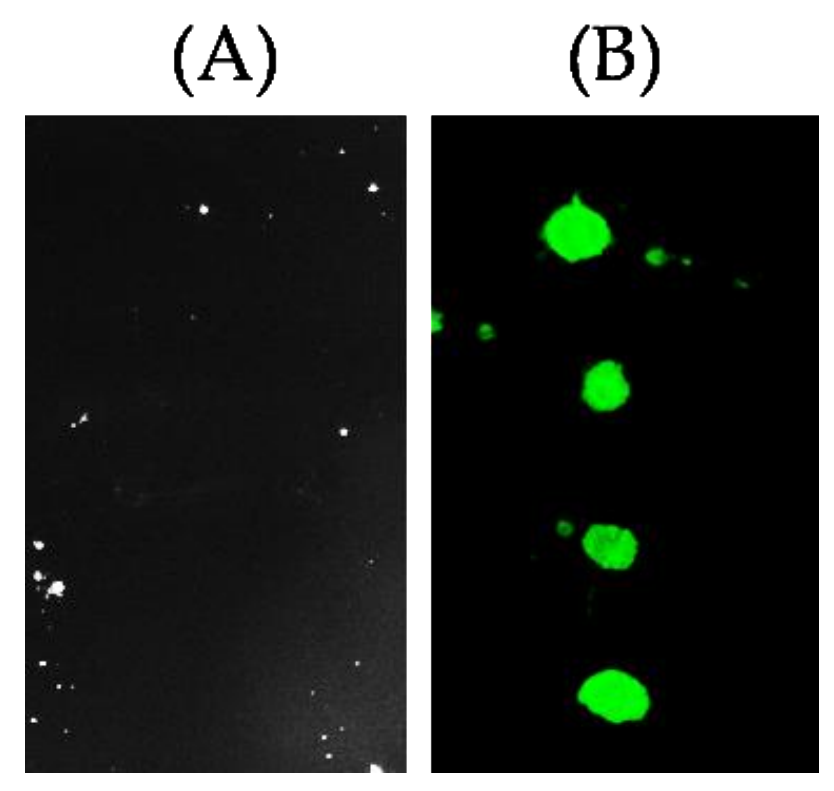

Figure 3.12. Fluorescence microarray measurements employing a CCD camera for: (A) TEVS silanized chip with deposited haBSA* at $20 \mu \mathrm{g} / \mathrm{mL}$ with no UV irradiation and (B) TEVS silanized chip with deposited haBSA* after $254 \mathrm{~nm}$ UV light irradiation (5 s). 


\subsection{Experimental opto-fluidic setup}

In order to deliver the target sample over the chip, a microfluidic flow cell has been used. The microfluidic cell material is a relevant parameter, since the irregularity may lead to bubble air generation or leakage. The material should also be inert and versatile in order to path adaptation of the flow system to the experimental requirements. Within this context, several materials were considered, but polydimethylsiloxane (PDMS) exhibits several advantages. PDMS is an elastomer that has the ability to adopt the shape of the container and surface after curing, what allows to easily fabricate the microfluidic structures by soft lithography with good results, obtaining small drawbacks for the Lab-On-a Chip integration. Moreover, the material is biocompatible due to its inertness, low cost and ease of handle and fabricate. Additionally, PDMS is transparent which makes possible through in irradiation and the observation of the flow evolution through the fluidic system and detect in time any obstruction or leakage. For the microfluidic channel fabrication with PDMS, two principal steps are required: the creation of the mold and the transfer of the design to the PDMS. For the creation of the mold, it has been fabricated in a silicon wafer by patterning the microchannels in "Sukhoy-8" polymer (SU-8), which is a negative photoresist with presence of eight epoxy groups by standard UV lithography. In our case, for the master mold design, we used a $50 \mu \mathrm{m}$ thick SU-8, which leads to $50 \mu \mathrm{m}$ height microchannel after lithography. The transfer of the design to the PDMS was done like following. First, a mixture of $90 \%$ PDMS and $10 \%$ curing agent weight were blended on a Petri. Then, it was degassed using a vacuum chamber to take off all air bubbles that may cause air leakage and/or obstructions into the final microfluidic channel. And finally, the master mold was covered with the PDMS and curing agent mixture and cured in a stove at $110^{\circ} \mathrm{C}$ during $30 \mathrm{~min}$. Then, the master mold is removed and the PDMS to Teflon tubing connections holes are perforated using a puncher and the tubes were connected to a syringe pump. Pumping assays using different solutions were performed to proof the microfluidic efficiency of the channels. 
The ease of PDMS channels fabrication allows the handle of many channels at the same time. Starting by varying parameters until the optimal shapes and dimensions are achieved. More than 50 microfluidic channels were fabricated at the same time, each of them was reused in several experiments. The real microfluidic channel used in the experiments is depicted in figure 3.13.

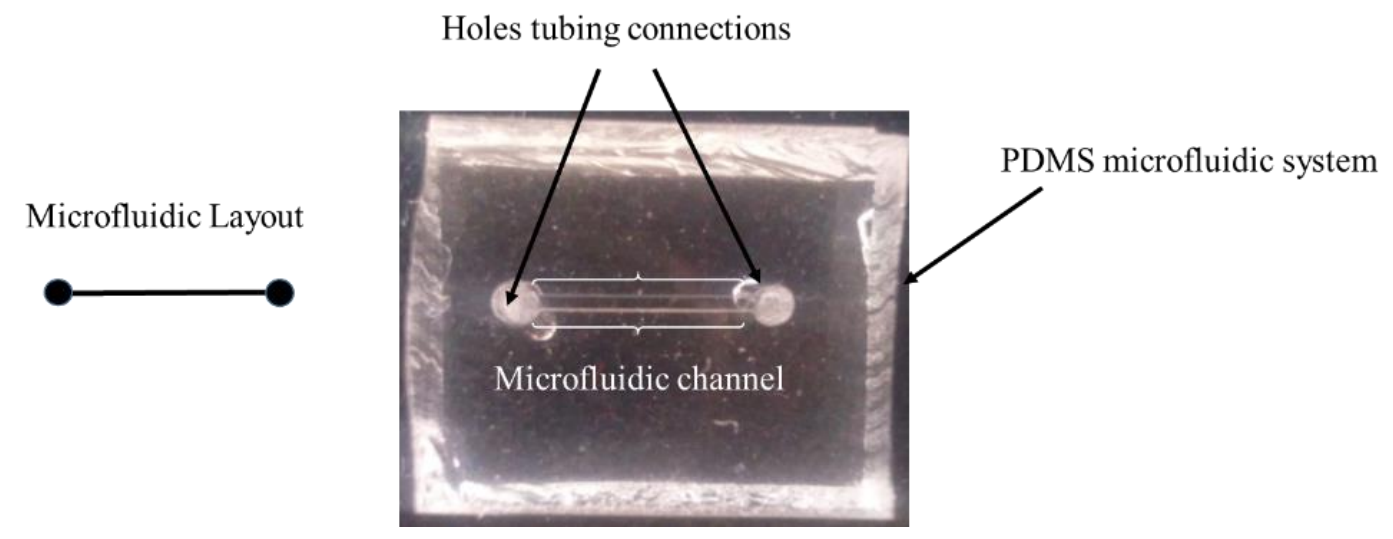

Figure 3.13. (Left): Microfluidic layout. (Right): The microfluidic system with the channel.

With the aim of placing the PDMS microfluidic system on the photonic chip accurately aligned upon the sensing structures, a flip chip machine (Fine-tech) with a tolerance of alignment of only few microns was used to exactly positioning the PDMS on the photonic structures integrated on the SOI chip and align the microfluidic channels with the series of the sensing structures, as can be seen in Fig. 3.14. Once the microfluidic channel is aligned with the sensing structures, a certain pressure was required to the PDMS to adhere it to the chip. 


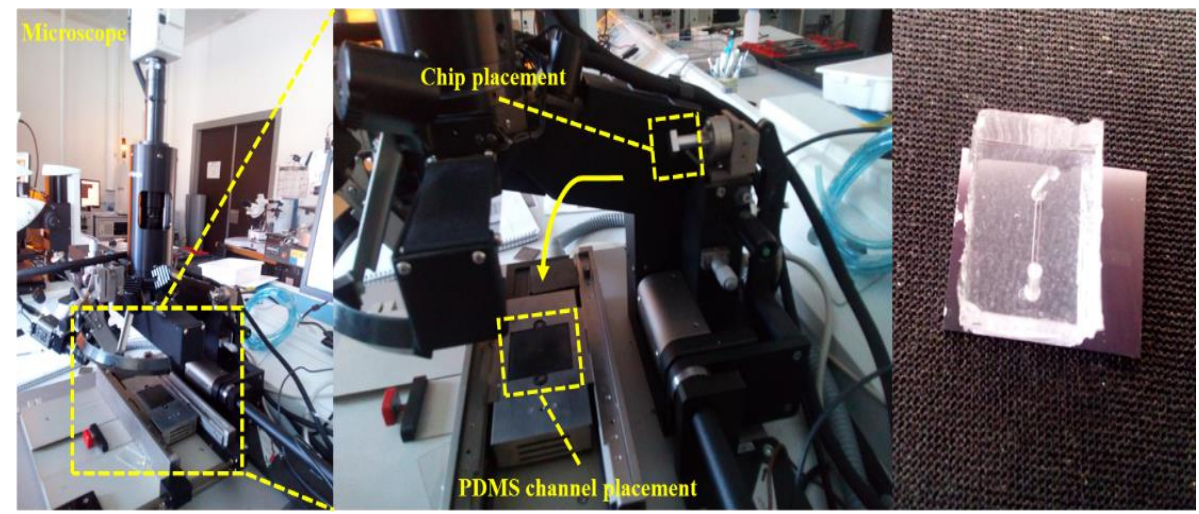

Figure 3.14. Representation of the inverted flip chip microfluidic system positioning.

Figure 3.15 depicts the experimental optofluidic setup used to monitor in real-time the response of the photonic sensing structures during the implementation of the biofunctionalization process. Once the SOI photonic chip is silanized to obtain a vinyl-terminated layer on its surface, it is assembled with the PDMS microfluidic flow cell $((400 \mu \mathrm{m} \times 50 \mu \mathrm{m} \times 6 \mathrm{~mm}$ (width $\times$ height $\times$ length $))$ channel size, which is accessed via 2 polytetrafluoroethylene (PTFE) tubes. The assembled photonic with the fluidic chip is placed on the sample holder of the experimental setup and fixed using a polymethyl methacrylate (PMMA) lid. PDMS and PMMA have been used for the realization of the flow cell and the lid respectively, as they are transparent to UV light and allow to irradiate the photonic chip during the characterization of the photocatalyzed biofunctionalization process.

To characterize the photonic chip in our setup, light from a continuous sweep tunable laser is coupled to the input grating couplers using a fiber collimator. Light coming out from the output grating couplers is measured using an infrared (IR) camera synchronized with the tunable laser in order to obtain the spectra of all the PBG sensing structures within the chip simultaneously (Ruiz-Tórtola et al., 2018). The target solutions were flowed using a syringe pump in withdraw mode and set to a constant flow rate of $10 \mu \mathrm{L} / \mathrm{min}$ which was implemented after experimental optimizations, being notably lower than the calculated maximal flowrate to ensure laminar flow for the selected channel dimensions ( $25 \mu \mathrm{L} / \mathrm{min})$. 

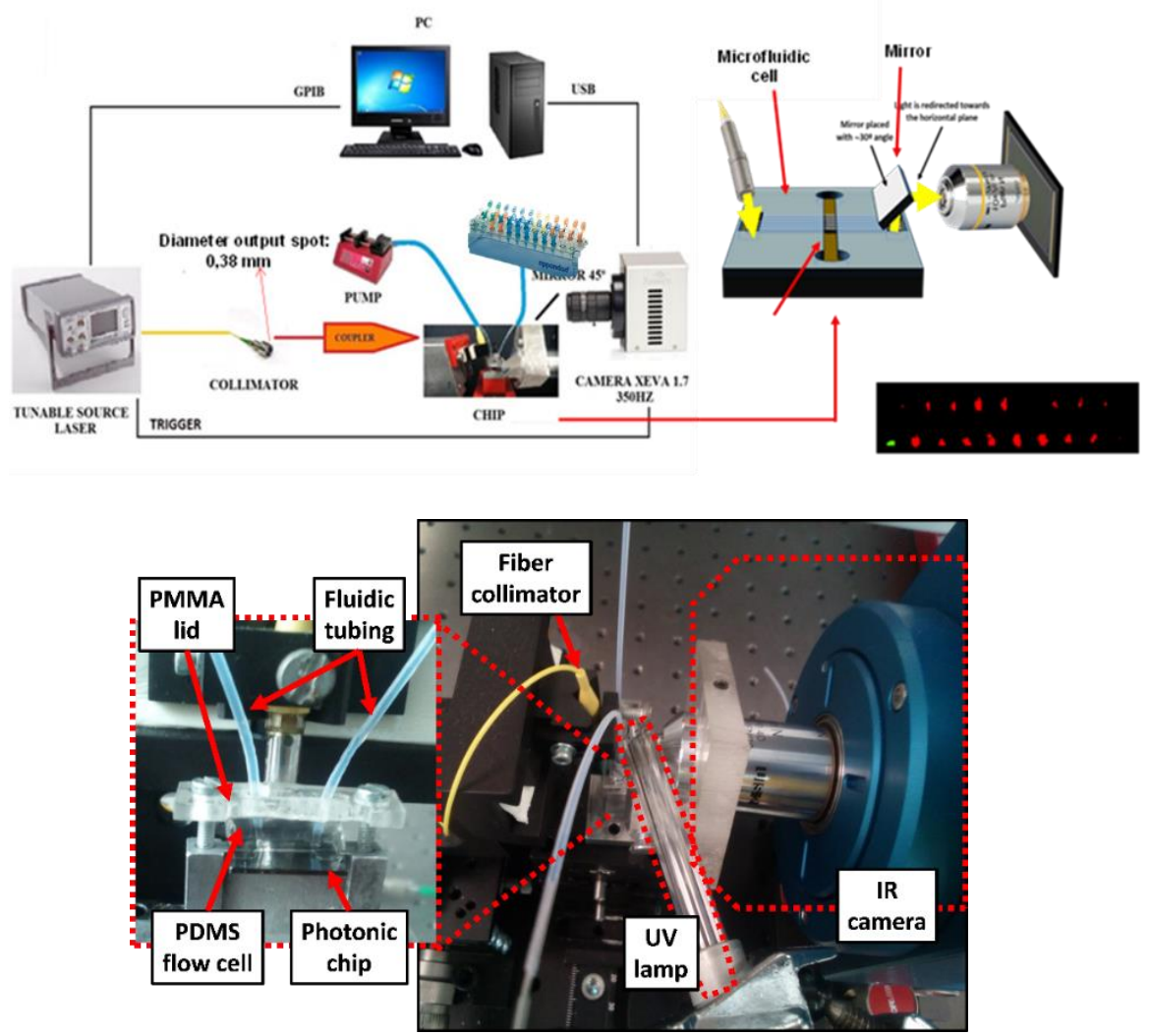

Figure 3.15. (Top) Schematic representation of the measurement set-up. (Bottom right) Experimental setup employed for the interrogation of the photonic chip containing the PBG sensing structures. (Bottom left) Zoomed image that depicts the assembled opto-fluidic system with the chip.

Once the opto-fluidic system was optimized, the next step is transferring the biofunctionalization assay from microarray format to an in-flow mode over the PBG sensing structures to monitor the light-assisted biofunctionalization process in real-time. Figure 3.16 shows the initial spectra in phosphate buffered saline (PBS) $1 \times$ of the photonic sensing structures from one sensor group having their PBG located within the measurement range. It is noteworthy to comment that the PBG edge for the $\mathrm{w}_{\mathrm{i}}=140 \mathrm{~nm}$ structure was not observed because it fell outside our measurement wavelength range. We can see that the increase of the width of the transversal elements $\mathrm{w}_{\mathrm{i}}$ produces a shift of the $\mathrm{PBG}$ edge towards longer wavelengths. Here, the position of the lobe appearing at the PBG edge will be tracked to monitor the spectral shift when the biofunctionalization events take place on the surface of the sensors. 


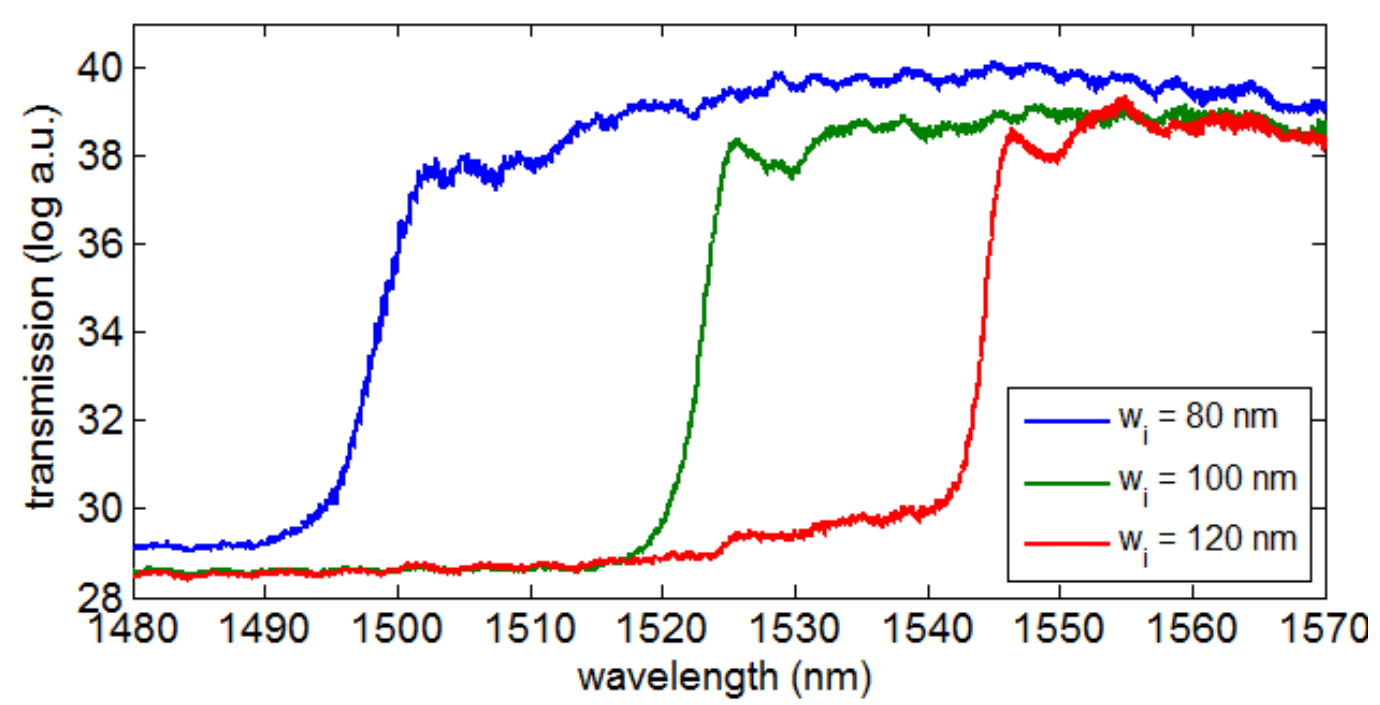

Figure 3.16. Initial spectrum in phosphate saline buffer (PBS) $1 \times$ for the PBG sensing structures within one sensors group (transmission units are given by the analog-to-digital converter (ADC) values provided by the IR camera). Note that the spectrum for the PBG sensing structure with wi $=140 \mathrm{~nm}$ is not depicted because its PBG edge is above the measurement wavelength range.

\subsection{Real-time monitoring of the hIgG immobilization}

Figure 3.17 shows the real-time monitoring results obtained for the UV lightassisted immobilization of half-antibodies over the photonic chip. The sensing response from those $\mathrm{PBG}$ sensing structures having a width of the transversal elements of $\mathrm{w}_{\mathrm{i}}=120 \mathrm{~nm}$ for the 4 sensor groups is depicted. Initially, PBS $1 \mathrm{x}$ is flowed over the vinyl-terminated photonic chip to obtain the initial baseline. Then, the solution containing the haBSA $(20 \mu \mathrm{g} / \mathrm{mL}$ in PBS $1 \times)$ is flowed. As it can be observed in figure 3.18, no photonic sensing response is obtained at that moment for any of the PBG sensing structures despite both the vinyl groups of the SOI surface and the thiol moieties of the haBSA are present. It is not until the photonic chip is irradiated with UV light (at $254 \mathrm{~nm}$ with a power of $6 \mathrm{~mW} / \mathrm{cm}^{2}$ ) and the vinyl-thiol reaction is photocatalyzed and hence the haBSA are immobilized upon the sensor surface, which is translated into a shift of the PBG position. Finally, PBS 
$1 \times$ buffer is flowed again to determine the net spectral shift and to remove any excess of the non-bounded haBSA.

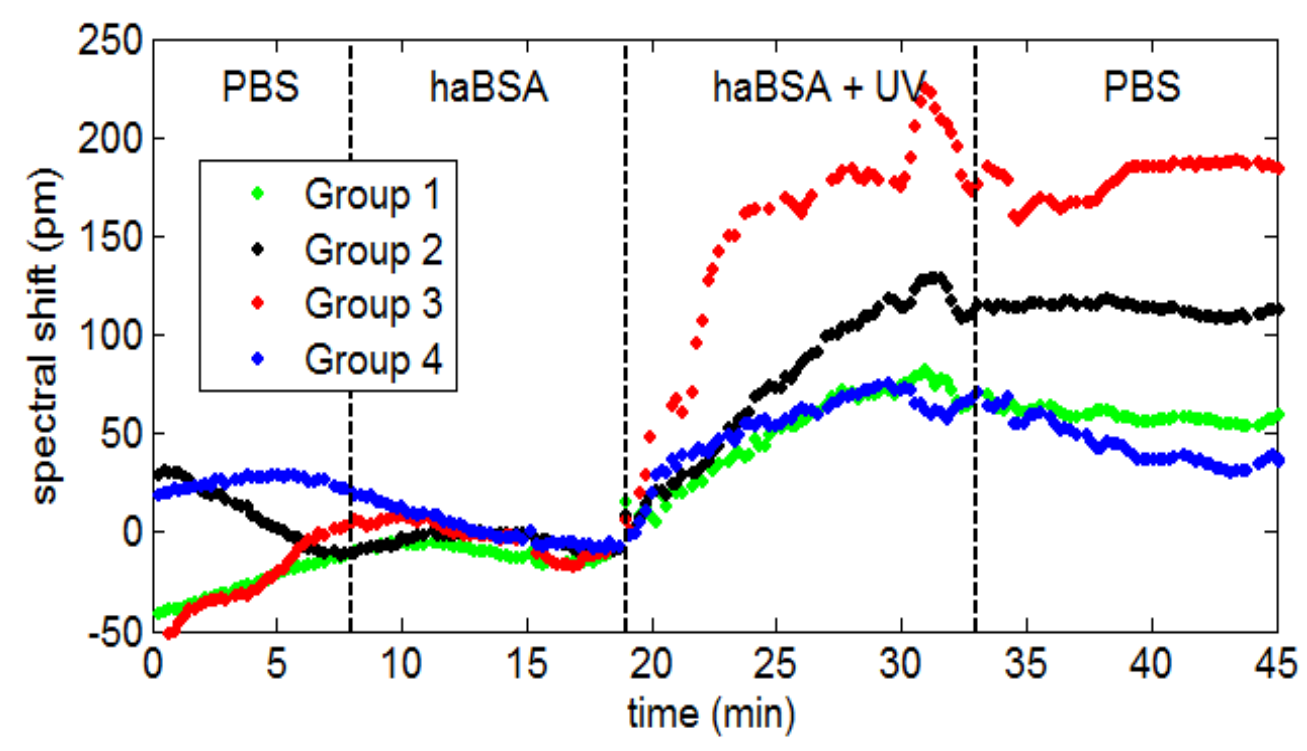

Figure 3.17. Temporal evolution of the PBG spectral shift during the real-time monitoring of the UV light-assisted immobilization of the thiol-terminated haBSA over the vinyl-terminated SOI surface. haBSA are injected at minute 8 and the UV light source is switched on at minute 19.

We can observe that the sensing response is different for each PBG sensing structure belonging to a different sensors group, being higher for those sensor groups placed in a central location of the chip (groups 2 and 3). This is related with the fact that a perfectly homogeneous illumination of the whole photonic chip surface has not been possible due to the presence of the PDMS flow cell, the PMMA lid, and the PTFE tubing on top of the photonic chip, as well as by the limited space to place the UV lamp in the experimental set-up (see figure 3.16). In this context, a better illumination of the central PBG sensors (groups 2 and 3) is produced, which is translated into a higher haBSA immobilization efficiency for those sensors, thus providing a higher spectral shift.

It is also noteworthy to mention that after this biofunctionalization step, $1 \mu \mathrm{g} / \mathrm{mL}$ BSA in PBS $1 \mathrm{x}$ was flowed in order to obtain a certain detection. The results showed a very small PBG shift. From here, an optimization of the biofunctionalization process is required. 


\subsection{Conclusions}

In this chapter, a UV light-assisted biofunctionalization protocol for the immobilization of thiol-terminated bioreceptors onto vinyl-terminated siliconbased sensors was implemented. The fact that UV light is required to induce the immobilization allows to only biofunctionalize those specific positions where the sensing structures are placed, which offers a new way to obtaining higher density of nanoscale biosensing structures being biofunctionalized with different bioreceptors for a large-scale multiplexing level.

Also, we have been able to monitor this photocatalytic immobilization in realtime using an integrated nanophotonic sensing chip, showing that vinyl groups in the surface and thiol groups from the bioreceptors do not react until UV light is present. This result shows the feasibility of using nanophotonic sensors as a tool to study the mechanisms of photo-induced reactions in real-time. Additionally, this is, to our knowledge, the first time that photonic sensing structures are biofunctionalized with half antibodies, which can also mean an advantage in terms of higher surface coverage density, lower thickness of the recognition layer, and proper orientation of the antibodies binding sites and hence the enhancement of the sensitivity of the photonic sensors.

\subsection{References}

Alonso, R., Jiménez-Meneses, P., García-Rupérez, J., Bañuls, M.-J., Maquieira, Á., 2018. Thiol-ene click chemistry towards easy microarraying of halfantibodies. Chem. Commun. 54, 6144-6147. https://doi.org/10.1039/C8CC01369A.

Caroselli, R., García Castelló, J., Escorihuela, J., Bañuls, M.J., Maquieira, Á., García-Rupérez, J., 2018. Experimental Study of the Oriented Immobilization of Antibodies on Photonic Sensing Structures by Using Protein A as an Intermediate Layer. Sensors 18, 1012. https://doi.org/10.3390/s18041012.

Chin, C.D., Linder, V., Sia, S.K., 2007. Lab-on-a-chip devices for global health: 
Past studies and future opportunities. Lab Chip 7, 41-57. https://doi.org/10.1039/B611455E.

Cline, D.J., Redding, S.E., Brohawn, S.G., Psathas, J.N., Schneider, J.P., Thorpe, C., 2004. New Water-Soluble Phosphines as Reductants of Peptide and Protein Disulfide Bonds: Reactivity and Membrane Permeability ${ }^{\dagger}$. Biochemistry 43, 15195-15203. https://doi.org/10.1021/bi048329a.

Escorihuela, J., Bañuls, M.-J., Grijalvo, S., Eritja, R., Puchades, R., Maquieira, Á., 2014. Direct Covalent Attachment of DNA Microarrays by Rapid Thiol-Ene "Click" Chemistry. Bioconjug. Chem. 25, 618-627. https://doi.org/10.1021/bc500033d.

Escorihuela, J., Bañuls, M.J., Puchades, R., Maquieira, Á., 2012. DNA microarrays on silicon surfaces through thiol-ene chemistry. Chem. Commun. 48, 2116. https://doi.org/10.1039/c2cc17321b.

Estevez, M.C., Alvarez, M., Lechuga, L.M., 2012. Integrated optical devices for lab-on-a-chip biosensing applications. Laser Photon. Rev. 6, 463-487. https://doi.org/10.1002/lpor.201100025.

González-Guerrero, A.B., Alvarez, M., Castaño, A.G., Domínguez, C., Lechuga, L.M., 2013. A comparative study of in-flow and micro-patterning biofunctionalization protocols for nanophotonic silicon-based biosensors. J. Colloid Interface Sci. 393, 402-410. https://doi.org/10.1016/J.JCIS.2012.10.040.

González-Lucas, D., Bañuls, M.-J., García-Rupérez, J., Maquieira, Á., 2017. Covalent attachment of biotinylated molecular beacons via thiol-ene coupling. A study on conformational changes upon hybridization and streptavidin binding. Microchim. Acta 184, 3231-3238. https://doi.org/10.1007/s00604017-2310-4.

Johnson, B.N., Mutharasan, R., 2012. Biosensing using dynamic-mode cantilever sensors: A review. Biosens. Bioelectron. 32, 1-18. https://doi.org/10.1016/J.BIOS.2011.10.054. 
Jones, W.P., Launder, B.E., 1973. The calculation of low-Reynolds-number phenomena with a two-equation model of turbulence. Int. J. Heat Mass Transf. 16, 1119-1130. https://doi.org/10.1016/0017-9310(73)90125-7.

Jonkheijm, P., Weinrich, D., Schröder, H., Niemeyer, C.M., Waldmann, H., 2008. Chemical Strategies for Generating Protein Biochips. Angew. Chemie Int. Ed. 47, 9618-9647. https://doi.org/10.1002/anie.200801711.

Kolb, H.C., Finn, M.G., Sharpless, K.B., 2001. Click Chemistry: Diverse Chemical Function from a Few Good Reactions. Angew. Chem. Int. Ed. Engl. 40, 20042021.

Makaraviciute, A., Jackson, C.D., Millner, P.A., Ramanaviciene, A., 2016. Considerations in producing preferentially reduced half-antibody fragments. J. Immunol. Methods 429, 50-56. https://doi.org/10.1016/j.jim.2016.01.001.

Phaner-Goutorbe, M., Dugas, V., Chevolot, Y., Souteyrand, E., 2011. Silanization of silica and glass slides for DNA microarrays by impregnation and gas phase protocols: A comparative study. Mater. Sci. Eng. C 31, 384-390. https://doi.org/10.1016/J.MSEC.2010.10.016.

Povinelli, M.L., Johnson, S.G., Joannopoulos, J.D., 2005. Slow-light, band-edge waveguides for tunable time delays. Opt. Express 13, 7145. https://doi.org/10.1364/OPEX.13.007145.

Ruiz-Tórtola, Á., Prats-Quílez, F., González-Lucas, D., Bañuls, M.-J., Maquieira, Á., Wheeler, G., Dalmay, T., Griol, A., Hurtado, J., Bohlmann, H., Götzen, R., García-Rupérez, J., 2018. Experimental study of the evanescent-wave photonic sensors response in presence of molecular beacon conformational $\begin{array}{llll}\text { changes. } & \text { J. } & \text { Biophotonics } & \text { e201800030. }\end{array}$ https://doi.org/10.1002/jbio.201800030.

Sandeep Arya, Saleem Khan, Akhil Vaid, Harneet Kour, P.L. (University of J., 2014. Microfluidic Mechanics and Applications: a Review. J. Nano- Electron. Phys. 5, 1-12. ttps://doi.org/10.3390/s1712293.

Sato, K., Mawatari, K., Kitamori, T., 2008. Microchip-based cell analysis and 
clinical diagnosis system. https://doi.org/10.1039/b814098g.

Vestergaard, M., Kerman, K., Tamiya, E., Vestergaard, M., Kerman, K., Tamiya, E., 2007. An Overview of Label-free Electrochemical Protein Sensors. Sensors 7, 3442-3458. https://doi.org/10.3390/s7123442.

Wu, J., Dong, M., Santos, S., Rigatto, C., Liu, Y., Lin, F., Wu, J., Dong, M., Santos, S., Rigatto, C., Liu, Y., Lin, F., 2017. Lab-on-a-Chip Platforms for Detection of Cardiovascular Disease and Cancer Biomarkers. Sensors 17, 2934. https://doi.org/10.3390/s17122934. 


\section{Chapter 4}

\section{BSA and CVD}

\section{biomarkers detection}

\section{by PBG biosensors}

\subsection{Introduction}

In this chapter, we present the experimental work carried out to characterize and enhance the interaction with the target analytes in evanescence wave-based sensors, specifically for the case of PBG sensing structures, and thus to increase their sensitivity. After designing and fabricating the PBG sensing structures, its evanescent field has been thoroughly characterized using Scanning Near Field Optical Microscopy (SNOM) to determine how the interaction will vary with the distance to the sensor surface. Then, the PBG structure has been biofunctionalized to use it for biosensing purposes. Here, the biofunctionalization protocol was modified considering that the direct in-flow biofunctionalization presented in the previous chapter gave very low detection results. Therefore, the UV-induced immobilization of the half antibodies was carried out by spotting, what may enhance the surface concentration of the bioreceptor over the PBG sensors, since the UV lamp alignment, space and the tubing issues were avoided, which may lead to an enhancement of the detection signal. 
PBG biosensing experiment has been first launched for Bovine Serum Albumin (BSA) detection previously to the CVD biomarkers detection assays with the aim of the system optimization. A global scheme of whole experiments is described in the next figure.

1)

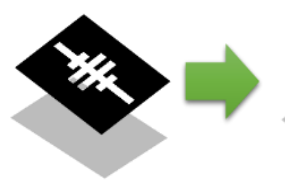

Nanofabrication
2)

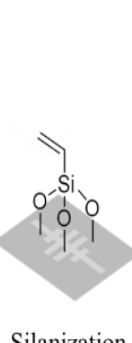

3)

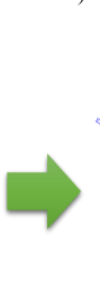

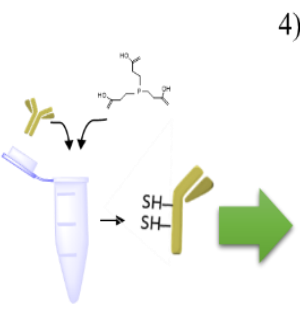

aBSA cleveage
4)

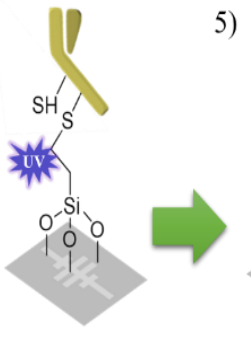

Biofunctionalization

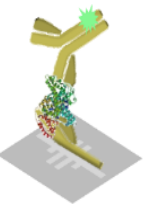

Biodetection

Figure 4.1. Brief scheme of the whole protocol.

Finally, CVD biomarkers such as CRP, Mb, cTnI and cTnT were detected at the required concentrations by modifying when needed, the recognition layer over the PBG sensing surface.

\subsection{Scanning Near Field Optical Microscope (SNOM) characterization}

Given the importance of the evanescent field profile over the sensing performance, the near-field behavior of the PBG sensing structures has been studied using SNOM technology, which has been used in previous works to characterize SOI structures as nanoatennas (Díaz-Fernández et al., 2018; Espinosa-Soria et al., 2018). SNOM measurements were performed with a tailored MultiView 4000 system (Nanonics Imaging Ltd.) working in collection mode as schematized below (see figure 4.2). A cleaved single mode optical fiber was used to couple light from a tunable laser (Keysight $81980 \mathrm{~A})$ to the photonic structures on the chip via their input grating couplers. A bent fiber tip with a $500 \mathrm{~nm}$ aperture, $\mathrm{Cr} / \mathrm{Au}$ coated, pre-mounted on a tuning-fork working in tapping mode at $36.19 \mathrm{kHz}$ was used to scan the photonic structures and measure the near-field signal using a FWPR-S Femtowatt 
Photoreceiver. This tip was placed perpendicular to the sample, allowing a mode matching between the tip and the measured structures (Bazylewski et al., 2017; Dvořák et al., 2017). The whole system (photonic chip, input fiber and SNOM tip) can be previsualized with an optical microscope that enables a correct input fiberchip alignment and an accurate positioning of the SNOM probe on top of the PBG structure.

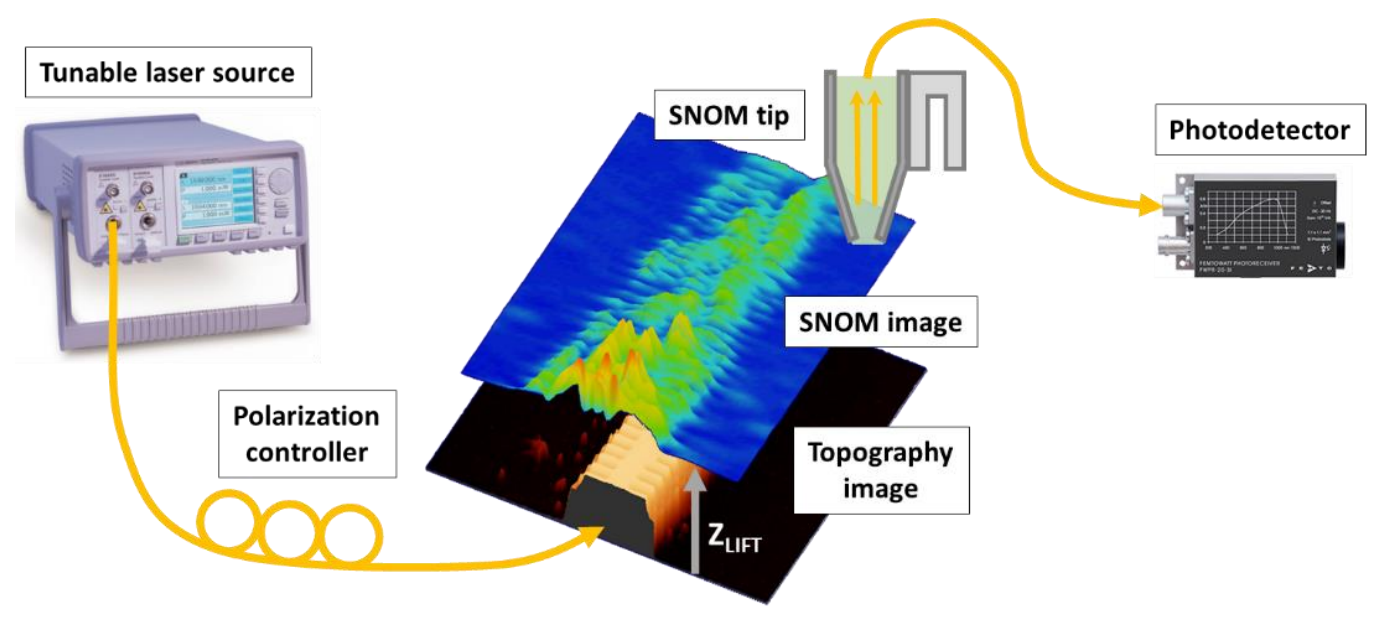

Figure 4.2. Schematic view of the SNOM characterization set-up.

Figure 4.3 shows the SNOM signal measured for one of the PBG sensing structures having the transversal elements of width $\mathrm{w}_{\mathrm{i}}=120 \mathrm{~nm}$ for different excitation wavelengths and for an air upper cladding. Excitation wavelengths between 1530 $\mathrm{nm}$ and $1580 \mathrm{~nm}$ have been considered in the measurements. SNOM images obtained for wavelengths $1530 \mathrm{~nm}$ and $1540 \mathrm{~nm}$ show a high back-scattering signal at the input of the PBG structure and no transmission was observed through the structures, thus confirming the presence of the PBG region. For wavelengths from $1550 \mathrm{~nm}$ to $1580 \mathrm{~nm}$, transmission over the whole PBG structure can be observed, thus indicating that the Bloch modes of the structure are being excited and that we are out from the PBG. Note that some backscattering is observed at the access of the PBG structure for all the excitation wavelengths except for $1570 \mathrm{~nm}$, indicating that an optimal coupling is produced for this wavelength. Note that the PBG edge position determined for SNOM measurements is around $1550 \mathrm{~nm}$, while the PWE and FDTD simulations predicted a PBG edge located around $1530 \mathrm{~nm}$. This 
difference can be attributed to slight variations in the dimensions of the fabricated structure or to the presence of $\mathrm{SiO}_{2}$ residues when opening the channel over the PBG sensing structures.
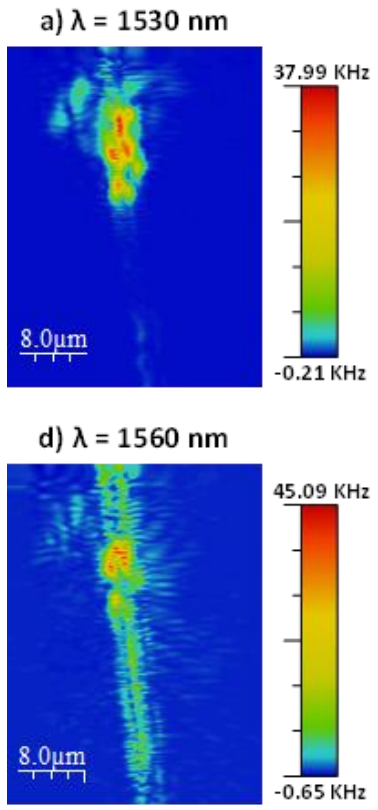

b) $\lambda=1540 \mathrm{~nm}$

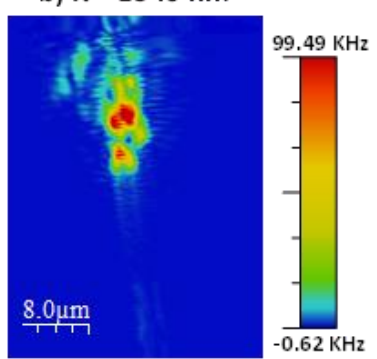

e) $\lambda=1570 \mathrm{~nm}$

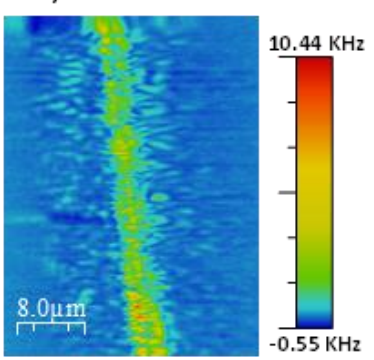

c) $\lambda=1550 \mathrm{~nm}$

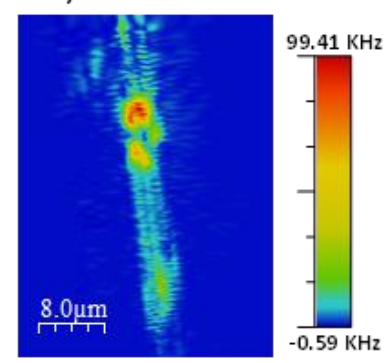

f) $\lambda=1580 \mathrm{~nm}$

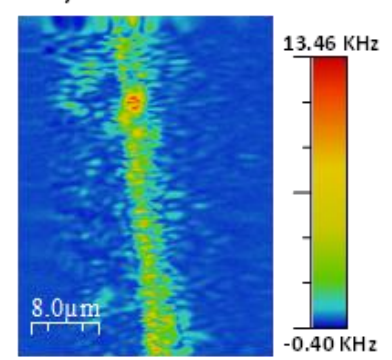

Figure 4.3. SNOM measurements obtained at different wavelengths (from 1530 $\mathrm{nm}$ to $1580 \mathrm{~nm}$ ) for a PBG sensing structure having transversal element of width $\mathrm{w}_{\mathrm{i}}=120 \mathrm{~nm}$. The measurements are done at $20 \mathrm{~nm}$ from the PBG structure surface.

Once the near field behavior of the PBG sensing structure was characterized depending on the operation wavelength (i.e., inside or outside the PBG), the next step was measuring the decay length of the evanescent field to characterize how the sensing interaction will vary with the height. Figure 4.4 (a) shows the SNOM measurements performed at different distances from the surface of the structure for an excitation wavelength of $1570 \mathrm{~nm}$ (which provided the best coupling to the PBG structure). These SNOM images show how the intensity of the evanescent field significantly decreases for heights above $100 \mathrm{~nm}$.

This SNOM characterization was performed in parallel to a simulation study in order to compare the evanescent wave behavior experimentally and computationally. To this aim, FDTD simulations were done using CST Microwave 
Studio, which is specially created to solve electromagnetic problems in three dimensions where the working frequencies are high, as it is the case that concerns us. For our simulations, a 61 period PBG structure has been considered, such as for the one manufactured (Espinosa-Soria et al., 2018). To perform the system of equations that the simulation resolves, a hexahedral type mesh has been performed with 1.34 million cells, with at least 4 divisions per wavelength. The FDTD propagation simulations were carried out and the evanescent field intensity was measured for the same heights used in the SNOM measurements, as shown in figure 4.4 (b). Note that as a shift of about $\sim 20 \mathrm{~nm}$ of the PBG edge position was observed between the experimental characterization and the simulations, FDTD propagation results have been obtained using an excitation wavelength of $1550 \mathrm{~nm}$ to consider the same scenario than in the SNOM characterization. Both for the SNOM measurements shown in figure 4.4 (a) and for the FDTD simulations shown in figure 4.4 (b), maximum values of the evanescent field intensity over different periods of the PBG structure were obtained and represented in figure 4.4 (c) as a function height. As it can be observed in the graph, a strong exponential decrease of the evanescent field intensity is observed, thus confirming the need of performing the biodetection as close to the sensing surface as possible for a higher sensitivity. Note also the almost perfect agreement between the experimental SNOM characterization and the FDTD simulations. 
a)

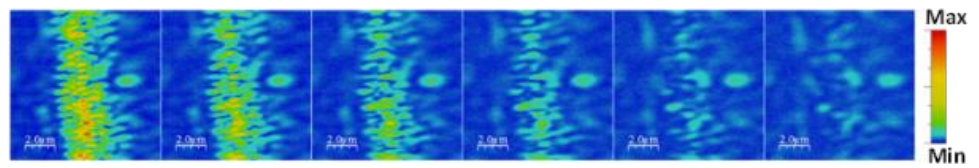

$Z_{\mathrm{UFT}}=0 \mathrm{~nm} \quad Z_{\mathrm{UFT}}=50 \mathrm{~nm} \quad Z_{\mathrm{UFT}}=100 \mathrm{~nm} \quad Z_{\mathrm{UFT}}=150 \mathrm{~nm} \quad Z_{\mathrm{UFT}}=250 \mathrm{~nm} \quad Z_{\mathrm{UFT}}=350 \mathrm{~nm}$

b)

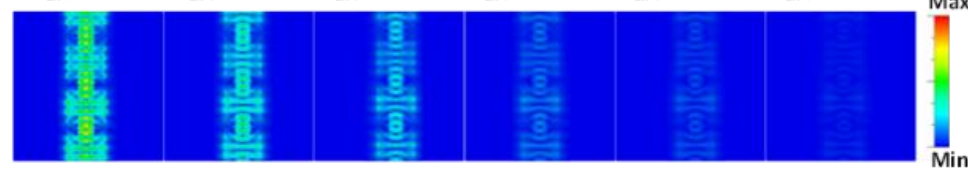

c)

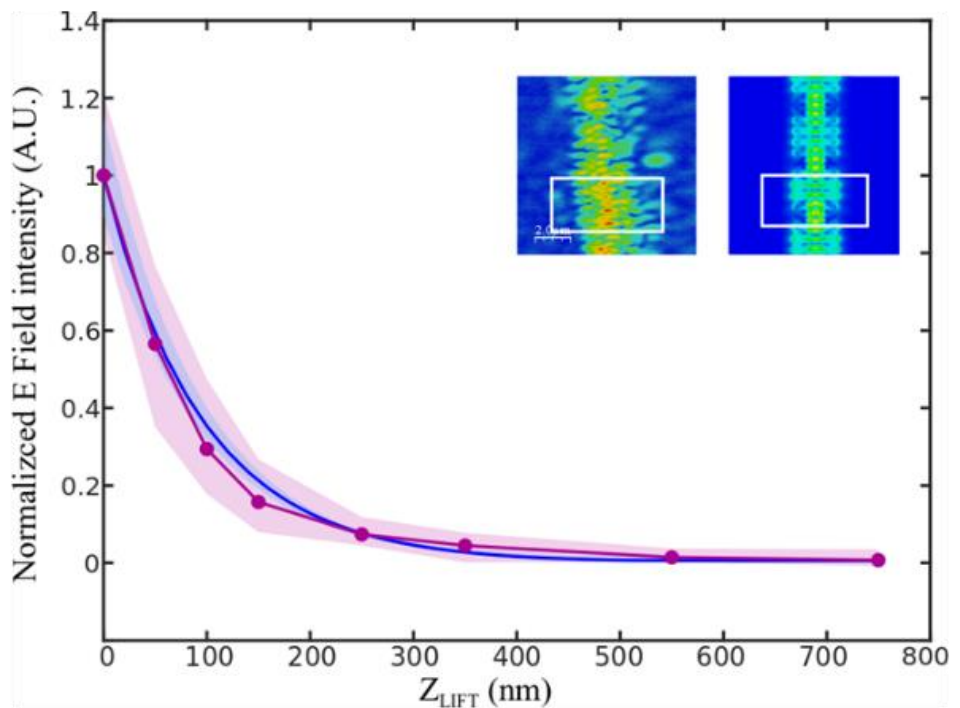

Figure 4.4. (a) SNOM measured and (b) FDTD simulated near-field at different vertical distances $\left(\mathrm{Z}_{\mathrm{LIFT}}\right)$ from the surface of the PBG sensing structure. An excitation wavelength of $1570 \mathrm{~nm}$ is used for the SNOM measurements, while a wavelength of $1550 \mathrm{~nm}$ has been used in the FDTD simulations. (c) Normalized intensity of the evanescent field as a function of the distance to the sensor surface for the SNOM measurements (purple color) and for the FDTD simulations (blue color). The shaded areas represent the standard deviation of the raw SNOM measurements and the FDTD simulations along the area of the

PBG structure highlighted in the insets.

Since SNOM setup does not allow to perform near-field measurements of the photonic structures when having a water upper cladding and considering the good agreement between simulations and experiments previously shown, we have used FDTD simulations to determine the evanescent field profile in that scenario. Figure 4.5 shows the electric field profile simulated when considering air and water upper cladding, as well as the variation of its intensity as a function of the distance to the sensor surface for these two scenarios. Note that, to consider an equivalent situation, 
both simulations have been made considering an excitation wavelength located at 20 nm from the selected PBG edge (i.e., $1550 \mathrm{~nm}$ for an air upper cladding and $1600 \mathrm{~nm}$ for a water upper cladding). As it can be observed, the decay profile of the evanescent field in air and in water are in match.
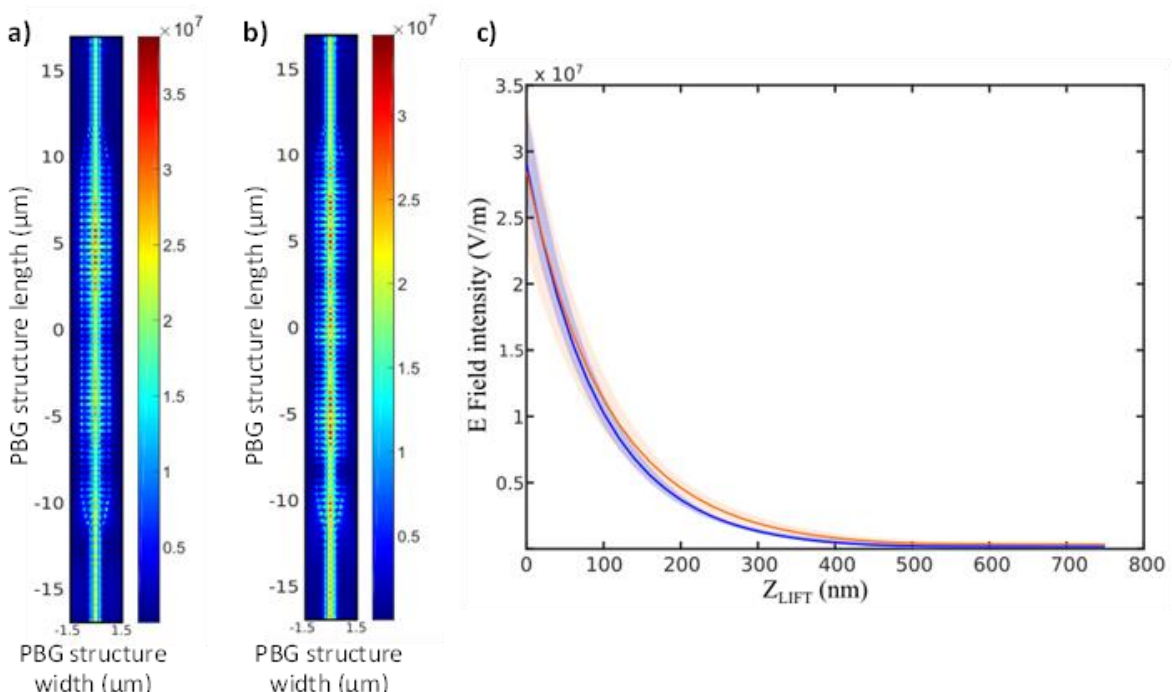

Figure 4.5. (a, b) FDTD simulated electric near-field intensity (V/m) through the PBG structure for air $(\lambda=1550 \mathrm{~nm})$ and water upper cladding $(\lambda=1600$ $\mathrm{nm}$ ), respectively. (c) Variation of the electric field intensity as a function of the distance to the sensor surface for the FDTD simulations when considering an air (blue color) and a water upper cladding (red color). The shaded areas represent the standard deviation of the intensity along the PBG structure.

\subsection{Drop-casting half antibody biofunctionalization}

As determined from the evanescent field characterization by means of SNOM measurements and FDTD simulations, a very thin biorecognition layer is required for an optimum interaction of the biosensor with the target analytes. In order to achieve that thin biorecognition layer, we have developed the light-assisted biofunctionalization approach that allows the covalent immobilization of thiolterminated bioreceptors onto a vinyl-terminated surface as explained in the previous chapter. But in this case, since the in-flow biofunctionalization strategy previously used in chapter 3 gave very low detection signal in the following biosensing assay, 
now we have immobilized the hIgG by spotting it over the sensing structures as depicted in figure 4.6. Here, $2 \mu \mathrm{L}$ of $20 \mu \mathrm{g} / \mathrm{mL}$ rabbit half BSA antibodies have been drop casted over the sensing area and irradiated for 5s using the UV lamp (254 $\mathrm{nm}$ with $50 \mathrm{~mW} / \mathrm{cm}^{2}$ ). Considering the thickness of the initial organosilane layer $(\sim 4.5 \mathrm{~nm})$ as obtained from ellipsometry measurements, and by estimating the thickness of the half antibodies deposited on the surface $(\sim 2.5 \mathrm{~nm})$, we determine that a total thickness of the whole biorecognition layer of only $\sim 7 \mathrm{~nm}$ was achieved. When determining the immobilization density by fluorescence microarray format, densities corresponding to a close-packed monolayer of the half antibodies were obtained with standard deviation of $8 \%$ indicating a good reproducibility in the immobilization method.

a)

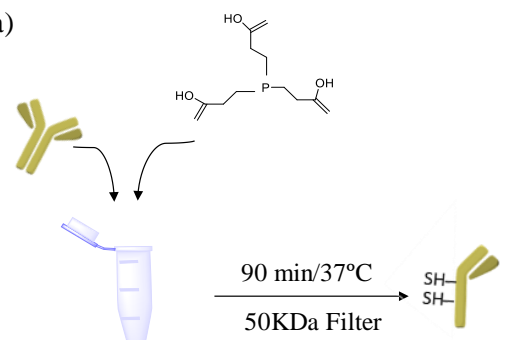

b)

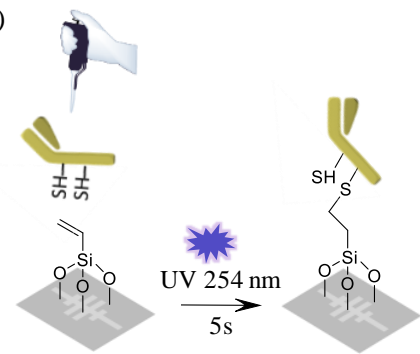

Figure 4.6. (a) haBSA cleavage protocol. (b) Light-assisted immobilization of the thiol-terminated haBSA onto the vinyl-terminated surface of the PBG sensing structures by spotting.

\subsection{Direct and real-time BSA detection}

For the BSA real-time detection, the employed measurement set-up and the tracking process is identical to that previously described in chapter 3 .

Before performing the BSA detection experiment, a bulk sensitivity test was carried out. The sensitivity experiment consists basically on the flow of pure Deionized Water (DIW) followed by a mixture of DIW and 5\% of ethanol (EtOH) and back to pure DIW. This experiment is performed to determine the bulk sensitivity of our different $\mathrm{w}_{\mathrm{i}}$ sensing structures to changes of the refractive index (RI) of the cladding. Results are depicted in figure 4.7. 


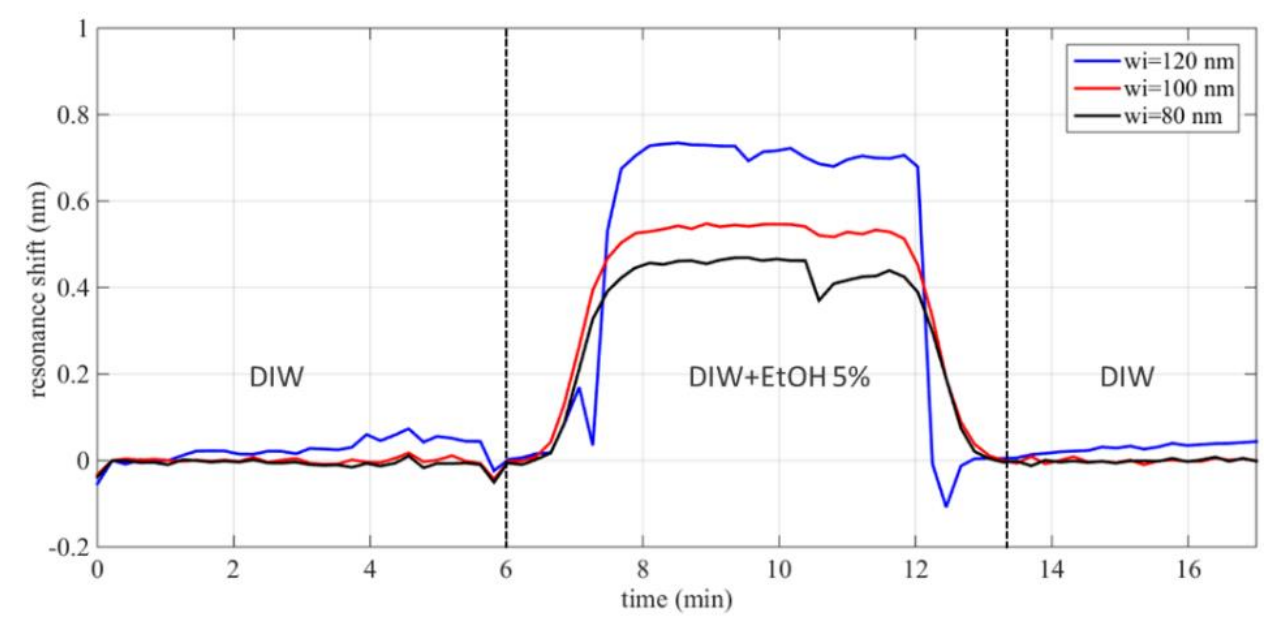

Figure 4.7. Sensitivity test carried out for different $\mathrm{w}_{\mathrm{i}}$ sensing structures.

As previously described in (García-Rupérez et al., 2010), the refractive index of DIW and EtOH dilutions for ethanol proportions below $6 \%$ in volume is linearly approximated as:

$$
n_{\text {dilution }}=1.024 \times(\% / 100) \times n_{E t O H}+1 \times(1-\% / 100) \times n_{D I W} .
$$

Equation 4.1

From here, we determine that the RI of the DIW-EtOH dilutions for our working conditions $\left(\lambda \approx 1550 \mathrm{~nm}\right.$ and $\left.\mathrm{T} \approx 25^{\circ} \mathrm{C}\right)$ are $\mathrm{n}_{\text {DIW }}=1.3173$ and $\mathrm{n}_{\text {DIW-EtOH } 5 \%}=1.3199$. The sensitivity equation is represented as following:

$$
S(n m / R I U)=\frac{\Delta \lambda(\mathrm{nm})}{\Delta n(\mathrm{RIU})}
$$

where $S$ is the sensitivity, $\Delta \lambda$ is the measured wavelength shift and $\Delta \mathrm{n}$ is the refractive index variation. By using the measured wavelength shifts from figure 4.8 and considering the refractive index variations calculated from equation 4.1, we determine the sensitivities of our sensors as shown in table 4.1.

Table 4.1. Experimental sensitivities of $\mathrm{w}_{\mathrm{i}}=80,100$ and $120 \mathrm{~nm}$ PBG structures in (nm/RIU).

\begin{tabular}{cccc}
\hline & $\mathrm{W}_{\mathrm{i}}=80 \mathrm{~nm}$ & $\mathrm{~W}_{\mathrm{i}}=100 \mathrm{~nm}$ & $\mathrm{~W}_{\mathrm{i}}=120 \mathrm{~nm}$ \\
\hline Sensitivity (nm/RIU) & 165.38 & 215.38 & 292.3 \\
\hline
\end{tabular}


We can observe that a higher sensitivity is obtained when $\mathrm{w}_{\mathrm{i}}$ increases, thus indicating a better interaction of the light with the upper cladding medium.

A blank test was performed to check the detection system availability. First, fluorophore-labelled detection antibody was deposited directly over the SOI surface biofunctionalized with BSA half antibodies without the presence of the target BSA antigen in between, and then it was rinsed with the buffer again to eliminate the BSA antibody excess. No fluorescence was detected, what confirms that the specificity test was successful.

Once the sensitivity and selectivity studies were successful, a direct detection BSA experiment was performed. Figure 4.8 shows the results obtained for one of the groups of PBG sensing structures in the biosensing experiments carried out. After an initial flow of PBS-T buffer (PBS $+0.01 \%$ Tween 20) for 10 minutes to establish the baseline, BSA at $1 \mu \mathrm{g} / \mathrm{mL}$ in PBS-T was flowed over the biofunctionalized PBG sensing structures for 20 minutes for the real-time detection of the target antigens. Then, PBS-T buffer was flowed again for 10 minutes to eliminate BSA excess. Note that PBS-T buffer was employed in these experiments to prevent non-specific interactions and BSA adsorption. As it can be observed in figure 4.9, the target BSA was successfully detected by the PBG sensing structures, obtaining shifts of 50, 95 and $140 \mathrm{pm}$ for structures having $\mathrm{w}_{\mathrm{i}}=80,100$ and $120 \mathrm{~nm}$, respectively. Note that results for the PBG structure having $\mathrm{w}_{\mathrm{i}}=140 \mathrm{~nm}$ have not been included because its PBG edge was out from our measurement range. From the experimental results of the figure 4.7 and figure 4.8 , we can also observe the great influence of the $\mathrm{w}_{\mathrm{i}}$ parameter of the PBG structures over their sensitivities, obtaining an increase of the shift of 45 pm every time that the width parameter $\mathrm{w}_{\mathrm{i}}$ is increased by $20 \mathrm{~nm}$ in the BSA detection experiments. Finally, fluorophore labelled antiBSA at $20 \mu \mathrm{g} / \mathrm{mL}$ was flowed with the aim of the verification of the surface homogeneity by checking the fluorescence intensity. Figure 4.9 shows the high homogeneity and intensity over the whole sensing area confirming the adequate realization of all the steps of functionalization and detection. 


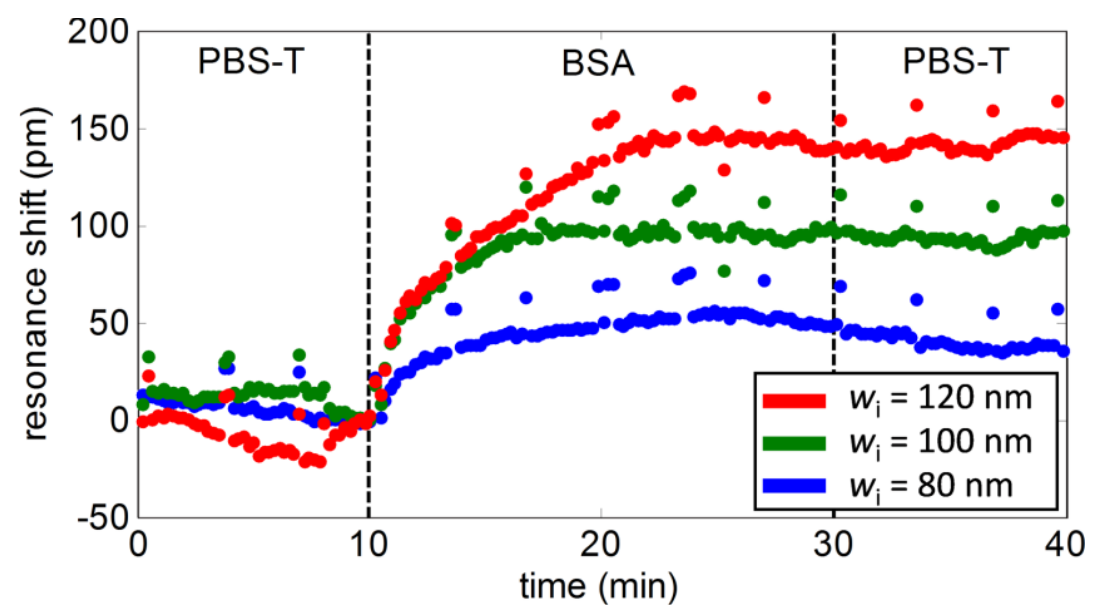

Figure 4.8. Experimental results of the real-time direct detection of BSA. Experimental data corresponding to the different $\mathrm{w}_{\mathrm{i}}$ PBG structures.

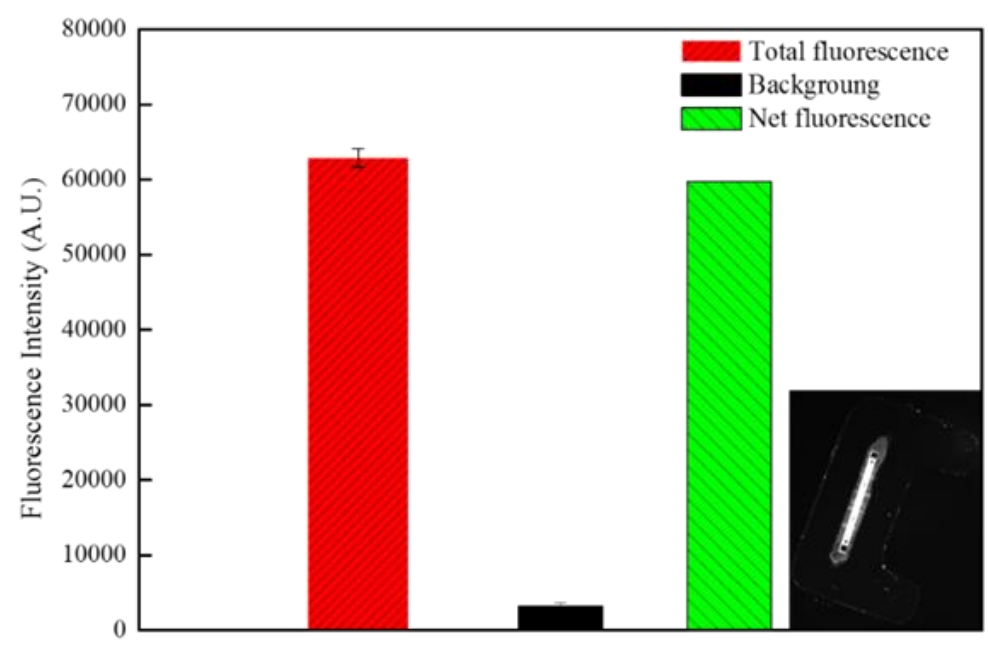

Figure 4.9. Fluorescence intensity test of the photonic chip. (inset) chip image after the flow of the fluorophore-labelled antiBSA.

Once we performed the direct real-time detection of the BSA successfully, the following step is the application of this strategy for the previously mentioned CVD biomarkers detection.

\subsection{Direct and real-time detection of CVD biomarkers}

Previously to the CVD detection performance, we performed a selectivity study in order to confirm the selectivity of the half antibodies. First, CRP half antibodies were drop casted and UV light irradiated to biofunctionalize a flat SOI surface; then fluorophore-labelled BSA was incubated for $30 \mathrm{~min}$ and the fluorescence was 
checked after cleaning by PBST, obtaining no fluorescence signal. No PBG shift was detected, and the fluorescence test gave no fluorescence, so the results confirm the selectivity of the half antibodies.

Once the selectivity was checked and the biofunctionalization protocol was optimized and the detection of BSA was correctly achieved, the next step of CVD biomarkers detection will consist on the biofunctionalization of the sensing surface by means of drop-casting of the specific half antibody of each antigen. Note that for very low CVD biomarkers concentrations cases, a fluorophore-labelled detection antibody is flowed in the end in order to enhance the detection signal (the PBG shift).

\subsubsection{C-Reactive Protein (CRP) detection}

Here, we apply our photonic sensing system based on PBG sensing structures previously described and applied for BSA to detect the CRP. Here, the chemical surface functionalization was carried out using the TEVS $1 \%$ in water carrier and the biofunctionalization protocol carried out was by drop casting the half antibody of CRP (haCRP). Here, the protein concentration to detect is of $250 \mathrm{ng} / \mathrm{mL}$. The other conditions were the same as applied for BSA detection experiment. Results are depicted in figure 4.10 .

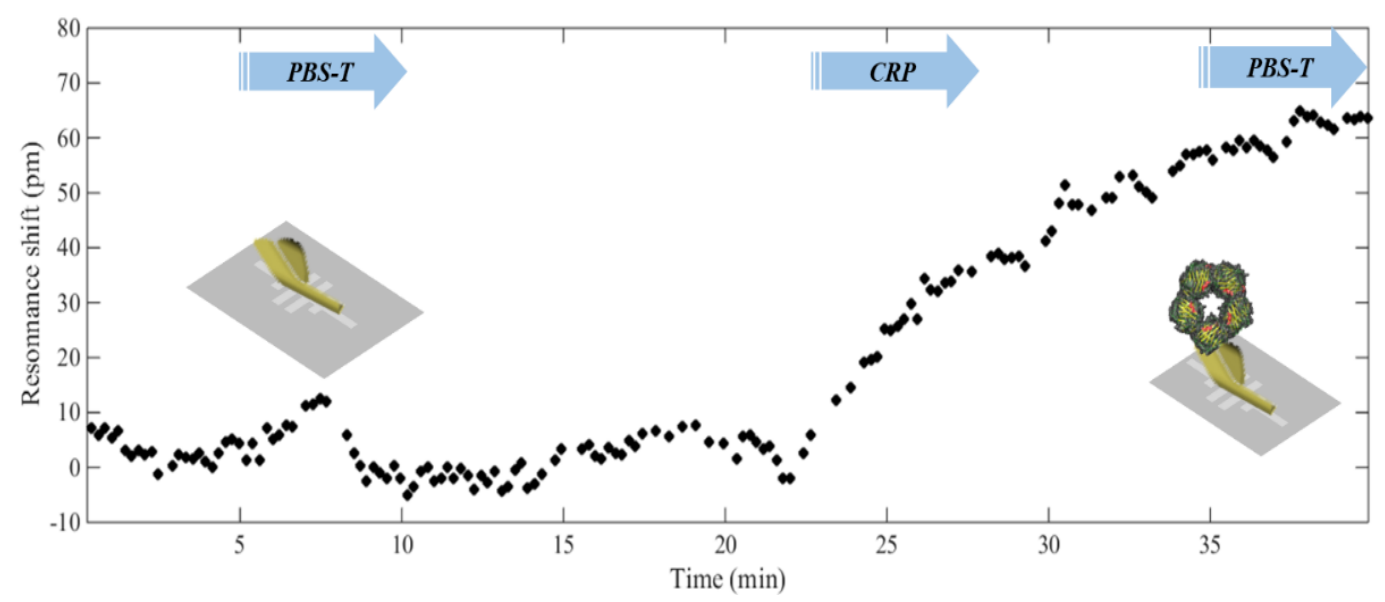

Figure 4.10. $250 \mathrm{ng} / \mathrm{mL}$ CRP direct detection and representative scheme. 
The obtained detection results are for the PBG structure with $\mathrm{w}_{\mathrm{i}}=120 \mathrm{~nm}$. In the biosensing experiments carried out, after an initial flow of PBS-T buffer (PBS + $0.01 \%$ Tween 20) for 20 minutes to establish the baseline (5 minutes might be enough as well), $250 \mathrm{ng} / \mathrm{mL}$ of CRP in PBS-T was flowed over the biofunctionalized PBG sensing structures for 15 minutes for the real-time detection of the target antigens. Then, PBS-T buffer was flowed again to eliminate CRP excess. As it can be observed in figure 4.10, the target CRP was successfully detected by the PBG sensing structure, obtaining a shift of $65 \mathrm{pm}$, in the same order as found by ring resonators for $100 \mathrm{ng} / \mathrm{mL}$ (Luchansky et al., 2011).

After the CRP detection, a more specific biomarker for CVD is a must, since as previously mentioned in chapter $1, \mathrm{CRP}$ is an inflammation marker and predicts cardiovascular diseases only in healthy non-inflammatory patients. From here, we will attempt the detection of other specific biomarkers for CVD.

\subsubsection{Myoglobin (Mb) detection}

In our real-time biophotonic detection of $\mathrm{Mb}$, a concentration of $20 \mathrm{ng} / \mathrm{mL}$ has been flowed. In this time, real human serum (10\%) was used with the PBS-T buffer as a carrier to prevent the conformational changes of the protein, and hence preserve its stability. Also, the introduction of a real matrix such as human serum is a good milestone to checkup the availability of our sensing system in presence of nontargeted elements (e.g., lipids, cells, proteins...). Note that control tests for human real serum were done previously in microarray configuration with no interferences to the bioreceptors. Finally, specific myoglobin antibody was flowed for signal amplification. Results are shown in figure 4.11. 


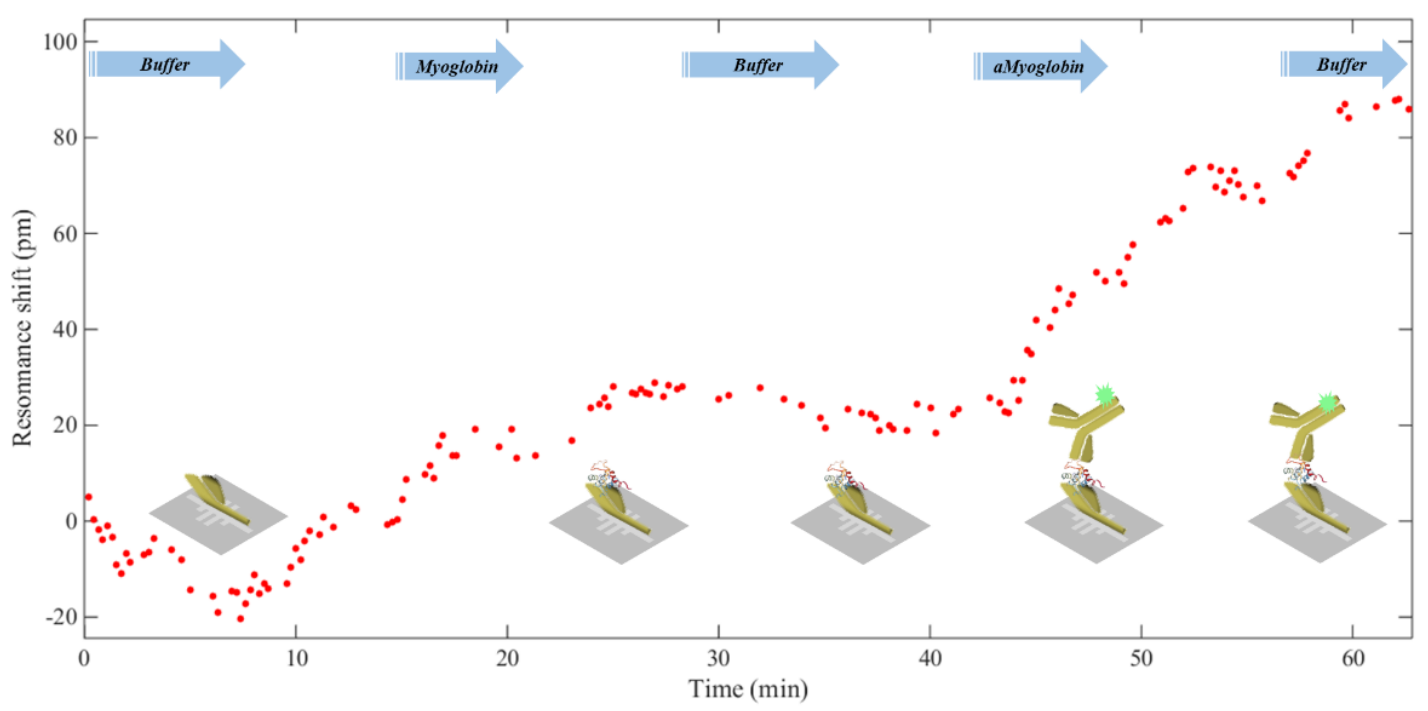

Figure 4.11. $20 \mathrm{ng} / \mathrm{mL}$ Myoglobin detection and representative scheme.

First, buffer composed by PBST+ human serum 10\% was flowed during 10 minutes in order to establish the baseline. Then, $20 \mathrm{ng} / \mathrm{mL}$ of $\mathrm{Mb}$ in the same buffer was flowed for $15 \mathrm{~min}$. Afterwards, buffer was flowed again for 15 minutes to eliminate a possible $\mathrm{Mb}$ excess before flowing a fluorophore-labelled detection antibody in the same buffer for 15 minutes as well. Finally, 10 minutes of buffer was flowed for the fluorophore-labelled $\mathrm{Mb}$ detection antibody excess elimination. A fluorescence test was successfully carried out to double check the detection intensity and homogeneity.

Myoglobin direct detection gave a PBG shift of about $20 \mathrm{pm}$. This shift is smaller comparing to $\mathrm{CRP}$, because from a side, the size and shape of $\mathrm{Mb}$ are considerably smaller than CRP, and from the other side, the concentration flowed is significantly lower $(<0.1 \mathrm{x})$ than for CRP. From here, the need of the Mb detection antibody flow with the aim of enhancing and amplify the sensing signal. Since its size and shape are bigger, $\mathrm{Mb}$ detection antibody provokes a shift of $70 \mathrm{pm}$, thus enhancing the total detection shift from $20 \mathrm{pm}$ to approximately $90 \mathrm{pm}$. 


\subsection{3. cTnI and cTnT detection}

As previously introduced in chapter 1 , clinical studies backed up by bibliographical works (Collinson et al., 2001; Ridker, 1999; Sagarad et al., 2012) have shown that cTnI levels > $10 \mathrm{ng} / \mathrm{L}$ is a CVD high risk biomarker level, being $1 \mathrm{ng} / \mathrm{L}$ a normal cTnI level in bloodstream. Within the PHOCNOSIS project, besides the working on the photonic biodetection, several partners are involved in other aspects, like the electronics, integration and micro/-nanofluidics. The last one has a special interest, since it targets the development, fabrication and testing of a microfluidic cell which is able to concentrate and filter the real human sample. Briefly, it consists in a PDMS cell with several chambers to fill with the buffers and the target sample. By applying an electrical field, the components of the sample are separated by charge allowing the increase of the concentration of sample components in different locations of the microfluidic channel. With this achievement, a protein concentration may increase about x1000. From here, the cTnI and/or cTnT concentrations we should detect using the photonic sensors are in the level of 1 $\mathrm{ng} / \mathrm{mL}$ and $10 \mathrm{ng} / \mathrm{mL}$, respectively, which is the range of our photonic biosensing assays.

As previously done in the rest of biosensing experiments, we apply the same chemical and biochemical functionalization scheme except that the half antibody immobilized on the surface is now specific for cTnI. $100 \mathrm{ng} / \mathrm{mL}$ of cTnI were flowed over the PBG biosensing surface. Note that cTnI is again flowed using a buffer composed by $10 \%$ real human serum in PBST as for Mb biodetection case, to prevent the conformational changes and structural damage of the cTnI protein. Results are depicted in figure 4.12. 


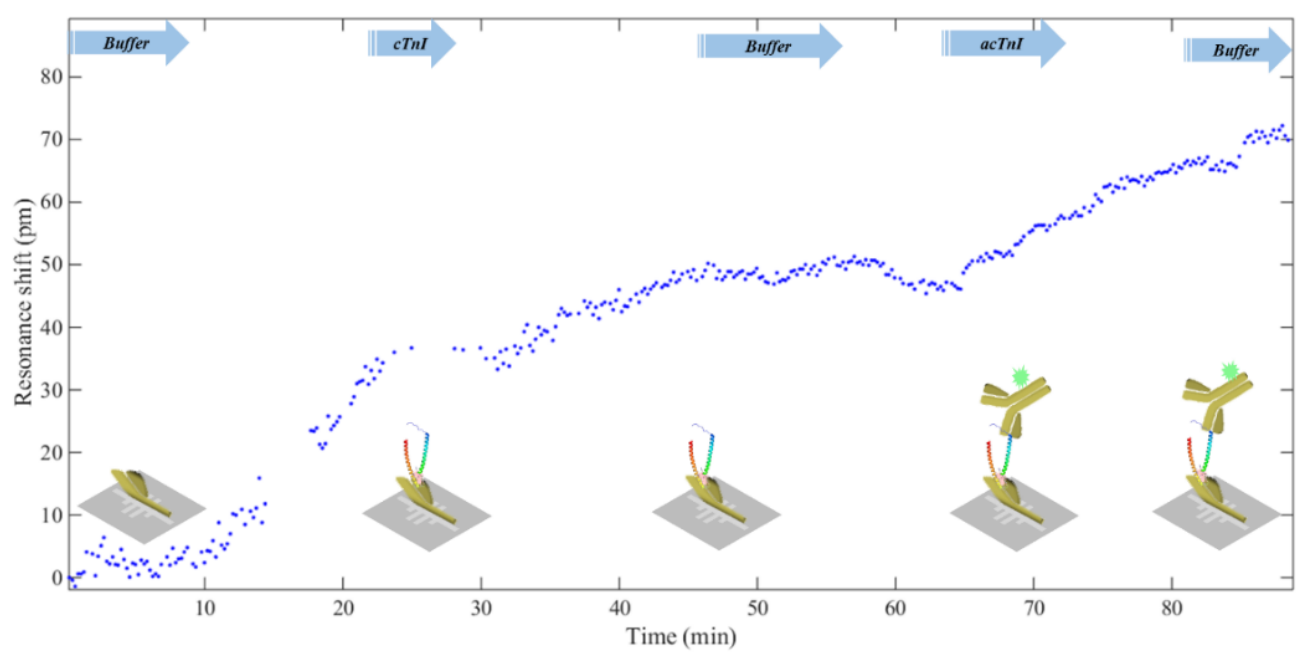

Figure 4.12. $100 \mathrm{ng} / \mathrm{mL} \mathrm{cTnI}$ detection and representative scheme.

Here, we start flowing PBST + human serum 10\% during 10 minutes in order to establish the baseline. Then, $100 \mathrm{ng} / \mathrm{mL}$ of cTnI in the same buffer was flowed for $30 \mathrm{~min}$. Afterwards, the buffer with human serum was flowed again for 20 minutes to eliminate cTnI excess before flowing a fluorophore-labelled detection antibody in the same buffer for 20 minutes as well. Finally, 10 minutes of the buffer was flowed for the cTnI detection antibody excess elimination. A fluorescence test was carried out successfully to double check the achieved detection.

$100 \mathrm{ng} / \mathrm{mL}$ of cTnI direct detection gave a resonance shift of about $50 \mathrm{pm}$. This shift is higher compared to that obtained for $\mathrm{Mb}$, because the size, shape and the concentration of cTnI are also higher than Mb. To keep the same strategy, cTnI detection antibody is flowed with the aim of enhancing and amplifying the sensing signal. Since its size is higher, anticTnI triggers a shift of $25 \mathrm{pm}$, providing a total detection shift for $\mathrm{cTnI} 100 \mathrm{ng} / \mathrm{mL}$ of $75 \mathrm{pm}$. Here, the issue is that the fluorophorelabelled cTnI detection antibody should have provoked a higher PBG shift than the cTnI protein itself (see figure 4.11).

For the next experiment we shift to another CVD biomarker which is the cTnT. Here, $10 \mathrm{ng} / \mathrm{mL}$ of cTnT protein detection assay was performed following the same protocol as the previous experiment. As it can be observed in figure 4.13, no shift was observed for the direct detection of $10 \mathrm{ng} / \mathrm{mL}$ of cTnT, mainly determined by 
the low concentration of the target. However, a shift of around $35 \mathrm{pm}$ was observed when the secondary antibody was flowed.

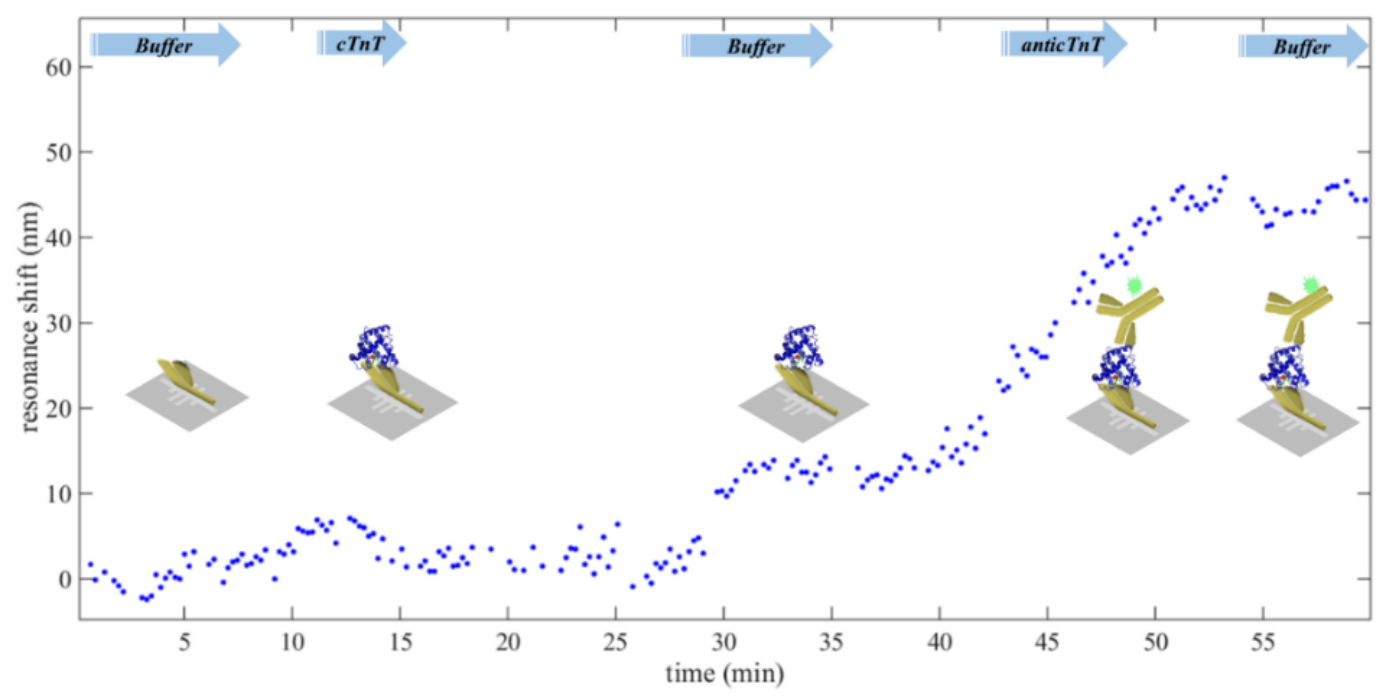

Figure 4.13. $10 \mathrm{ng} / \mathrm{mL}$ cTnT detection and representative scheme.

We also tried to detect a lower concentration of cTnT $(1 \mathrm{ng} / \mathrm{mL})$, but it was unsuccessful. Therefore, in order to deal with this issue, we tried another biofunctionalization protocol that may overcome these limitations and enables us to achieve the required detection limit of $1 \mathrm{ng} / \mathrm{mL}$.

\subsection{Protein $G$ based biofunctionalization for $1 \mathrm{ng} / \mathrm{mL}$ cTnT detection}

We have tested an alternative biofunctionalization approach in order to assess if it was possible to reduce the detection limit of our biosensing experiments. Here, we perform another kind of biofunctionalization consisting on direct physical adsorption. Briefly, this methodology is based on the adsorption of a protein (mostly protein A and/or $\mathrm{G}$ ) followed by the biofunctionalization with the whole antibody and the blocking of the non-bounded sites of the adsorbed protein before the biodetection assay (Caroselli et al., 2018). The approach presented here might be an efficient alternative to the previously used techniques, as it allows to overcome their drawbacks because it enables the presence of double epitopes 
number than for half antibody. Moreover, the proper antibody orientation provided by the presence of the protein A or G layers is translated into a better sensitivity of the photonic structure. Additionally, the whole biofunctionalization process can be directly applied in-flow, thus allowing to flow all the reagents of the whole experiment (from the biofunctionalization to the detection) avoiding dispersed functionalization steps with their respective error margins.

Within this context, the PBG sensing structures biofunctionalization is based on the physical adsorption immobilization of the protein $G$ as an intermediate layer to immobilize the antibodies. So, the antibodies can be anchored to the sensing surface by binding to protein $\mathrm{G}$, which is a microbial surface protein derived from streptococcus cell. Protein $\mathrm{G}$ contains four binding sites specific to the common Fc region of the antibodies. Thus, it specifically binds with Fc regions of the antibodies while the Fab regions stays available, exposed and neatly oriented towards the sample solution including the target protein to be detected. Furthermore, the regeneration is easier in contrast to covalently linked antibody to the surface, since it is possible to break this binding by using the chaotropic agents (glycine, $\mathrm{NaOH}$, $\mathrm{NaCl}, \ldots)$ in order to biofunctionalize again with a new batch of antibodies of the same kind and repeat the same experiment or either the use of another type of antibodies and carry out a different sensing assay. The complete protocol is schematized in figure 4.14.

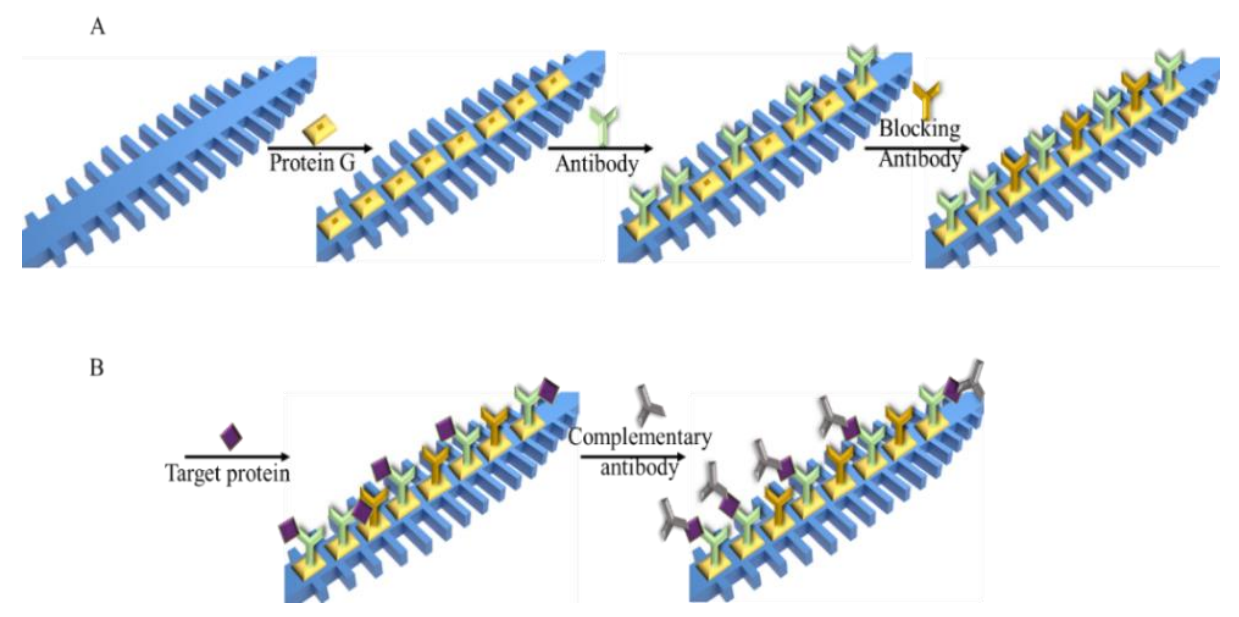

Figure 4.14. (A) Schematic representation of the biofunctionalization protocol and (B) schematic representation of the detection. 
First, we start flowing protein G over the PBG sensing structures with the aim of the creation of the protein $G$ intermediate layer by physical adsorption, which will ensure us a high surface coverage and oriented immobilization of the antibody. First, PBS was flowed during 10 minutes in order to stablish a baseline. Then, protein $\mathrm{G}$ in PBS with a concentration of $20 \mu \mathrm{g} / \mathrm{mL}$ at $10 \mu \mathrm{L} / \mathrm{min}$ flow rate was flowed during 20 minutes before we flowed again the PBS to eliminate the excess of protein G. Note that we do not use PBST in this case, since the surfactant does not help promote the physical surface adsorption. Here, 100 pm of PBG shift was observed due to the physical adsorption of the protein $G$ over the sensing surface. Results are presented in figure 4.15 .

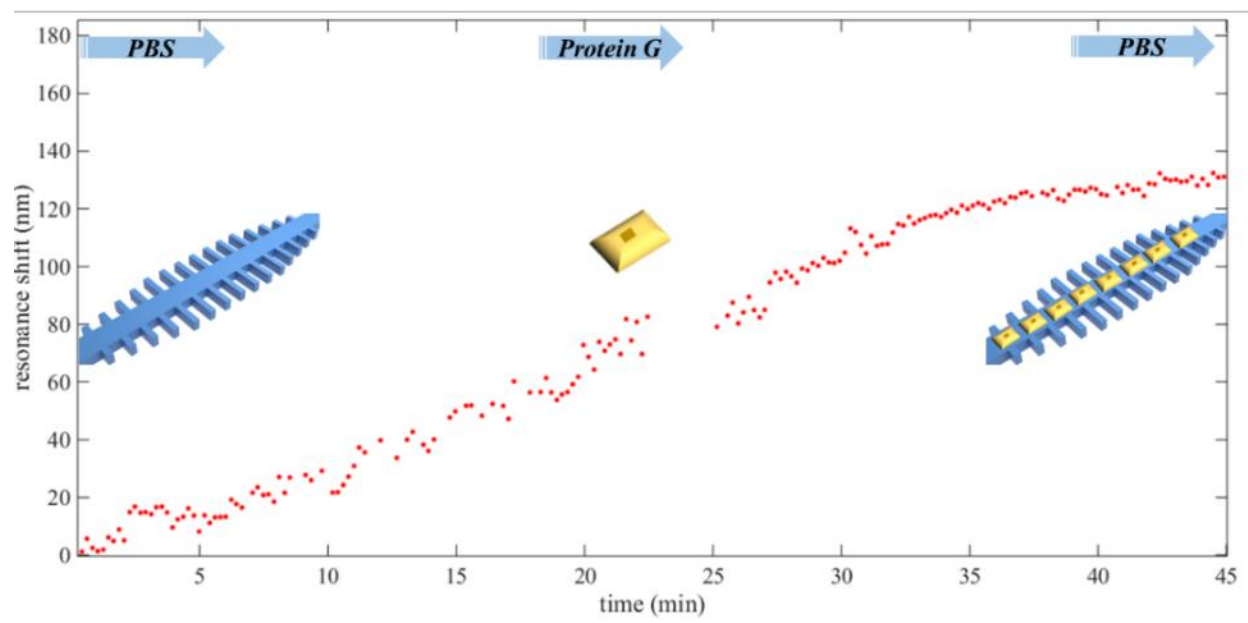

Figure 4.15. Protein $\mathrm{G}$ adsorption over the sensing surface and representative scheme.

After the creation of the intermediate layer of protein G, cTnT antibody was flowed at a concentration of $20 \mu \mathrm{g} / \mathrm{ml}$ in PBST over the sensing surface with the adsorbed protein $\mathrm{G}$ layer. Here, the tween 20 surfactant is used to avoid the adsorption of the cTnT antibody on the sensor surface, since that physical adsorption of the antibody might provide non-oriented antibodies, thus decreasing the sensing capability. Furthermore, the interaction of the antibody with the protein $G$ is a binding interaction between the Fc region of the cTnT antibody and the protein $\mathrm{G}$ binding sites. PBST buffer was flowed for 10 minutes to settle a baseline before flowing 20 $\mu \mathrm{g} / \mathrm{mL}$ of rabbit anticTnT for 25 minutes to bind the antibodies to the Fc protein $\mathrm{G}$ binding sites. Then, PBST is flowed once again for 10 minutes to eliminate the 
excess of the anticTnT. A total shift of $120 \mathrm{pm}$ was achieved, as depicted in figure 4.16 .

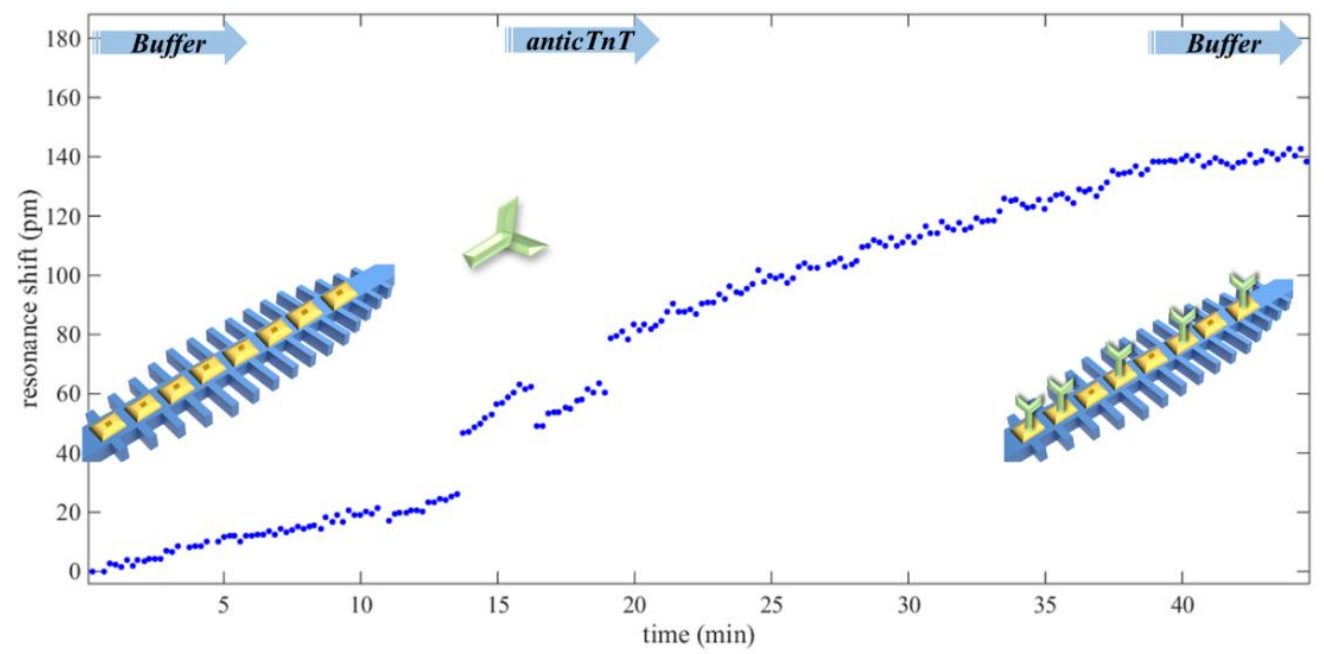

Figure 4.16. anticTnT binding to the protein $\mathrm{G}$ and representative scheme.

The next step consists on the blocking antibody flow. It is used with the aim of blocking the available non-bounded sites of the protein $\mathrm{G}$ by anticTnT. A higher concentration $(200 \mu \mathrm{g} / \mathrm{mL})$ was flowed to ensure the complete blocking of the available protein $\mathrm{G}$ sites. Here, a PBST buffer baseline was stablished for 13 minutes before the flow of the blocking antibody in the same buffer for 15 minutes. Then, the buffer was flowed again to eliminate the antibody excess, from here the signal decrease starting from min 28 . The total net shift was in the order of $280 \mathrm{pm}$. Results are depicted in figure 4.17.

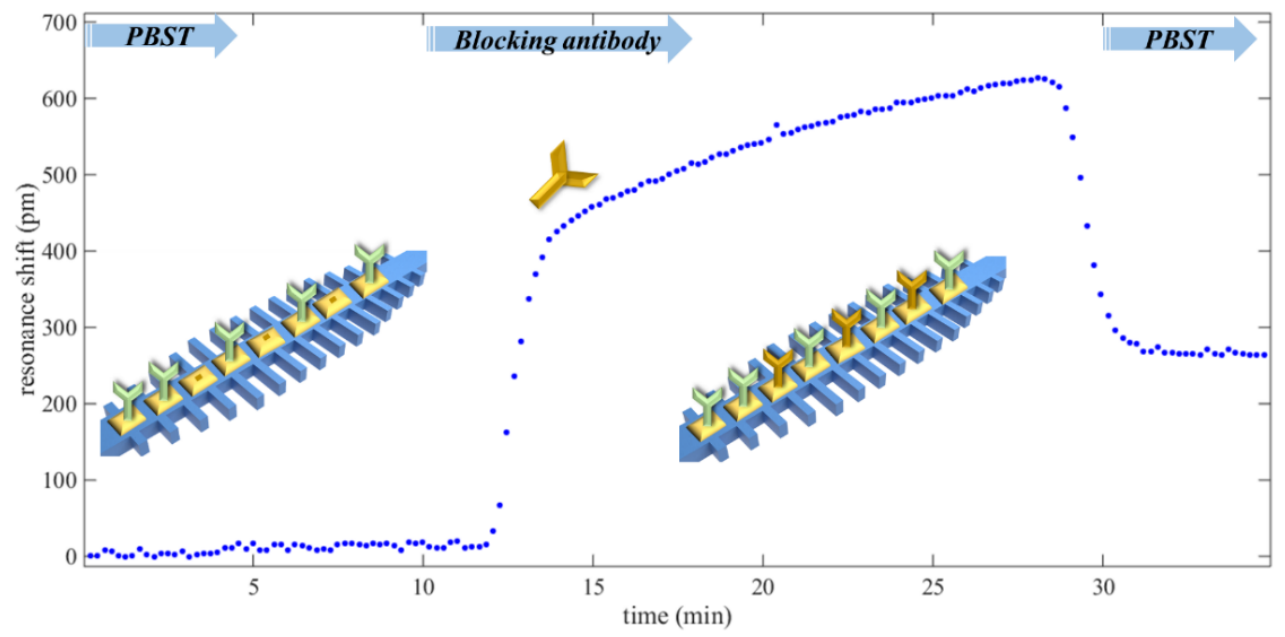

Figure 4.17. Blocking antibody binding to protein $\mathrm{G}$ and representative scheme. 
Once the biofunctionalization process was completed, we proceeded with the detection of $1 \mathrm{ng} / \mathrm{mL}$ of cTnT. As previously performed for other CVD biomarkers, the detection protocol will be based on the flow of the PBST buffer with real human serum at $10 \%$ for 10 min followed by the flow of the target protein cTnT for 15 min. The buffer is flowed again in order to eliminate excess of cTnT and finally anticTnT is flowed for $20 \mathrm{~min}$ in order to amplify the sensing signal. Finally, the buffer was flowed to eliminate the rests of the fluorophore-labelled detection anticTnT antibody. Results are depicted in the figure 4.18 .

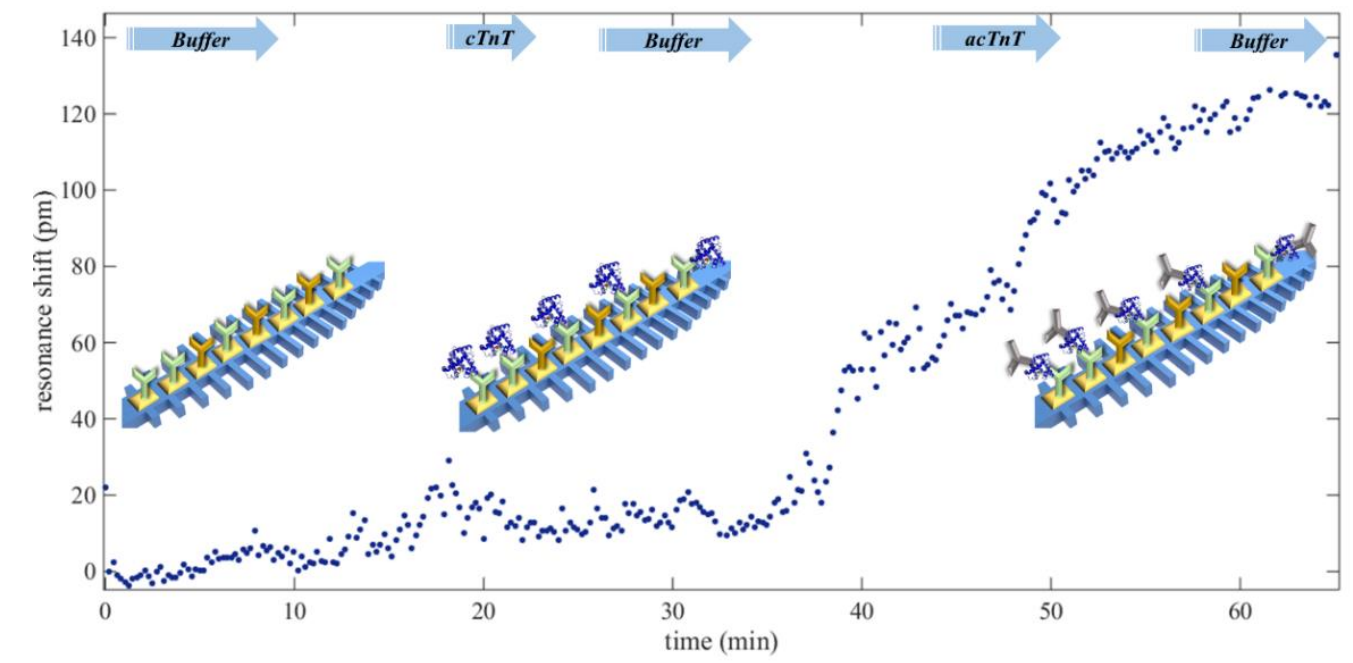

Figure 4.18. $1 \mathrm{ng} / \mathrm{mL} \mathrm{cTnT}$ protein detection and representative scheme.

Here, and when $1 \mathrm{ng} / \mathrm{mL}$ of cTnT achieves the sensing structures, we can observe a slight shift of about $10 \mathrm{pm}$ which is enhanced by $100 \mathrm{pm}$ when the detection antibody is flowed, making the total PBG shift reaching $110 \mathrm{pm}$.

\subsection{Conclusions}

In this chapter, we have developed a photonic biosensing system based on PBG sensing structures for the specific detection of proteins. First, we have performed a study of the evanescent field profile. A strong exponential decay has been observed in the SNOM characterization carried out, thus highlighting the importance of having a biorecognition layer as thin as possible for a high sensitivity detection. Additionally, note that a good agreement has been observed between SNOM 
experimental measurements and FDTD near field behavior simulations, thus making this simulation method appropriate for characterizing evanescent field photonic sensors with a high accuracy.

To fulfill the requirement of having a very thin biorecognition layer, we implemented the TEC biofunctionalization protocol. Then, and by means of BSA detection experiments, we have confirmed that a high sensitivity is obtained. We have also observed that this sensitivity might also be higher by properly tuning the structural dimensions of the PBG sensing structures, as simply an increase of the transversal elements width was translated into a sensitivity increase by approx. $\times 3$. Next, CVD biomarkers such as CRP and Mb were detected. The CRP was detected directly at $250 \mathrm{ng} / \mathrm{mL}$ with a net PBG shift of $65 \mathrm{pm}$ whereas the Mb was detected with $20 \mathrm{pm}$ directly and up to $90 \mathrm{pm}$ by flowing the detection antibody. Then, cTnI at $100 \mathrm{ng} / \mathrm{mL}$ gave a $50 \mathrm{pm}$ PBG shift while a direct detection when the detection antibody increases this detection until $75 \mathrm{pm}$. The decrease of the concentration to $10 \mathrm{ng} / \mathrm{mL}$ with a shift to cTnT was performed for the next experiment. Here, 10 $\mathrm{ng} / \mathrm{mL}$ of cTnT only gave PBG shift due to the flow of the detection antibody with a PBG shift of $35 \mathrm{pm}$. For $1 \mathrm{ng} / \mathrm{mL}$ of cTnT, no PBG shift was observed. So, we have to deal with the issue by changing the functionalization protocol. This biofunctionalization way was based on the protein $\mathrm{G}$ physical adsorption upon the sensing surface and antibody functionalization was used with successful results regarding the decrease of the experimental limit of detection, which demonstrates among others, the functionalization versatility of the PBG sensing structures. In our knowledge, this is the first time that $1 \mathrm{ng} / \mathrm{mL}$ of a cTnT biomarker is detected using a PBG biosensor. Moreover, direct detection of lower normal range concentration of CRP (250 ng/mL) was directly detected without need of the detection antibody. These results are highly important, since they may open a wide range of applications for other biomarkers for diseases diagnostics. 


\subsection{References}

Alonso, R., Jiménez-Meneses, P., García-Rupérez, J., Bañuls, M.-J., Maquieira, Á., 2018. Thiol-ene click chemistry towards easy microarraying of halfantibodies. Chem. Commun. 54, 6144-6147. https://doi.org/10.1039/C8CC01369A

Aul, P., Idker, M.R., Harles, C., Ennekens, H.H., Ulie, J., Uring, E.B., Ader, N., Ifai, R., 2000. C-Reactive protein and other markers of inflammation in the prediction of cardiovascular didease in women.

Bazylewski, P., Ezugwu, S., Fanchini, G., Bazylewski, P., Ezugwu, S., Fanchini, G., 2017. A Review of Three-Dimensional Scanning Near-Field Optical Microscopy (3D-SNOM) and Its Applications in Nanoscale Light Management. Appl. Sci. 7, 973. https://doi.org/10.3390/app7100973

Blake, G., Ridker, P.M., 2001. High sensitivity C-reactive protein for predicting cardiovascular disease: an inflammatory hypothesis. Eur. Heart J. 22, 349352. https://doi.org/10.1053/euhj.2000.2280

Caroselli, R., García Castelló, J., Escorihuela, J., Bañuls, M., Maquieira, Á., GarcíaRupérez, 2018. Experimental Study of the Oriented Immobilization of Antibodies on Photonic Sensing Structures by Using Protein A as an Intermediate Layer. Sensors 18, 1012. https://doi.org/10.3390/s18041012

Collinson, P., Boa, F.G., Gaze, D.C., 2001. Measurement of cardiac troponins, Review Article Ann Clin Biochem.

Díaz-Fernández, F.J., Pinilla-Cienfuegos, E., García-Meca, C., Lechago, S., Griol, A., Martí, J., 2018. Characterisation of on-chip wireless interconnects based on silicon nanoantennas via near-field scanning optical microscopy. IET Optoelectron. 6. https://doi.org/10.1049/iet-opt.2018.5071

Dvořák, P., Édes, Z., Kvapil, M., Šamořil, T., Ligmajer, F., Hrtoň, M., Kalousek, R., Křápek, V., Dub, P., Spousta, J., Varga, P., Šikola, T., 2017. Imaging of near-field interference patterns by aperture-type SNOM - influence of 
illumination wavelength and polarization state. Opt. Express 25, 16560. https://doi.org/10.1364/OE.25.016560

Eisenberg, M.S., Chen, H.J., Warshofsky, M.K., Sciacca, R.R., Wasserman, H.S., Schwartz, A., Rabbani, L.E., 2000. Elevated Levels of Plasma C-Reactive Protein Are Associated With Decreased Graft Survival in Cardiac Transplant Recipients.

Espinosa-Soria, A., Pinilla-Cienfuegos, E., Díaz-Fernández, F.J., Griol, A., Martí, J., Martínez, A., 2018. Coherent Control of a Plasmonic Nanoantenna Integrated on a Silicon Chip. ACS Photonics 5, 2712-2717. https://doi.org/10.1021/acsphotonics.8b00447

Estevez, M.C., Alvarez, M., Lechuga, L.M., 2012. Integrated optical devices for lab-on-a-chip biosensing applications. Laser Photon. Rev. 6, 463-487. https://doi.org/10.1002/lpor.201100025

Fan, X., White, I.M., Shopova, S.I., Zhu, H., Suter, J.D., Sun, Y., 2008. Sensitive optical biosensors for unlabeled targets: A review. Anal. Chim. Acta 620, 8 26. https://doi.org/10.1016/J.ACA.2008.05.022

García-Rupérez, J., Toccafondo, V., Bañuls, M.J., Castelló, J.G., Griol, A., PeransiLlopis, S., Maquieira, Á., 2010. Label-free antibody detection using band edge fringes in SOI planar photonic crystal waveguides in the slow-light regime. Opt. Express 18, 24276. https://doi.org/10.1364/OE.18.024276

Garcia, J., Sanchis, P., Martinez, A., Marti, J., 2008. 1D periodic structures for slow-wave induced non-linearity enhancement. Opt. Express 16, 3146. https://doi.org/10.1364/OE.16.003146

Johnson, B.N., Mutharasan, R., 2012. Biosensing using dynamic-mode cantilever sensors: A review. Biosens. Bioelectron. 32, 1-18. https://doi.org/10.1016/J.BIOS.2011.10.054

Johnson, S.G., Joannopoulos, J.D. (John D.., 2002. Photonic crystals : the road from theory to practice. Kluwer Academic Publishers. 
Kuchinsky, S., Golyatin, V.Y., Kutikov, A.Y., Pearsall, T.P., Nedeljkovic, D., 2002. Coupling between photonic crystal waveguides Text-2 View project Thin heterostructure layer growth View project Coupling Between Photonic Crystal Waveguides. Artic. IEEE J. Quantum Electron. 38, 1349. https://doi.org/10.1109/JQE.2002.802954.

Lliott, E., Ntman, M.A., Anasijevic, I.J.T., Ark, M., Chactman, S., Abe, A.H.M.C.C., Annon, H.P.C., Ischer, E.A.F., Ung, N.Y.F., Hristopher, C., Hompson, T., Onald, D., Ybenga, W., Ugene, E., Raunwald, B., 1996. Cardiac-specific troponin I levels to predict the risk of mortality in patients with acute coronary syndromes. Background In patients with acute coronary syn, The New England Journal of Medicine.

Luchansky, M.S., Washburn, A.L., McClellan, M.S., Bailey, R.C., 2011. Sensitive on-chip detection of a protein biomarker in human serum and plasma over an extended dynamic range using silicon photonic microring resonators and submicron beads. Lab Chip 11, 2042. https://doi.org/10.1039/c1lc20231f

McManus, J.L., 1993. Cardiac troponin I: A marker with high specificity for cardiac injury. Ann. Emerg. Med. 22, 1930-1931. https://doi.org/10.1016/S01960644(05)80440-8

Meyer, V., Kober, C., Niessner, R., Seidel, M., 2015. Regeneration of Recombinant Antigen Microarrays for the Automated Monitoring of Antibodies against Zoonotic Pathogens in Swine Sera. Sensors 15, 2614-2628. https://doi.org/10.3390/s150202614

Myoglobin - Serum | Medical Tests | UCSF Medical Center, n.d. URL https://www.ucsfhealth.org/tests/003663.html (accessed 3.26.19).

Namiki, T., 1999. A new FDTD algorithm based on alternating-direction implicit method. IEEE Trans. Microw. Theory Tech. 47, 2003-2007. https://doi.org/10.1109/22.795075

Phaner-Goutorbe, M., Dugas, V., Chevolot, Y., Souteyrand, E., 2011. Silanization of silica and glass slides for DNA microarrays by impregnation and gas phase 
protocols: A comparative study. Mater. Sci. Eng. C 31, 384-390. https://doi.org/10.1016/J.MSEC.2010.10.016

Rheims, J., Köser, J., Wriedt, T., 1997. Refractive-index measurements in the nearIR using an Abbe refractometer. Meas. Sci. Technol. 8, 601-605. https://doi.org/10.1088/0957-0233/8/6/003

Ridker, P.M., 1999. Evaluating Novel Cardiovascular Risk Factors: Can We Better Predict Heart Attacks? Ann. Intern. Med. 130, 933. https://doi.org/10.7326/0003-4819-130-11-199906010-00018

Ruiz-Tórtola, Á., Prats-Quílez, F., González-Lucas, D., Bañuls, M.-J., Maquieira, Á., Wheeler, G., Dalmay, T., Griol, A., Hurtado, J., García-Rupérez, J., 2018. High sensitivity and label-free oligonucleotides detection using photonic bandgap sensing structures biofunctionalized with molecular beacon probes. Biomed. Opt. Express 9, 1717. https://doi.org/10.1364/BOE.9.001717

Sagarad, S. V, Singh Thakur, B., Reddy, Balasubramanya, S.S., Joshi, M., Kerure, S.B., 2012. Elevated Cardiac Troponin (cTnI) Levels Correlate with the Clinical and Echocardio-graphic Evidences of Severe Myocarditis in Scorpion Sting Envenomation. J. Clin. Diagnostic Res. 6, 1369-1371. https://doi.org/10.7860/JCDR/2012/4760.2361

Schuetz, P., Affolter, B., Hunziker, S., Winterhalder, C., Fischer, M., Balestra, G.M., Hunziker, P., Marsch, S., 2010. Serum procalcitonin, C-reactive protein and white blood cell levels following hypothermia after cardiac arrest: a retrospective cohort study. Eur. J. Clin. Invest. 40, 376-381. https://doi.org/10.1111/j.1365-2362.2010.02259.x

Skivesen, N., Têtu, A., Kristensen, M., Kjems, J., Frandsen, L.H., Borel, P.I., 2007. Photonic-crystal waveguide biosensor.

Vaidya, H.C., 1992. Myoglobin. Lab. Med. 23, 306-310. https://doi.org/10.1093/labmed/23.5.306 
Wang, Z., Jin, G., 2003. Feasibility of protein A for the oriented immobilization of immunoglobulin on silicon surface for a biosensor with imaging ellipsometry. J. Biochem. Biophys. Methods 57, 203-11.

Washburn, A.L., Bailey, R.C., 2011. Photonics-on-a-chip: recent advances in integrated waveguides as enabling detection elements for real-world, lab-ona-chip biosensing applications. Analyst 136, 227-236. https://doi.org/10.1039/C0AN00449A

Wittenberg, J.B., Wittenberg, B.A., 2003. Myoglobin function reassessed. J. Exp. Biol. 206, 2011-20. https://doi.org/10.1242/JEB.00243

Wu, J., Dong, M., Santos, S., Rigatto, C., Liu, Y., Lin, F., Wu, J., Dong, M., Santos, S., Rigatto, C., Liu, Y., Lin, F., 2017. Lab-on-a-Chip Platforms for Detection of Cardiovascular Disease and Cancer Biomarkers. Sensors 17, 2934. https://doi.org/10.3390/s17122934

Yablonovitch, E., 1993. Photonic band-gap structures. J. Opt. Soc. Am. B 10, 283. https://doi.org/10.1364/JOSAB.10.000283. 


\section{Chapter 5}

\section{Conclusions and future}

\section{steps}

\subsection{Conclusions}

In this $\mathrm{PhD}$ Thesis, we have started studying theoretically and computationally the binding of the cardiac troponin I CVD biomarker with its antibody. First, it was necessary to determine the binding sites of the antibody and the antigen as well. This step was performed using FTSite and FTMap with the aim of determining these binding regions or hot spots. Then, a computational docking was made using pyDock and FTDock to determine the availability, strength of the binding and number of bonds between the cTnI and the anticTnI. This work was complemented by a computational selectivity study by introducing the skeletal troponin I (sTnI) as principal interferent. The obtained results show the higher specificity of the antic $\mathrm{TnI}$ to the $\mathrm{cTnI}$ rather than $\mathrm{sTnI}$.

Next, a real-time biofunctionalization study was performed over the PBG sensing structures, which were nanofabricated on a chip including 16 structures varying the parameter $\mathrm{w}_{\mathrm{i}}(80,100,120$ and $140 \mathrm{~nm})$ allowing to have PBGs in different wavelengths. This chip was integrated in an optofluidic set-up to track in real-time the PBG shift during the UV-light assisted biofunctionalization process. On the other side, BSA antibodies were divided using the TCEP protocol in order to obtain two SH-terminated moieties. This part was done in collaboration with UPV-SYM. The chip was silanized using TEVS and integrated with the microfluidic cell and aligned in the photonic set-up. Since the reaction of thiol-ene coupling is UV-light dependent, a $254 \mathrm{~nm}$ wavelength lamp was switched to photo-catalyze the TEC 
reaction. The PBG edges were shifted towards different wavelengths due to the different sensitivities announcing the accomplishment of the half antibodies biofunctionalization to the PBG sensors.

Also, Scanning Near-field Optical Microscope characterization was employed to experimentally study the evanescent field behavior. This study allowed us to better understand the evanescent field behavior and substantiate the availability of our biofunctionalization protocol to perform the sensing study with the optimal sensitivity. In fact, as thinnest is the biolayer, the interaction between the target analyte and the evanescent field is maximal and hence, the shift is higher for very low concentrations of the target analyte.

Then, biodetection experiments were carried out, but changing the immobilization of the antibodies from an in-flow format to spotting in order to improve the surface coverage, volumes and time. The first experiments were carried out using the model system BSA-antiBSA at $1 \mu \mathrm{g} / \mathrm{mL}$ with successful detection results. Then, we tried with different CVD biomarkers systems some of them at clinical levels (CRP and $\mathrm{Mb})$ achieving successful results.

In order to further reduce the detection limit of our photonic biosensor, another biofunctionalization system was used and tested for cTnT detection. It consists on a physical adsorption of protein $\mathrm{G}$, followed by binding of the antibodies at their Fc regions to the protein $\mathrm{G}$ layer, followed by the blocking of the non-bounded protein $\mathrm{G}$ sites using a blocking antibody. $1 \mathrm{ng} / \mathrm{mL}$ of cTnT detection was achieved satisfying the work objective. Here, the detection was done by means of the flow of the complementary antibody for the cardiac Troponin $\mathrm{T}$.

As general conclusion, we can say that the targeted detections were achieved, but several issues were observed. This may be due to the following reasons:

The nanofabrication gave some dimensions mismatches in $\mathrm{w}_{\mathrm{i}}$ (2 to $12 \mathrm{~nm}$ ) and hence the PBG edges of the same parameters were localized in different wavelengths, thus varying the sensitivity. This issue was found in same structures at the chip and in different ones as well. 
The chemical functionalization was reproducible but not at $100 \%$. For example, the Water Contact Angle (WCA) varies between $72^{\circ}$ and $85^{\circ}$, what might be due to differences of the TEVS batches, volumes, temperature variations and/or humidity.

The half antibodies also show sometimes a low binding capability, what might indicate that there are also some variations in the TCEP reduction protocol used to obtain the hIgGs and either in their storage process.

The microfluidic system also presents some limitations, since sometimes bubbles, obstructions, changes in the flow rate and holder chip movements when changing the tubes in the experiments were observed in the signals, what influences the performance of the biosensing experiments.

\subsection{Future steps}

From the obtained results, many optimizations can be performed to overcome all the limitations found at this stage. For example, the use of other kind of receptors such as nanobodies, gold nanoparticles intermediates or either different photonic structures being easier to fabricate in our cleanroom facilities techniques in order to have more reproducible spectra and sensitivities and jump to the multiplexed detection milestone. However, the developed PBG sensing structures are hopefully available for micrograms per milliliter concentrations proteins detection. Also, as a semi-quantitative sensing system immunoassay tests, which can have several applications in a wide range of fields such as food industry, defense and other several healthcare applications. 


\section{Author publications}

\section{Journal publications}

[1]. Sabek, J., Martínez-Pérez, P., García-Rupérez, J., 2019. Computational binding study of cardiac troponin I antibody towards cardiac versus skeletal troponin I. Comput. Biol. Chem. 80, 147-151. https://doi.org/10.1016/j.compbiolchem.2019.04.002.

[2]. Sabek, J., Torrijos-Morán, L., Griol, A., Díaz Betancor, Z., Bañuls Polo, M.-J., Maquieira, Á., García-Rupérez, J., 2018. Real Time Monitoring of a UV Light-Assisted Biofunctionalization Protocol Using a Nanophotonic Biosensor. Biosensors 9, 6. https://doi.org/10.3390/bios9010006.

[3]. Bañuls, M.-J., González-Martínez, M.Á., Sabek, J., García-Rupérez, J., Maquieira, Á., 2019. Thiol-click photochemistry for surface functionalization applied to optical biosensing. Anal. Chim. Acta 1060, 103-113. https://doi.org/10.1016/j.aca.2019.01.055.

[4]. Sabek, J., Díaz-Fernández, F. J., Torrijos-Morán, L., Díaz-Betancor, Z., Maquieira, Á., Bañuls, M. J., Pinilla Cienfuegos E. \& GarcíaRupérez, J. 2019. Experimental study of an evanescent-field biosensor based on 1D photonic bandgap structures. Beilstein Journal of Nanotechnology, 10(1), 967-974. doi:10.3762/bjnano.10.97. 


\section{Conference publications}

[1]. Sabek, J., Martínez-Pérez, P., \& García-Rupérez, J. (2018). Affinity and Selectivity of Cardiac Versus Skeletal Troponin I Towards Cardiac Troponin I Antibody: A Computational Study. In Multidisciplinary Digital Publishing Institute Proceedings (Vol. 4, No. 1, p. 41).

[2]. Sabek, J., Torrijos-Morán, L., Díaz-Betancor, Z., Bañuls-Polo, M. J., Maquieira-Catalá, Á., \& García-Rupérez, J. (2018). Live Tracking Biofunctionalization and Label-Free Protein Detection Performed by a Nanophotonic Biosensor. In Multidisciplinary Digital Publishing Institute Proceedings (Vol. 4, No. 1, p. 15).

[3]. Sabek, J., Martínez-Pérez, P., \& García-Rupérez, J. (2018) Theoretical and computational study of the affinity of cardiac versus skeletal troponin I towards cardiac troponin I antibody, XII International Workshop on Sensors and Molecular Recognition (IWOSMOR 2018) ISBN:978-84-09-05881-5.

[4]. Sabek, J., Torrijos-Morán, L., Díaz-Betancor, Z., Bañuls-Polo, M. J., Maquieira-Catalá, Á., \& García-Rupérez, J. (2018) In situ functionalization and label free protein detection using a nanophotonic biosensor, XII International Workshop on Sensors and Molecular Recognition (IWOSMOR 2018) ISBN:978-84-09-05881-5. 